University of Louisville

ThinkIR: The University of Louisville's Institutional Repository

Electronic Theses and Dissertations

$5-2021$

\title{
I'm still valid: an explanatory sequential mixed-methods study of part-time phd students' motivation and satisfaction.
}

Heather A Turner

University of Louisville

Follow this and additional works at: https://ir.library.louisville.edu/etd

Part of the Higher Education Commons

\section{Recommended Citation}

Turner, Heather A, "I'm still valid: an explanatory sequential mixed-methods study of part-time phd students' motivation and satisfaction." (2021). Electronic Theses and Dissertations. Paper 3592.

https://doi.org/10.18297/etd/3592

This Doctoral Dissertation is brought to you for free and open access by ThinkIR: The University of Louisville's Institutional Repository. It has been accepted for inclusion in Electronic Theses and Dissertations by an authorized administrator of ThinkIR: The University of Louisville's Institutional Repository. This title appears here courtesy of the author, who has retained all other copyrights. For more information, please contact thinkir@louisville.edu. 
I'M STILL VALID:

AN EXPLANATORY SEQUENTIAL MIXED-METHODS STUDY OF PART-TIME PHD STUDENTS' MOTIVATION AND SATISFACTION

\author{
By \\ Heather A. Turner \\ B.A., Temple University, 2012 \\ M.A., University of Delaware, 2014 \\ M.A., University of Louisville, 2017 \\ A Dissertation \\ Submitted to the Faculty of the \\ College of Education and Human Development of the University of Louisville \\ In Partial Fulfillment of the Requirements \\ For the Degree of \\ Doctor of Philosophy \\ In Educational Leadership and Organizational Development \\ Department of Educational Leadership, Evaluation, and Organizational Development \\ University of Louisville \\ Louisville, Kentucky
}

May 2021 


\section{Copyright 2021}

by Heather A. Turner

All rights reserved 

I'M STILL VALID: AN EXPLANATORY SEQUENTIAL MIXED-METHODS STUDY OF PART-TIME PHD STUDENTS' MOTIVATION AND SATISFACTION

By

Heather A. Turner

A Dissertation Approved on

February 4, 2021

By the following Dissertation Committee:

Megan Pifer, Dissertation Chair

Jason Immekus

Jessica Buckley

Amy Hirschy 


\section{DEDICATION}

This dissertation is dedicated to all other part-time $\mathrm{PhD}$ students. 


\section{ACKNOWLEDGEMENTS}

First and foremost, I want to acknowledge the invaluable guidance, support, and friendship of my advisor and mentor, Dr. Meghan Pifer. Interviewing other doctoral students made me acutely aware of how incredibly fortunate I was to have an advisor who was always available, attentive, and understanding. I truly would not have been able to accomplish this project without her help and insight. I would also like to acknowledge the contributions and guidance of my committee members, Dr. Jason Immekus, Dr. Jessica Buckley, and Dr. Amy Hirschy, as well as the previous support of my masters' advisors, Dr. Jeffrey Sun and Dr. Melissa Ianetta. I am forever grateful for the time and effort you all have devoted to my development. Finally, I would like to acknowledge the unwavering support, encouragement, and love from my family, Joe, Anya, and Greta. 


\begin{abstract}
I'M STILL VALID: AN EXPLANATORY SEQUENTIAL MIXED-METHODS

STUDY OF PART-TIME PHD STUDENTS' MOTVATION AND SATISFACTION

Heather A. Turner
\end{abstract}

March 4, 2021

Doctoral attrition rates are consistently documented at approximately $50 \%$ in the United States, and attrition rates are typically higher for all students who pursue degrees on a part-time basis, regardless of degree level. Yet an increasing number of students are deciding to pursue research doctorates on a part-time basis. This growth in the part-time PhD student population requires an understanding of the factors that affect their persistence. I investigated part-time $\mathrm{PhD}$ student persistence through an explanatory sequential mixed methods approach to understanding the relationship between motivation and satisfaction through the lens of Self-Determination Theory. Findings suggest that part-time $\mathrm{PhD}$ students are motivated by knowledge advancement and relationships with others, yet they may be lacking in access to community in ways that contribute to negative outcomes physically, psychologically, and intellectually. Like many doctoral students, they are demotivated when they feel isolated, exhausted, and overwhelmed; part-time enrollment exacerbates these feelings. Motivation to enroll part-time is largely dictated by family circumstances (e.g., financial need, caregiving responsibilities) and a desire for career advancement. Like motivation, satisfaction is primarily derived through relationships with others, feeling supported, and personal and professional growth. 
However, a lack of community access inhibits part-time student socialization, and may lead to untested assumptions regarding faculty roles and the purpose of research doctoral education. Additionally, part-time students appear reticent to report feelings of overall dissatisfaction, despite indicating feeling dissatisfied with many components of the doctoral experience. Implications for research and practice are discussed. 


\section{TABLE OF CONTENTS}

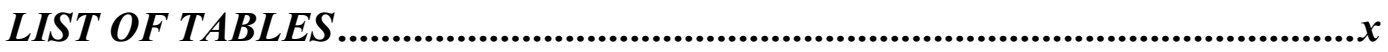

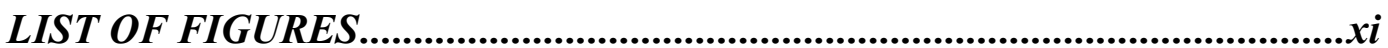

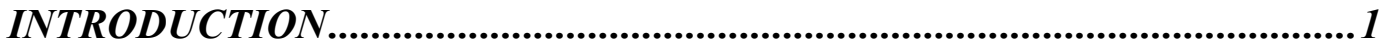

Nontraditional Doctoral Students ..............................................................2

Study Purpose

The Problem .................................................................................................5

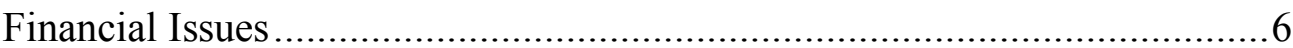

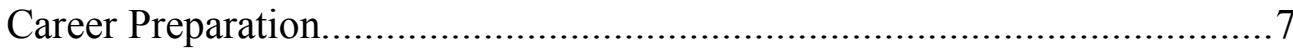

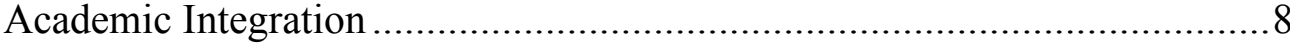

Research Questions ............................................................................10

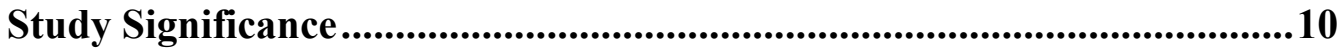

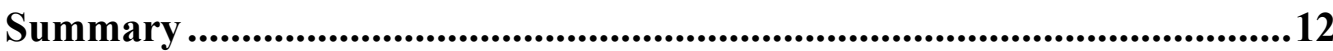

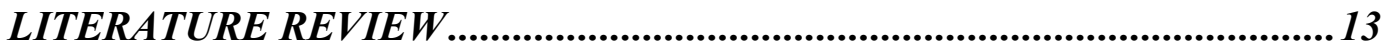

Doctoral Education .................................................................................. 14

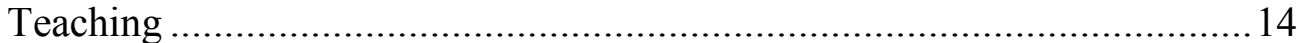

Doctoral Program Design ...................................................................... 15

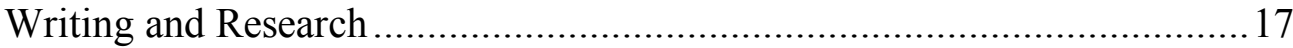

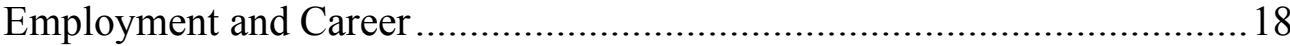

Student-Supervisor Relationship ..................................................... 19

The Doctoral Student Experience...............................................................20

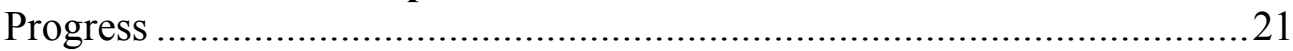

Student Support.........................................................................22

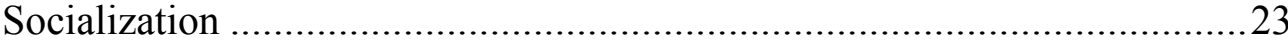

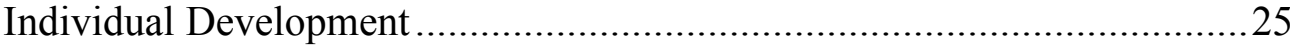

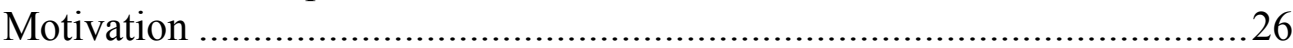

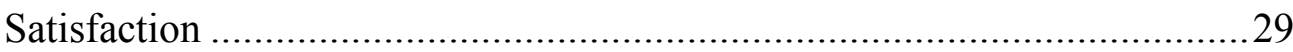

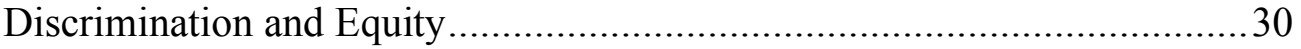


Theoretical Framework .............................................................................32

Summary .............................................................................................35

METHODOLOGY AND RESEARCH DESIGN ......................................37

Study Design........................................................................................38

Relationship between Quantitative and Qualitative Data...........................38

Sample ........................................................................................................41

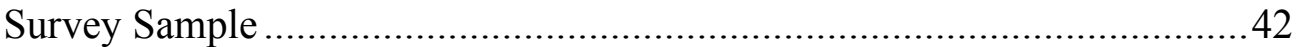

Focus Group Sample...................................................................... 44

Instrumentation ...............................................................................45

Quantitative Instrument..............................................................45

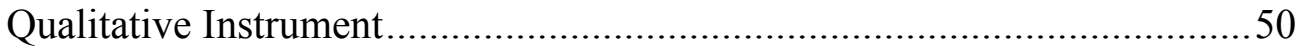

Data Collection.................................................................................51

Quantitative Data Collection ..............................................................51

Qualitative Data Collection ...............................................................5

Data Analysis................................................................................53

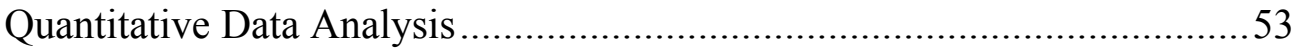

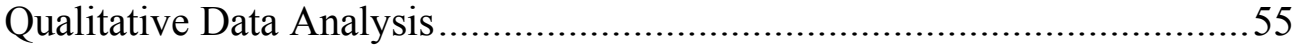

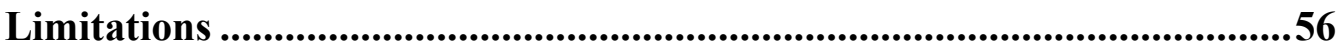

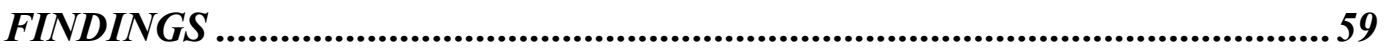

Quantitative Findings ..................................................................60

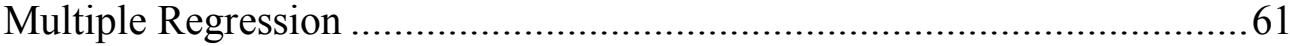

Qualitative Findings....................................................................64

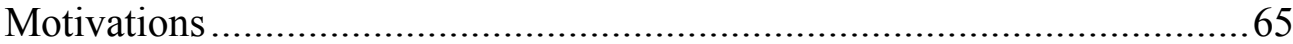

Autonomous Motivations .......................................................................65

Controlled Motivations.................................................................... 71

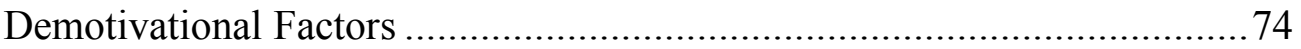

Satisfaction ....................................................................................8 80

Factors that Contribute to Satisfaction .................................................... 81

Factors that Detract from Satisfaction................................................. 84

Summary ................................................................................. 89

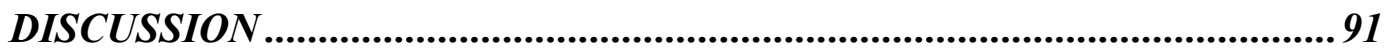

Interpretation of the Findings .......................................................93

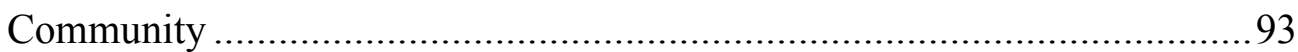

Assumptions .............................................................................. 96

Career Motivation and Preparation ....................................................... 101

Implications for Research and Practice ................................................104 


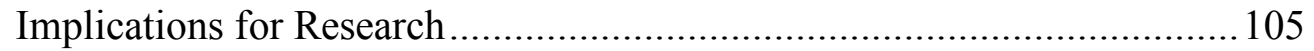

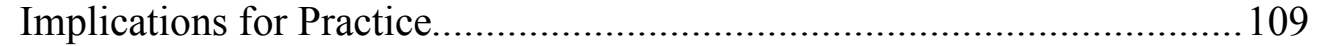

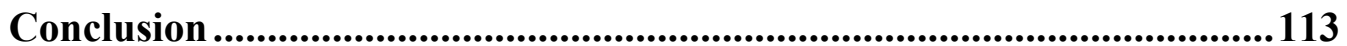

References........................................................................................... 116

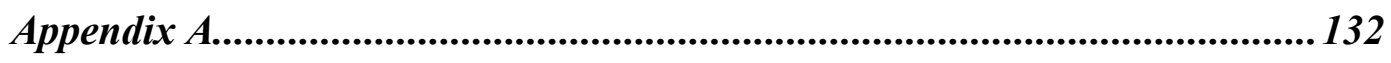

Appendix B............................................................................................ 133

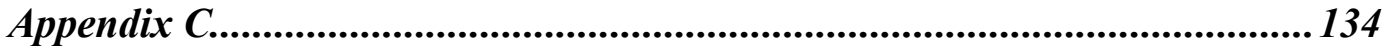

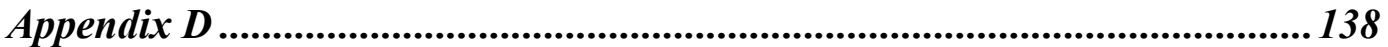

Appendix E............................................................................................. 139

Appendix F................................................................................... 140

Appendix G ...................................................................................... 141

Curriculum Vitae ..................................................................................................144 


\section{LIST OF TABLES}

Table Page

1. Survey Sample........................................ 38

2. Focus Group Sample........................................ 40

3. Quantitative Variables...................................... 41 


\section{LIST OF FIGURES}

FIGURE PAGE

1. Self-Determination Theory.............................. 32 


\section{INTRODUCTION}

Approximately $50 \%$ of students who begin doctoral programs will never complete them (Rigler et al., 2017; Sowell et al., 2015). These high attrition rates come at extreme costs to institutions. According to Smallwood (2004), reducing doctoral attrition by $10 \%$ would save an institution over $\$ 1$ million a year in doctoral stipends alone. Similarly, for the students themselves, the effects of leaving a doctoral program can negatively affect their self-esteem, lead to reduced employment opportunities, and discourage future academic study (Lovitts, 2001). The costs associated with attrition have been a longstanding issue within doctoral education (de Valero, 2001; Lipschutz, 1993), and the COVID-19 pandemic brought renewed urgency to reducing these costs. In a time of already reduced public funding for higher education, the pandemic caused colleges and universities nationwide to compete for enrollments and restructure their organization due to reduced state and federal funding and other unanticipated resource scarcities (Murakami, 2020). It is also possible that the future may bring additional threats to the doctoral education model or to doctoral student success. Shifts in funding models (Wichmann-Hansen \& Jesper, 2017), career preparation and skill development (Heflinger \& Doykos, 2016), and doctoral student populations (Hyle \& Goodchild 2014) suggest that knowledge of part-time students' experiences will be helpful in shaping successful doctoral models for the emergent environment. Although issues of attrition face all 
doctoral students, they may be particularly important for part-time PhD students, a type of nontraditional student.

\section{Nontraditional Doctoral Students}

Nontraditional students are notoriously difficult to define, and the nontraditional label is not without its issues. There is little consensus on its definition (Chung et al., 2014) and there have been calls to abolish the label completely to avoid alienating students or defining them by their differences (Gulley, 2016). Nontraditional is also a label typically applied to undergraduate student populations, which is reflected in many definitions using age as a defining characteristic. Horn and Carroll's (1996) definition, for instance, refers to nontraditional students as "older than typical" (p. 4), and Chung et al.'s (2014) review of the nontraditional term found that $78 \%$ of the definitions they reviewed used age as a defining category, with 25 being the most common cutoff point.

For doctoral students, age is a far less useful marker of nontraditional status, as older students may still pursue doctoral degrees in otherwise traditional ways (e.g., enrolling full-time, receiving assistantships). Other definitions that focus on student characteristics and how these characteristics affect their interaction with the university are far more applicable. Knowles (1984), for instance, argued that nontraditional students have defining characteristics that link them together, such as identities tied to their life experiences, an ability to take on extra responsibility, self-efficacy, and a resistance of requirements they perceive as inapplicable to their goals. Similarly, Lane (2004) defined non-traditional students as those who complete their degrees with one (or typically several) of the following factors: full-time employment, part-time study, dependent care, or financial independence. Unlike definitions that focus on relatively arbitrary markers 
such as age, Lane's definition emphasizes how external factors affect a student's participation within their academic programs.

Following Lane's definition, part-time $\mathrm{PhD}$ students can be considered nontraditional students. Unlike doctoral students enrolled in professional doctorates (e.g., Doctor of Education, Doctor of Business Administration), which frequently encourage part-time study (Offerman, 2011), part-time PhD students are often in research doctoral programs typically designed for full-time students (Archbald, 2011). That is, they experience their academic programs in nontraditional ways because their part-time enrollment (and typically additional factors) prevent them from engaging with their doctoral programs in the ways that the programs originally intended. This misalignment may cause part-time PhD students to experience difficulties that students in professional doctoral programs do not (Gardener \& Gopaul, 2012). While the research about part-time $\mathrm{PhD}$ student attrition remains sparse, existing studies indicate that part-time enrollment may impede progress to degree (Gittings et al., 2018; Ott \& Markewich, 1985). These studies are further bolstered by the extensive research about persistence in other nontraditional student populations, such as undergraduate (e.g., Forbus et al., 2011) and master's students (e.g., O’Toole et al., 2003; O’Connor \& Cordova, 2010),

Despite the documented challenges of part-time PhD study (Gardner \& Gopaul, 2012), the part-time PhD student population in the United States continues to grow (Hyle \& Goodchild, 2014). According to the US Department of Education (2016), 38.8\% of $\mathrm{PhD}$ students in the United States enroll on a part-time basis, and these rates are even higher for some disciplines. Part-time enrollment in PhD programs in Higher Education, for example, outpace the national average, with a recent study showing that part-time 
$\mathrm{PhD}$ students in that field of study outnumber full-time students three-to-one (Hyle \& Goodchild, 2014). The shifting landscape of higher education in light of COVID-19 may further contribute to this growth; historically, recessions have led to increased enrollments when individuals seek to improve their career prospects (Barr \& Turner, 2013). If these students are going to comprise $75 \%$ of some programs' populations (Hyle \& Goodchild, 2014), then greater attention needs to be paid to how part-time PhD student experiences differ from those of their full-time counterparts and more accommodations may need to be made in order to help part-time $\mathrm{PhD}$ students achieve success.

Motivation to pursue a doctoral degree and subsequent satisfaction with the degree program can be important factors in understanding the persistence of doctoral students (Bair \& Haworth, 1999; Gardner, 2008; Ivankova \& Stick, 2007; Lovitts, 2001). Mason (2012) found that motivation and satisfaction were significantly and positively correlated among doctoral students, noting that "graduate student program satisfaction is the critical factor for motivation to continue [to graduation]" (p. 271). Knowledge generated from studying motivation and satisfaction in part-time $\mathrm{PhD}$ students could contribute knowledge about why these students are pursuing $\mathrm{PhDs}$ on a part-time basis and which factors contribute to their decisions to persist.

Motivation, or the reason(s) for regulating behavior (Litalien et al. 2015), is a common construct in the literature about why students pursue doctoral degrees and why they decide to leave (Bair \& Haworth, 1999). Broadly, satisfaction can be understood as the fulfillment of an individuals' expectations and aspirations. This study focused on student satisfaction specifically, which Dericks et al. (2019) defined as an "overall positive attitudinal response to an educational experience" (p. 1050). Student satisfaction 
is of growing interest in the pursuit to understand student persistence (Dericks et al., 2019; Neumann \& Rodwell, 2009). Overall, the literature indicates persistence may be related to the relationship between motivation and satisfaction for doctoral students, yet no studies have examined how this relationship may differ for part-time $\mathrm{PhD}$ students.

\section{Study Purpose}

This study had two purposes: (a) investigate the relationship between motivation and satisfaction among part-time $\mathrm{PhD}$ students, and (b) build knowledge about the parttime PhD student experience.

\section{The Problem}

Historically, the literature about doctoral education has treated part-time $\mathrm{PhD}$ students as a problem (Dressel \& Mayhew, 1974; Gardner \& Gopaul, 2012). In 1974, for instance, Dressel and Mayhew categorized part-time doctoral students in the discipline of higher education as a "problem" for the field and a "weakness" of doctoral programs (p. 118). They posited that these students were only seeking employment credentials, and therefore programs with high proportions of part-time students would not maintain the intellectually rigorous standards necessary in doctoral education. While many professional doctoral degrees are focused on career preparation and structured in a way to accommodate, or even encourage, part-time study (Offerman, 2011), those involved in shaping research doctorate programs have historically rejected professional application of the degree outside of academe (e.g., through pursuing non-faculty careers), arguing that overt professionalization will detract from the pursuit of new knowledge and lead to the de-intellectualization of the $\mathrm{PhD}$ (Richardson \& Walsh, 1978; Brubacher \& Rudy, 2004). 
Beyond these speculative concerns, data indicate that there may be other issues with parttime study.

\section{Financial Issues}

More recently, programmatic shifts away from part-time study are likely to be motivated by financial reasons (e.g., programs admitting only fully funded students) or concerns about over producing PhDs in limited academic job markets that cannot accommodate them (Cassuto, 2013). According to the National Science Foundations' Survey of Earned Doctorates, these concerns are not unfounded. The Survey of Earned Doctorates (SED) is commonly regarded as the most comprehensive and accurate source of data for contemporary doctoral education (Okahana, 2019), and the 2019 report, which is the most recent available, documented that doctoral degrees have been awarded at a steadily increasing rate since the survey began in 1958. While initial surveys in 1958 showed fewer than 10,000 doctorates being awarded per year, in 2019 over 55,000 doctorate degrees were awarded, an increase of $450 \%$ over 60 years (National Science Foundation, 2019).

Perhaps more alarming than the growth of doctoral degrees are the financial and employment outcomes that many graduates receive. The most recent SED further found that $43 \%$ of doctoral recipients held graduate school debt at a mean rate of $\$ 26,137$. However, this debt is much higher for some fields, such as education $(M=\$ 47,672)$ and psychology/social sciences $(M=\$ 43,439)$. Many of these graduates may not have a salary commitment to combat their debt, as the survey results demonstrated that fewer than $50 \%$ of doctoral recipients secured academic employment commitments upon finishing their doctorates (National Science Foundation, 2019). Taken together, these data reveal a 
misalignment between the traditional understanding of doctoral education as preparation for faculty careers (Brubacher \& Rudy, 2004) and the current reality of many doctoral recipients graduating with substantial debt and not securing academic appointments (National Science Foundation, 2019). In this way, the SED calls attention to the problematic relationship between contemporary doctoral education and employment outcomes.

\section{Career Preparation}

The problematic nature of this relationship may be even stronger for part-time $\mathrm{PhD}$ students. Although the SED does not collect data on enrollment status, the scant research about part-time $\mathrm{PhD}$ students shows that these students are more likely to pursue alternative-academic (alt-ac) careers (e.g., administration, think-tank research, non-profit work) than they are to pursue faculty appointments (Hyle \& Goodchild, 2014). The false dichotomy of faculty versus alt-ac careers overlooks the variety of careers that share similarities with faculty work while technically qualifying as alt-ac. For example, someone who works as a full-time researcher in an academic staff or non-university affiliated role may spend their career conducting and publishing research in ways that align with faculty work but would not maintain a faculty appointment. That said, there are substantial differences between preparing for faculty and non-faculty careers (Kelly et al., 2020). For instance, training for faculty positions frequently requires hyperspecialization and primarily solitary work (depending on discipline), while alt-ac work typically requires generalists who work collaboratively on team-based projects (Kelly et al., 2020). This type of collaborative work requires many skills that faculty work may not (e.g., project management, conflict resolution), and recent research has shown that 
graduate education is largely insufficient in preparing doctoral students for alt-ac positions (Heflinger \& Doykos, 2016).

At the same time, studies suggest that career motivation is significantly predictive of satisfaction among doctoral students (Sakurai et al., 2017) and career competence and advancement are a common motive for all doctoral students (Templeton, 2016; Holmes et al., 2016). Taken together, the findings from these studies indicate that career motivation may be critical to doctoral student persistence, yet part-time students may not receive the career preparation such as mock interviews, job placement committees, and other forms of academic career preparation that full-time students pursuing faculty careers typically receive as compulsory parts of the $\mathrm{PhD}$ curricula of many departments (Turner, 2018; Heflinger \& Doykos, 2016). Moreover, the differences in vocational values between doctoral programs and part-time students may result in insufficient supports for these students (Baker \& Pifer, 2015), and doctoral students who feel unsupported are less likely to persist to graduation (Greene, 2015).

\section{Academic Integration}

Beyond issues of professionalization and employment, other scholars have documented the academic challenges and difficulties associated with part-time doctoral study. When compared to full-time doctoral students, part-time students are less likely to engage with the research community (Neumann \& Rodwell, 2009; Teeuwesen et al., 2014), to be socialized and integrated into the scholarly community (Deem \& Brehony, 2000; Gardner \& Gopaul, 2012), and to have faculty perceive them as scholarly or committed to their studies (Nora \& Snyder, 2007; Smith, 2000). In short, the literature about these students focuses primarily on the problematic aspects of part-time doctoral 
studies, both for the students who pursue these degrees and the programs and disciplines in which they enroll.

Yet while research about full-time doctoral students has grown exponentially in the past twenty years (Pifer \& Baker, 2016; Freeman et al., 2013), part-time PhD students are rarely the subjects of scholarly inquiry. Studies that center on part-time PhD students explicitly have focused more on the Australian and British contexts rather than the United States (e.g., Neumann \& Rodwell, 2009; Teeuwsen et al., 2014; Bates \& Goff, 2009). When controlling for peer-reviewed, published articles in the United States, only two studies were not restricted to a specific field of study: Gardner and Gopaul (2012) and Zahl (2015). These studies share several similarities, as they are both qualitative studies with ten part-time student participants from varied disciplines. To date, I have been unable to locate any quantitative studies that focus solely on part-time $\mathrm{PhD}$ students in the United States.

Given this lack of research, the "problem" (Dressel \& Mayhew, 1972, p. 118) with part-time PhD students may not come from their lack of dedication or poor academic performance, but rather from a lack of empirical evidence about why these students pursue doctoral degrees part-time and how this decision affects their overall satisfaction with their doctoral journey. What is known, however, is that part-time $\mathrm{PhD}$ students are likely experiencing significant challenges, as numerous studies have noted the difficulties of doctoral study and the effects that these difficulties can have on persistence (Pifer \& Baker, 2016; Bair \& Haworth, 1999; Nettles \& Millet, 2006). In addition, many of the challenges associated with poor persistence, such as stress and balancing external obligations, can be exacerbated for part-time undergraduate (Forbus et 
al., 2011) and master's students (O’Connor \& Cordova, 2010), suggesting the likelihood that they are experienced by part-time PhD students as well.

In order to advance knowledge of part-time PhD student persistence, I

investigated how students' motivation to enroll in and complete $\mathrm{PhD}$ programs on a parttime basis affects their overall satisfaction in their doctoral programs. More specifically, this study employed the Motivation for PhD Studies scale (MPhD; Litalien et al., 2015), the Doctoral Student Satisfaction scale (DSS; Dericks et al., 2019), and eight qualitative focus groups to understand the relationship between motivation and overall satisfaction of part-time PhD students through the lens of Self-Determination Theory (SDT; Deci \& Ryan, 1985).

\section{Research Questions}

Using an explanatory sequential mixed-methods approach, I answered three related research questions:

1. Does motivation for doctoral studies influence overall doctoral program satisfaction for part-time $\mathrm{PhD}$ students?

2. How do part-time $\mathrm{PhD}$ students describe their motivation for persisting and pursuing in their doctoral programs?

3. How do part-time $\mathrm{PhD}$ students describe their overall satisfaction with their doctoral programs?

\section{Study Significance}

These research questions have scholarly and practical significance. In terms of scholarly contribution, this study contributes to two bodies of literature: doctoral education and non-traditional student persistence. Current issues in doctoral education 
center around concerns of employability and the ever-decreasing availability of tenuretrack faculty positions (Wisker et al., 2019). This study contributes to this research stream through analyzing the effects of career-driven motivation on overall student satisfaction for part-time students, as specific items in the instrument asked about the influence of career motivation (see Appendix C), and career motivation was a frequent topic of discussion in the focus groups. Additionally, both the study of doctoral education and the literature about nontraditional student populations largely exclude part-time $\mathrm{PhD}$ students. While the former is heavily focused on full-time students, the latter is focused primarily on undergraduate students, and thus this study will contribute new knowledge to both research streams. Including part-time $\mathrm{PhD}$ students within empirical research about doctoral education will help the field of higher education better understand the needs of this specialized student sub-group and help build the foundation for further research into part-time $\mathrm{PhD}$ student experiences.

Practically, the research generated from these questions will begin building evidence to shape the work of faculty and administrators directly involved in the day-today practice of doctoral education. Doctoral education affects all facets of higher education, as doctoral students shape the future of research, education, administration, and policy (Bair \& Haworth, 1999). As such, it is critical to understand the factors that lead to doctoral student success and the issues that these students face. Through enabling all stakeholders in doctoral education to better understand part-time $\mathrm{PhD}$ students and the factors that lead to their success, this research will work to improve the daily practice and long-term success of doctoral education. Furthermore, this work investigates whether the motivation of part-time students for completing doctoral degrees aligns with the goals of 
the programs that are admitting them and whether these students are supported in ways that allow them to achieve success. In this way, this study works towards filling the request from administrators to find new and creative ways to bolster doctoral student success (Council of Graduate Schools, 2012).

\section{Summary}

This chapter provided an overview of the problems facing part-time $\mathrm{PhD}$ students and explained the rationale for conducting this study. I demonstrated that because parttime doctoral students are a growing student population (Department of Education, 2016), stakeholders involved in doctoral education (e.g., faculty, administrators, students) need further empirical research to help guide decision-making around doctoral admissions and program development, and strategies for supporting this student population. In the next chapter, I review the literature about part-time $\mathrm{PhD}$ students. 


\section{LITERATURE REVIEW}

My review of the literature is guided by the work of Jones (2013), who, in a thematic analysis of 995 papers written between 1971 and 2012 on issues in doctoral studies, found that there are six central themes to how doctoral education has been studied over the past 50 years: teaching, doctoral program design, writing and research, employment and career, student-supervisor relationship, and the doctoral student experience. Building off the work of Jones, I begin my literature review with reviewing these six areas and incorporating scholarship from the past eight years since their publication.

Given that this study is focused specifically on the last theme, the doctoral student experience, I review that topic in greater depth. Jones identified six sub-themes within the doctoral student experience, and I provide full reviews for each sub-theme: progress, student support, socialization, individual development, motivation, and discrimination and equity. Although Jones does not include satisfaction as a specific category within their review of the doctoral student experience, I include it in this review of the literature as the construct is relevant to my study, and it is related to the other themes identified by Jones.

After reviewing how doctoral education and the part-time $\mathrm{PhD}$ student experience have been studied, I close this chapter with a review of the literature about the theoretical framework that guided my study, Self-Determination Theory (SDT; Deci \& Ryan, 1985). 


\section{Doctoral Education}

This portion of the chapter reviews the literature about doctoral education according to the first five themes identified by Jones (2013): teaching, doctoral program design, writing and research, employment and career, student-supervisor relationship.

\section{Teaching}

Given that the etymology of doctor derives from the Latin word docere, meaning "to teach" (Latin Dictionary, n.d.), it is perhaps surprising that issues related to teaching account for only 3\% of the overall issues reviewed in Jones' (2013) work. This lack of research about teaching in doctoral education is typically traced to the emphasis on research and publishing in doctoral programs, which in turn is connected to how research and publishing are valued in academic careers and used as measures for promotion and tenure (Gaff \& Pruitt-Logan, 1998; Jepsen et al., 2012). However, the model of doctoral education that prioritizes research skills at the expense of teaching-focused curriculum may not be serving contemporary doctoral students. According to Wulff et al. (2004), the importance of teaching skills extends beyond the limits of the classroom, and thus decisions on how to prioritize teaching within doctoral education should avoid narrow considerations of the applicability and importance of teaching skills. Rather than a singular focus on classroom interactions, teaching should be conceptualized to include a wide variety of faculty responsibilities, including advising, curriculum development, and course and program assessment (Gibson, 1992; Wulff et al., 2004).

Without targeted development of these skills, many doctoral students who pursue faculty positions may be unprepared to successfully navigate their future careers.

Although the deleterious effects of this lack of preparation may be most obvious for those 
students who pursue careers in teaching-intensive positions with high course loads, Prewitt (2005) argues that the widespread devaluing of teaching in doctoral education also does a disservice to students who are able to secure tenure track positions at research intensive universities, noting that "although [they] are prepared to do original research, they seldom are adequately prepared for their teaching duties or their more general professional obligations" (p. 26). The consequences of removing teaching from the curriculum may be even stronger for part-time students who choose to pursue faculty careers, as part-time students are unlikely to obtain teaching assistantships (Gardner \& Gopaul, 2012). The exclusion of teaching skills from the curriculum may result in them finishing the doctorate with limited pedagogical knowledge, putting them at a distinct disadvantage in the faculty career search. They may also experience disadvantages due to the design of their doctoral programs.

\section{Doctoral Program Design}

Due to the substantial differences between disciplinary requirements, institutional funding, and programmatic culture, issues related to doctoral program design are inherently difficult to summarize. The expansive range of topics that fall into this category led Jones (2013) to delineate ten sub-topics within this larger theme, which include program and university policies (admissions, funding, assessment, scholarships), program and curricular structure (delivery, methodology, scope, topic selection), and the doctorate's wider applicability in contemporary work environments (professional

doctorates, linkage with practice and industry). While all of these areas affect part-time students, just as they do full-time students, an area of particular concern in the current 
global environment shaped by the COVID-19 pandemic is how program delivery shapes doctoral student experiences.

Doctoral programs have been operating in online delivery formats since the 1990s, yet there remains considerable debate over how comparable online programs are to their in-person counterparts. While some studies have shown that academic outcomes, such as cumulative GPA and exam scores (Mu et al., 2014), and reported support systems (Riedling, 1997) are equivalent across delivery formats, others call attention to the inherent ways that online programs differ from face-to-face program, such as diminished access to faculty (Thompson et al., 2018), overreliance on peer support (Berry, 2017), and technology-induced anxiety (Bollinger et al., 2012).

Likely due in part to the documented differences between delivery formats, research has shown that there is a stigma associated with online doctoral degrees. For instance, in one study $90 \%$ of faculty participants said they would not consider a candidate with a doctorate from an online institution for a faculty position in their program (Karl \& Peluchette, 2013). Radda and Mandernach (2012) argue that perceptions of online inferiority are rooted in a disconnect between traditional models of doctoral education as faculty preparation and evolving demands for doctoral degrees among practice-focused students (e.g., those adhering to a scholar-practitioner model). Regardless of reason, debates around program delivery will continue to impact part-time PhD students, as part-time students across degrees tend to pursue online options at higher rates than full-time students (Chen et al., 2010). 


\section{Writing and Research}

First coined in 1932, the maxim that one must publish or perish asserts that academic success is inextricably connected to an individual's ability to frequently publish in respected venues, such as top-tier academic journals (Coolidge, 1932; Rawat and Meena, 2014). While there are undeniable benefits to encouraging high productivity rates among faculty (e.g., institutional recognition, increased knowledge generation), Rawat and Meena (2014) draw attention to the many deleterious aspects of prioritizing publishing above all other aspects of faculty careers, noting that the pressure to publish has led to unethical practices among researchers (e.g., duplicate publishing), an overproliferation of academic journals, and a diminished focus on teaching and advising.

Yet despite the documented consequences of prioritizing publishing above all else, the pervasiveness of the pressures to publish have extended beyond faculty careers into doctoral education. Across disciplines, many doctoral students are now expected to publish scholarly articles prior to graduation, and some leading programs have made this publication a requirement of graduation (Lei \& Chuang, 2009). While it makes sense from a faculty perspective to acclimate doctoral students to the publishing process before they begin faculty careers, the pervasive pressure to publish as a doctoral student may cause additional obstacles for part-time students. The challenges of publishing frequently in well-respected journals have been well documented (Padmalochanan, 2019), and given the time constraints of completing a doctorate while working full-time, part-time students may be particularly disadvantaged at meeting the challenges associated with publishing. Additionally, because many part-time students may pursue alt-ac careers, the focus on 
publishing may detract from other areas of the doctoral experience that are more applicable to their needs, such as applying research to practice-based problems.

\section{Employment and Career}

When doctoral programs began in the United States in the late 1800 s and early 1900s, it was largely assumed that students would go on to pursue faculty careers, and thus curriculum focused on preparing them as teachers and researchers (Archbald, 2011). Yet since this time, the availability of academic jobs has declined and the number of PhDs produced has grown (Dickey, 2019; National Science Foundation, 2019). The discrepancy between doctoral degrees awarded and the availability of tenure track jobs have led doctoral education scholars to reassess how contemporary doctoral programs are - or are not_- preparing students for the careers they will move into after graduation. Gold and Dore (2001), for instance, conducted a national study of over 4,000 doctoral students in a range of programs and disciplines. Across these categories, they found a disconnect between what doctoral students wanted from their programs and what their programs were able to provide. They saw this issue manifest most prominently in terms of career preparation, noting that doctoral programs maintain a focus on preparing students for faculty positions at research universities, despite the continued diminishing availability of these positions and the reality that many students will leave academia for industry-based work. Although this study was conducted more than 20 years ago, the availability of academic jobs has continued to decline (Dickey, 2019), creating a stronger impetus for examining the relationship between doctoral education and industry application. 
The shift towards industry application in many doctoral programs is driven by more than the increasing scarcity of academic positions. Rather, a focus on industry relations may be a result of changes in knowledge production, increased collaboration and connection between universities, government, and industry, and larger changes in labor markets for doctorate recipients (Thune, 2010; Assbring et al., 2017). Some scholars have pushed against the professionalization of the $\mathrm{PhD}$, arguing instead for the expansion of professional doctorates in order to meet the needs of industry (Archbald, 2011). Arguments in favor of professional doctorates largely discount the prestige associated with the $\mathrm{PhD}$, as this prestige may not transfer to newer or less known doctoral degrees and may cause individuals seeking prestige to opt for the $\mathrm{PhD}$ regardless of other doctoral options (Deering, 1998; Townsend, 2002). Ultimately, more work needs to be done on the relationship between $\mathrm{PhD}$ education in the twenty-first century and career preparation. As doctoral student numbers continue to rise and available faculty positions continue to decline (Flaherty, 2020), the nature of this relationship becomes of paramount importance for all doctoral students, regardless of their enrollment status.

\section{Student-Supervisor Relationship}

The importance of the student-supervisor relationship to doctoral student success appears frequently in the literature. Early research in this area indicates that although doctoral students believe that one's relationship with their advisor is critical to their overall successful completion of the degree, they are also frequently disappointed in their own relationships with their doctoral advisors (Bargar \& Mayo-Chamberlain, 1983). More recently, research has shown the numerous positive outcomes that can come from a doctoral student being satisfied with their advisor relationship, including timely 
completion of the degree (Lovitts, 2011), successful disciplinary socialization (Gerholm, 1990; Weiss, 1981), and positive departmental culture (Hartnett, 1976). Conversely, unsatisfactory advisor relationships can be a contributing factor in a doctoral student's withdrawal from the program (Golde, 1996; Lovitts, 2011). In extreme instances, the breakdown of the student advisor relationship has resulted in either self-inflicted or faculty-directed violence (Burd, 1996; Hall, 1998). For all of these reasons, cultivating a positive advisor-advisee relationship should be a top priority for both students and faculty.

For part-time students, the advisor relationship may be particularly critical, as part-time students typically have reduced exposure to other faculty members and thus may turn to the advisor for mentoring in addition to advising roles. Yet many part-time students report having little or infrequent contact with their advisors. Without a strong relationship to rely on, these students typically turn to family members and friends to obtain support and advice about their doctoral education (Gardner \& Gopaul, 2012). In the vast majority of instances, it is unlikely that alternative supports are able to provide a level of guidance equivalent to that of a faculty advisor, and thus part-time students relying on external sources for advising and mentoring may not experience the positive effects of a satisfactory advisor relationship and may be more likely to leave their doctoral programs before graduating.

\section{The Doctoral Student Experience}

Having reviewed the first five themes from Jones (2013), I now turn to their final theme: the doctoral student experience. I have structured this section according to six sub-themes that Jones identifies as comprising the doctoral student experience: progress, 
student support, socialization, individual development, motivation, and discrimination and equity. Additionally, I review the literature about doctoral student satisfaction in order to explain its inclusion as a construct in my study.

\section{Progress}

According to Jones (2013), progress encompasses not only time to completion and persistence, but also stress, anxiety, and student-life balance. Of these subcategories, persistence is a particularly significant issue within doctoral studies as doctoral persistence rates remain low (Council of Graduate Schools, 2007). Despite the prevalence of research about doctoral student persistence, Gardner and Gopaul (2012) noted the ways in which stress affects the persistence of part-time doctoral students remains significantly understudied, and to date, no studies have compared the factors that influence doctoral persistence between part-time and full-time students.

Ott and Markewich (1985) provide a baseline understanding of part-time $\mathrm{PhD}$ student persistence. They found in their logit analysis of the retention of 1,454 doctoral students that initial registration status (full-time or part-time) was the single greatest indicator of graduate student persistence, with full-time students being significantly more likely to persist to graduation. They argued that the results from their study may be indicative of full-time students being more committed to their goals or being more fully socially integrated into their departments than part-time students. While their study is useful for its inclusion of enrollment status in its quantitative analysis of factors that affect doctoral student persistence, it analyzes a dataset from over 40 years ago (19771979), and thus the generalizability to doctoral education in the 21 st century may be limited. 
Building upon their findings, other studies have found that the stressors influencing persistence may be worse for other underrepresented student groups when compared to their traditional counterparts, such as first-generation students (Gardner \& Holley, 2011; Holley \& Gardner, 2012) and women students in programs primarily comprised of men (Holahan, 1979). The latter studies call attention to the influence of identity characteristics on the part-time PhD student experience (Gardner \& Gopaul, 2012), as the effects of these identity characteristics may also be affecting part-time student progress (Baker \& Pifer, 2015), and may be indicative of varying motivations for completing the degree. All of these studies show a continued need for research about how to best support part-time $\mathrm{PhD}$ students.

\section{Student Support}

The literature about doctoral education overwhelmingly speaks to the needs of various types of student supports, with a lack of support being shown to lead to doctoral attrition (Lovitts, 2001). Although types of supports vary widely and can include institutional, financial, and academic supports (Greene, 2015), Baker and Pifer (2011) called particular attention to the importance of relationship-building and its subsequent effects on scholarly identity development. Pushing beyond the typical student-advisor dyad that is used in studying doctoral student support, Baker and Pifer pulled from the work of Tinto (1993) and Weidman et al. (2001) to argue for the importance of a holistic understanding of doctoral student support that includes family, friends, and former colleagues. In this way, they reveal the interconnected nature of doctoral study. Rather than the cliched image of an isolated scholar working alone, they argued the successful doctoral journey requires the support of extensive personal and professional networks. 
If interpersonal connection is critical to doctoral student success, then empirical evidence is needed to understand how doctoral student networks may be disrupted for part-time PhD students. Because part-time students frequently pair study with full-time employment (Choy \& Cataldi, 2006; Watts, 2008), they may have less access to peer supports, such as study groups, networking, and emotional supports (Gardner, 2008; Gardner \& Gopaul, 2012; Offerman, 2011;). Similarly, time restrictions may also inhibit the ability of part-time students to receive support from their advisors and other departmental faculty, such as advising, networking, and becoming involved in research activities (Deem \& Brehony, 2000; Murakami-Ramalho et al., 2013). Receiving fewer supports and being less integrated into the department may lead to feelings of otherness, which can prevent doctoral students from feeling accepted and supported by their departments and their disciplines more broadly (Pifer \& Baker, 2014). Although the primarily qualitative work around student supports has not examined the influence of lack of support on overall satisfaction, these studies build a strong foundation for further study into the ways that faculty, departments, and institutions may support part-time students and provide them with the tools necessary for success, including socialization into their programs and fields of study.

\section{Socialization}

Socialization is one of the most studied topics in doctoral education (Mendoza \& Gardner, 2010). Weidman et al. (2001) provided a widely accepted framework for how socialization occurs at the graduate level. Taking a holistic approach to understanding graduate student socialization, they suggest that this process is defined by knowledge acquisition, investment, and involvement. Through the process of socialization, students 
are supposed to learn the conventions of their disciplines, which will then prepare them for future academic careers. In this way, socialization is considered the first step in preparing future faculty members (Sweitzer, 2009) and is one of the most critical factors in understanding success in doctoral education (Weidman et al., 2001).

Yet for part-time students, time constraints caused by competing priorities such as full-time employment and dependent care may restrict their ability to engage in the types of experiences that lead to socialization (Gardner \& Gopaul, 2012). Dressel and Mayhew (1974) argued that "it is doubtful that part-time involvement in programs of higher education can accomplish such a scholarly or professional socialization" (p. 119), as the students have far fewer interactions with faculty and peers. Although Dressel and Mayhew seemed to be making assumptions about part-time $\mathrm{PhD}$ student involvement, Neumann and Rodwell (2009) provide empirical support for this argument through their analysis of large-scale survey data on Australian doctoral students. They found that parttime students are less satisfied with both the infrastructure and the intellectual climate of their doctoral programs than full-time students, and they posit these reduced levels of satisfaction may be a direct result of their lack of integration into these areas. These findings echo those of Deem and Brehony (2000), who argued that full-time students inherently have easier access to academic and peer cultures and thus are more fully integrated into the department than those who attend on a part-time basis.

The lack of socialization also affects the identity development of part-time students, as socialization is one of the ways students develop scholarly identities (Baker \& Lattuca, 2010). The diminished ability to develop a scholarly identity may come from a simple lack of exposure to research cultures (Deem \& Brehony, 2000). Teeuwsen et al. 
(2014) documented the issues that stem from a lack of access to research cultures

firsthand, as two of the three authors are part-time doctoral students themselves. They reiterated the frequently documented problems associated with external obligations, noting these obligations inhibit their ability to engage in research beyond what is required of their courses. Having these research opportunities early in an academic program may be critical to developing the skills necessary to complete a research-driven degree and develop a researcher identity (Murakami-Ramalho et al., 2008), and therefore a lack of access can potentially lead to diminished satisfaction and persistence.

\section{Individual Development}

In addition to impeding the development of a researcher identity, part-time study may affect other identity-related issues. Gardner (2008) suggested that students who feel as if their identities are misaligned with what is expected of a typical doctoral student may be less likely to persist with their doctoral studies, leading to many underrepresented students leaving their programs before completing. Expanding beyond demographic markers of identity (e.g., race/ethnicity, gender), Baker and Pifer (2015) suggested that part-time students may feel torn between their professional identities and their academic identities, particularly if they are pursuing an alternative-academic (alt-ac) career path, as their professional goals and values may misalign with those of the academy.

Although the research overwhelmingly shows that students who complete degrees part-time are likely working full-time, Offerman (2011) added to this picture through profiling what he called "the nontraditional doctoral degree student" (p. 21). In his review of the literature, he finds that nontraditional doctoral students are typically employed in mid-career level positions and have years of experience in their professional 
roles. Yet when they return to the classroom, their professional identity can be subsumed by their academic identity, which he argued is likely to be less developed than their professional identity. Therefore, the tension between their academic and professional identities may cause conflicts in how they see themselves within the classroom (Offerman, 2011). Similarly, Watts (2008) has argued that balancing the effects of competing commitments can lead to a "fractured student identity" (p. 369), as part-time students are forced to constantly switch between their various identities (e.g., student, employee, parent).

The work of both Watts (2008) and Offerman (2011) raise questions about how these identity issues influence the motivation and satisfaction of part-time students, as they draw attention to identity development as a potential further obstacle these students face in persevering through their doctoral programs. However, both authors provide only theoretical discussions of the issue, and empirical research is needed to fully understand the identity development of these students and how this development affects their doctoral experiences.

\section{Motivation}

The additional effort and stress required for pursuing a $\mathrm{PhD}$ part-time (Gardner \& Gopaul, 2012) calls attention to the motivations of students pursuing these degrees. Studies have demonstrated that traditional doctoral students are motivated by external factors such as their relationship with their advisors, prospects of faculty careers, academic achievement and goal setting; on the other hand, they are motivated by internal factors such as self-development, personal interest in the subject matter, and internal dedication (Brailsford, 2010; Reamer 1990; Lovitts, 2001). Motivation has further been 
connected to various academic outcomes. High motivation has been shown to lead to positive outcomes such as academic achievement, persistence, and scholarly productivity, while low motivation has been linked to leaving programs prior to graduation and poor academic achievement (Kahn \& Schlosser, 2010; Morrison \& Lent, 2014; Pintrich, 2003; Bair \& Haworth, 1999).

In doctoral studies, motivation is typically studied qualitatively and studies tend to focus on the connection between motivation and persistence (Bair \& Haworth, 1999). When it is studied quantitatively, researchers tend to treat motivation as a unidimensional construct and thus represent it with a single item in their instruments (e.g., Lovitts, 2001; Pauley et al., 1999). Motivation has become so prevalent in the literature about doctoral education that Litalien et al. (2015) argued that understanding the motivation of students may be the critical factor in helping doctoral students succeed and achieve their personal goals. Yet, as Litalien et al. (2015) documented, studies of motivation are not typically grounded in theoretical frameworks, an issue that they saw as stemming from its overtreatment as a unidimensional, rather than multidimensional, construct.

The relationship between motivation and positive or negative outcomes may also be connected to the ways doctoral students view themselves and their work. Gardner (2008) found that doctoral students who view themselves in an overall positive manner are more likely to complete their dissertations than those who view themselves negatively. Supporting Gardner's findings, other studies have shown how negative student characteristics, specifically negative emotions, thoughts, and behaviors, can negatively impact doctoral student motivation. For instance, Muszynski (1988) conducted a qualitative study of 120 doctoral students and found that factors that negatively impact 
mental health (depression, stressful life-events, isolation) impeded student motivation and consequently affected overall progress to degree completion. Other negative behaviors that have been shown to negatively impact doctoral student motivation include procrastination (Solomon \& Rothblum, 1984; Golde \& Dore, 2001; Gardner, 2009), and fear of failure (Rothblum et al., 1986). Although these studies did not focus on the effects of motivation on satisfaction specifically, many of these negative outcomes are likely to result in low levels of overall satisfaction (Barnes \& Randall, 2011).

While these studies provide a useful overview of doctoral student motivation and satisfaction, they are focused primarily on traditional, full-time, doctoral student populations. Their applicability to part-time students may be limited, as part-time students face significant challenges that full-time students do not and therefore may be pursuing graduate degrees for reasons beyond those typically ascribed to full-time students (Gardner \& Gopaul, 2012). To date, there have been no studies that have examined the motivations of part-time doctoral students. That said, part-time students are likely to be particularly motivated by advancing their careers and serving as role models for their families, as they are typically older, mid-career professionals, who have family responsibilities (Offerman, 2011; Choy \& Cataldi, 2006).

However, the career focused aspect of part-time students' potential motivation raises questions about whether their programs are preparing them for the careers they intend to pursue, which may be more likely to be alt-ac rather than traditional faculty appointments (Hyle \& Goodchild, 2014). Heflinger and Doykos (2016), for instance, found in their logit analysis of doctoral students at one private research university that students felt well prepared in professional areas associated with traditional academic 
work (e.g., writing, research, presentations), but poorly prepared in areas more associated with alt-ac careers (e.g., leading teams, negotiating with supervisors). Although their study did not account for effects of enrollment status, it raises questions about whether part-time $\mathrm{PhD}$ students are being prepared for the careers that might have motivated their enrollment and whether this perceived lack of preparation influences their overall satisfaction.

\section{Satisfaction}

Satisfaction is a frequently studied construct in doctoral education and numerous studies have shown correlations between high levels of satisfaction and doctoral student persistence (Bair \& Haworth, 1999). Nyquist and Woodford (2000) identified seven personal and institutional areas that may lead to dissatisfaction among doctoral students:

(a) lack of preparation for faculty careers, (b) anticipated quality of faculty careers, (c) narrow definitions of professional work, (d) lack of faculty mentors, (e) mistrust of mentor advice, (f) reduced or unstable funding, and (g) inability to understand their work in a larger global context. Although dated, their work shows a longstanding concern about future employment among doctoral students and thus supports the possibility of the connection between motivation and overall satisfaction.

Other research about satisfaction has focused on the relationship between satisfaction and positive or negative outcomes in a student's academic career. In both undergraduate and graduate postsecondary education, for instance, satisfaction has been connected to a number of positive outcomes, including increased motivation (Donohue \& Wong, 1997; Deci \& Ryan, 2000), retention (Roberts \& Styron, 2010), completion rates (Neumann \& Rodwell, 2009), academic performance (Pike, 1993), and overall well-being 
(Diener et al., 1999). At the doctoral level, studies tend to focus on factors that contribute to student satisfaction, such as advisors (Zhao et al., 2007; Ives \& Rowley, 2005), departments (Morton \& Thornley, 2001; Umbach \& Porter, 2002; Golde, 2005), and peers (Deem \& Brehony, 2000; Pilbeam et al., 2013).

These studies tend to study satisfaction as it relates to a specific factor (e.g., satisfaction with one's advisor), rather than on satisfaction with the doctoral student experience as a whole. Yet isolating satisfaction within a specific element of the doctoral journey may not provide a full understanding of the ways these varying elements (advisor, department, peers) contribute to overall satisfaction and its associated positive outcomes, such as well-being and success (Dericks et al., 2019). Further, studying satisfaction with specific elements only may obscure the connection between satisfaction and perceptions of discrimination and equity.

\section{Discrimination and Equity}

Jones (2013) defined the category of discrimination and equity as studies about doctoral issues that are concerned with the rights of underrepresented populations and establishing equity. Issues of discrimination and equity have been documented in doctoral education since it began in the United States, when it was intended only for White, upper class men (Goodchild, 1996). Studies on discrimination and equity in doctoral education focus primarily on inequities based on race/ethnicity (Crumb et al., 2019; Felder et al., 2014; Solorzano, 1998; Nettles, 1990), and gender (Holahan, 1974; Holmstrom \& Holmstrom, 1974; Espino et al., 2010; Barthelemy et al., 2016; Maher et al., 2004). Other studies document the effects these inequities can have on scholarship (Bell, 2009), 
socialization (Felder et al., 2014; Turner \& Thompson, 1993), and persistence (Crumb et al., 2019; Maher et al., 2004; King \& Chepyator-Thompson, 1996).

Issues of equity are particularly pertinent to the part-time $\mathrm{PhD}$ student experience, as part-time students report largely feeling minoritized and inferior when compared to full-time students (Gardner \& Gopaul, 2012). Similarly, Muraki-Ramalho et al. (2013) found in their qualitative study of full-time and part-time educational administration doctoral students that many part-time students report feeling that faculty members are penalizing them for having external commitments to their doctoral studies, and that these perceived penalties lead to them feeling isolated from the research community.

From the faculty perspective, perceptions of inequity may stem from the relationships students build with their faculty members. Faculty may perceive part-time students as less engaged academically and therefore inferior to their full-time students (Curran, 1987; Nora \& Snyder, 2007). Gardner (2008) suggested that a perceived lack of engagement may stem from part-time students not exhibiting the trait of "intensive devotion to a subject" (p. 36) that faculty expect to see in doctoral-level study. Given that many faculty members were full-time students themselves, this perceived lack of devotion to the topic may be indicative of faculty expecting to see students mirror their own experiences (Hyle \& Goodchild, 2014). This perception can lead to part-time students feeling isolated and alienated (Gardner \& Gopaul, 2012).

A perceived lack of belonging is further exacerbated by the logistics of student funding. The majority of funds available to attend conferences and travel for research are set aside exclusively for full-time students (Nora \& Snyder, 2007). Many part-time students must fund this travel through either personal or alternative methods, or, more 
likely, not attend. Similarly, part-time students may feel alone in their navigation of the doctoral student experience, as "there is no map" on how to complete a $\mathrm{PhD}$ part-time, despite the extensive resources and recommendations that exist for full-time students (Gardner \& Gopaul, 2012, p. 72). The discrepancies between resources available to fulland part-time students can lead part-time students to feelings of otherness (Gardner \& Gopaul, 2012), dissatisfaction with the doctoral student experience (Nettles \& Millet, 2006), and guilt when they are unable to devote their full attention and efforts to other various aspects of their lives (Gardner \& Gopaul, 2012).

Taken as a whole, the literature about doctoral education and part-time student experiences reveals that part-time $\mathrm{PhD}$ students likely experience significant challenges in completing their doctoral degrees, yet very little research has identified and explored those challenges. The challenges of part-time doctoral study raise questions about their motivations for pursuing doctoral degrees and their overall satisfaction with completing degree programs that are typically designed for the needs of full-time students. In order to work towards understanding both the motivations and satisfaction of part-time $\mathrm{PhD}$ students, this study was grounded in the theoretical framework of Self-Determination Theory (SDT; Deci \& Ryan, 1985).

\section{Theoretical Framework}

Self-Determination Theory (SDT) posits that humans are motivated by three innate needs that must be satisfied for overall well-being: competence, autonomy, and relatedness with others (Deci \& Ryan, 1985). While autonomous motivation (performing a task under one's own volition) may satisfy the three needs, controlled motivation (performing a task due to external pressure) may impede them. Ideally, autonomous 
motivation leads to improved performance, engagement, and overall well-being. However, achieving autonomous motivation is impacted by both proximal interpersonal contexts (e.g., relationships with others) and distal contexts (e.g., sociocultural conditions). Thus, SDT analyzes motivational states of individuals within their larger social contexts in order to make predictions about emotional, physical, and psychological health (Deci \& Ryan, 2012).

Pushing against historical tendencies to treat motivation as a unitary concept (e.g., Bandura, 1996; Hull, 1943), Deci and Ryan $(1985,2012)$ viewed motivation as a continuum, with autonomous self-determined motivation on one end and controlled nonself-determined motivation on the other. Along this continuum, there are five types of regulatory behaviors that align with motivation, three of which are autonomous (intrinsic, integrated, identified) and two of which are controlled (introjected, external) (Deci \& Ryan, 2012):

1. Intrinsic: interest and enjoyment from performing an activity for its own sake;

2. Integrated regulation: performing an activity because it aligns with personal values, goals, and needs;

3. Identified regulation: performing an activity because one believes it is important;

4. Introjected regulation: performing an activity due to internal pressure for recognition or to avoid shame; and

5. External regulation: performing an activity for a reward or to avoid punishment. 
This framework guided my study in several important ways. Most prominently, SDT provided an operational definition of motivation that I used throughout the quantitative and qualitative portions of the study. Quantitatively, the survey employs the Motivation for PhD Studies Scale (MPhD), and this scale is grounded within SDT (Litalien et al., 2015). Qualitatively, the types of motivation and their associated regulatory practices guided protocol development (see Appendix D) and subsequent data analysis. For instance, when coding my qualitative data, I used SDT to craft my initial codebook (Collins \& Stockton, 2018).

Additionally, the relationship between motivation and satisfaction served as the impetus for my chosen variables and study design. According to SDT, extrinsic and intrinsic motivation are on a continuum, and as an individual moves from extrinsic to intrinsic motivation more of their innate needs of competence, relatedness, and autonomy are satisfied (see Figure 1). Meeting all of these needs then leads to increased overall well-being (Deci \& Ryan, 2012). Feelings of well-being have also been associated with overall satisfaction (Diener et al., 1999) and persistence (Deci \& Ryan, 2012). This relationship is further supported in the literature applying SDT, which largely finds that extrinsic motivation is connected to lower levels of satisfaction than intrinsic motivation (Vansteenkiste et al., 2007; Zhang et al., 2011). 


\section{Figure 1}

\section{Self-Determination Theory (Ryan \& Deci, 2000)}

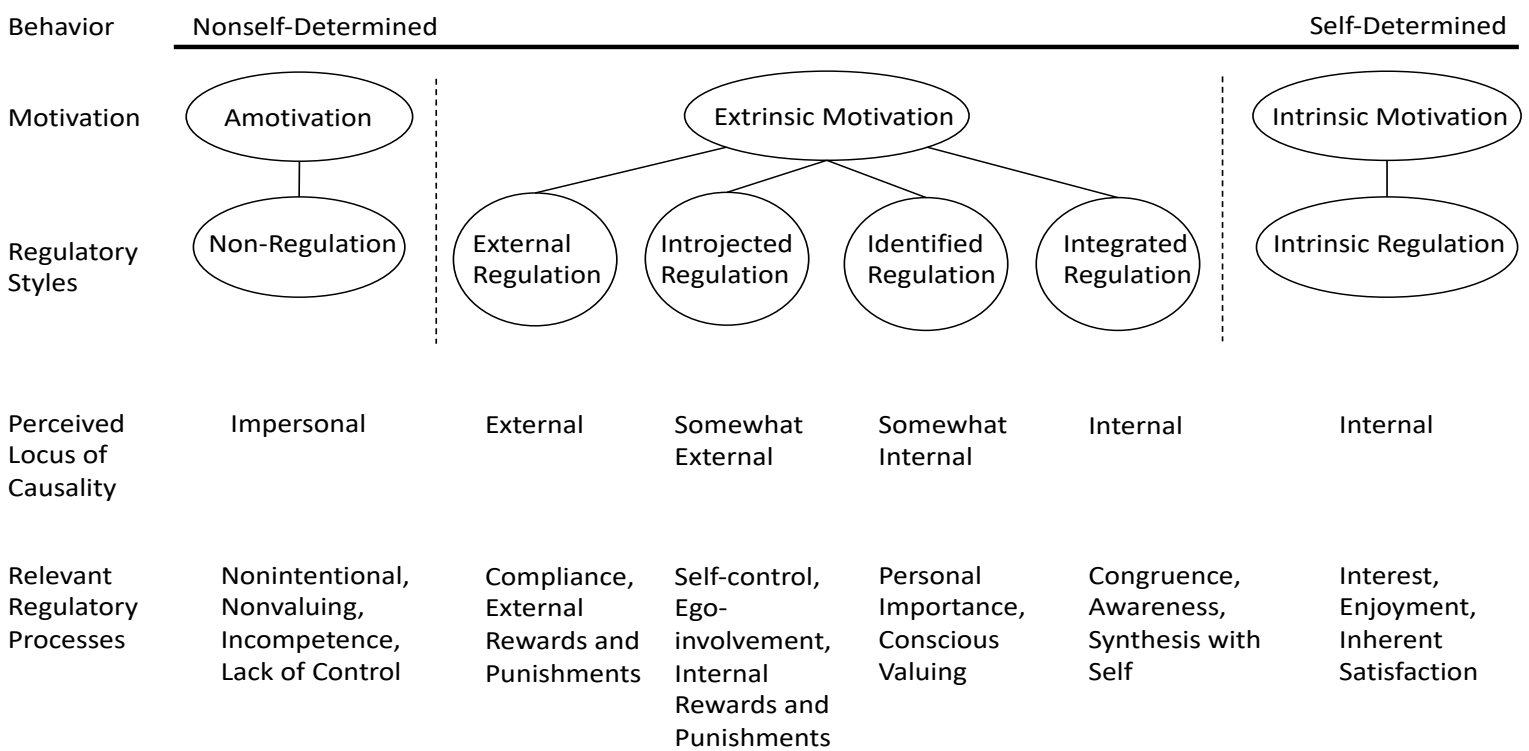

In this study, I tested the applicability of SDT to studying the part-time $\mathrm{PhD}$ experience through examining the influence of motivations on overall satisfaction. Based on the theory, I expected to see lower levels of satisfaction for those who report high levels of controlled motivation and higher levels of satisfaction for those who report high levels of autonomous motivation. Given the lack of research on this topic, my study design reflects the need to understand the potential nuances that exist between motivation and satisfaction for this student population through supplementing the quantitative relationship between the variables with qualitative focus groups.

\section{Summary}

In this chapter, I reviewed the literature related to part-time PhD students through the framework of Jones (2013). Through doing so, I showed there are significant gaps in the literature about part-time $\mathrm{PhD}$ student experiences. The majority of existing research adopts a deficit mindset when studying this student population, as many of the existing 
studies note the problems and issues associated with part-time study without discussion of benefits or opportunities. I closed the chapter with a review of my theoretical framework and an explanation of how this framework guided my data collection and analysis. In the following chapter, I expand upon this explanation through reviewing the methodology and research design of the study. 


\section{METHODOLOGY AND RESEARCH DESIGN}

This study used an explanatory sequential mixed methods approach to examine the influence of motivation on overall student satisfaction among part-time $\mathrm{PhD}$ students (see Appendix B for alignment of study purpose, research questions, theoretical framework, instrumentation, and data analysis). In explanatory sequential mixed method designs, research begins with quantitative data collection, and quantitative data is then used to guide data collection in the subsequent qualitative phase (Creswell \& PlanoClark, 2011). The first research question (Does motivation for doctoral studies influence overall doctoral program satisfaction for part-time $\mathrm{PhD}$ students?) was answered quantitatively using multiple linear regression. As discussed in further detail below, I

then used the results of the regression to finalize the design of the qualitative phase of my study. In this qualitative phase, I answered the second and third research questions (How do part-time $\mathrm{PhD}$ students describe their motivation for persisting and pursuing in their doctoral programs?; How do part-time PhD students describe their overall satisfaction with their doctoral programs?).

My rationale for choosing a mixed methods design was rooted in the recommendations of Greene et al. (1989). They argued that the decision to mix methods should be based on two factors: (a) the relationship between the qualitative and quantitative data, and (b) how the problem had been studied in the past (see Appendix A for full alignment of my study with the recommendations of Greene et al., 1989). 
Research on the part-time PhD student experience is limited, and previous studies have typically taken a qualitative approach (e.g., Gardner \& Gopaul, 2012; Zahl, 2015). While these qualitative works have built a foundational understanding of the part-time $\mathrm{PhD}$ student experience, a mixed methods approach allows for a more complete understanding of the research problem (Creswell, 2012). In my study, I chose to use mixed methods in order to provide a more comprehensive understanding of the relationship between motivation and satisfaction than either a quantitative or qualitative study would allow. The quantitative portion enabled me to statistically test whether selfdetermination theory held true for a large percentage of the part-time students at one institution, while the qualitative portion added nuance to the quantitative findings through hearing directly from the students themselves.

\section{Study Design}

My decision to utilize an explanatory sequential mixed methods approach was further based on the qualitative and quantitative research strands. Research strands, as Creswell and Plano Clark (2011) explained, encompass the full processes of quantitative and qualitative data collection and analysis, from posing questions to interpreting results. Therefore, mixed method designs are based on four key decisions surrounding the use of quantitative and qualitative strands: (a) the level of interaction between the strands, (b) the relative priority of the strands, (c) the timing of the strands, and (d) the procedures for mixing the strands (Creswell \& Plano-Clark, 2011; see Appendix E for alignment between research strand decisions and study design).

\section{Relationship between Quantitative and Qualitative Data}

In sequential mixed methods studies, the quantitative portion of the study informs the subsequent qualitative data collection and analysis. In my study, the relationship 
between the quantitative and qualitative phases differed from what I had originally intended. During the preliminary planning phases of this study, I intended to collect data from part-time students only. However, when preparing for data collection in the quantitative phase of the study, I noticed discrepancies in the list of student contacts I received from the Office of Institutional Research, with some students who I knew to be part-time being listed as full-time and vice versa. These discrepancies made me realize that, like many aspects of doctoral education enrollment status can change from semesterto-semester, and thus I needed study participants to self-identify which enrollment status (full-time or part-time) best captured their doctoral experience. Therefore, I identified a need to send the survey to all $\mathrm{PhD}$ students.

At this point in my study, my intention was to divide students based on their enrollment status after receiving survey results and only use part-time students in both the quantitative and qualitative portions. In making this decision, my assumption was that the quantitative phase would shape the qualitative phase through altering the focus group protocol based on the findings from the survey data. For example, if I had found that parttime students reported higher levels of controlled motivation than autonomous motivation, I would have revised the qualitative protocol to focus more heavily on understanding controlled motivations.

However, after I finalized the survey data, I ran a regression model with the full sample out of curiosity, and I was surprised by the findings. Contrary to what I expected to see based on the literature about part-time doctoral student experiences, I found that enrollment was not a significant predictor of satisfaction (see Chapter 4 for full findings). Based on this finding, I saw a need for further investigation into the relationship between motivation, satisfaction, and enrollment status. Therefore, rather than exclude full-time 
students from my quantitative analysis, I decided to use the full sample for the quantitative phase and also to include full-time students in the qualitative phase.

In this way, I used the quantitative phase to shape the qualitative phase through restructuring my study to include comparative elements. Specifically, in the quantitative phase, I ran the regression using both full-time and part-time students and controlled for enrollment. In the qualitative phase, I coded and analyzed the full-time student focus group responses to identify ways that part-time student responses differed (see below for full data analysis processes). Although comparison was not a specific intention in my initial design, the purpose of explanatory sequential mixed methods is to be flexible in shaping the qualitative phase based on the quantitative phase, and thus this decision is aligned with explanatory sequential mixed methods (Creswell and Plano-Clark, 2011). In addition to restructuring the study based on the quantitative findings, I made other changes to the qualitative phase based on the results of the quantitative phase. While I did not alter my protocol based on the quantitative findings in the way that I had initially planned (i.e., changing questions based on specific factors affecting motivation and satisfaction), I did make slight changes to its structure. Specifically, I re-organized the questions from three categories (motivation, enrollment, satisfaction) into two (motivation, satisfaction). Due to the change in qualitative participants, I also reworded one of the questions to accommodate part-time and full-time students. Finally, I decided to provide participants with an online form for them to provide additional comments anonymously, in case they had something relevant that they did not feel comfortable sharing in a group setting with both full- and part-time students (see Appendix D for full protocol). 


\section{Sample}

The population for this study was all PhD students currently enrolled at one public research university in the mid-western portion of the United States, hereafter known as the University. This sample included 1,200 PhD students, of which $553(46 \%)$ were enrolled part-time during the Spring 2020 semester. Although data received from the Office of Institutional Research indicated the enrollment status of participants during the Spring 2020 semester, all participants were asked to self-identify their enrollment status. The decision to have students self-identify their enrollment was due to the possibility that their Spring 2020 enrollment status was not reflective of their overall doctoral program enrollment. Given the length of doctoral study, it is possible that some full-time students may enroll on a part-time basis at some point during their doctoral career, and conversely some part-time students may be briefly enrolled full-time.

Following procedures of convenience sampling (Dillman et al., 2014), eligible students from all disciplines were included, but those pursuing non-research doctorates (e.g., EdD) were excluded, as the research questions focus specifically on understanding the experiences of research doctoral $(\mathrm{PhD})$ students. That said, there are debates in the field about the structural and philosophical differences between $\mathrm{EdD}$ and $\mathrm{PhD}$ programs in education, and thus there may be more similarities than differences between these degree programs (Martinez-Lebron, 2016). However, examining the differences between $\mathrm{EdD}$ and $\mathrm{PhDs}$ is beyond the scope of this study, and thus only $\mathrm{PhD}$ students are included here. Although the $\mathrm{PhD}$ student experience will differ considerably between fields of study (Golde, 2005), I did not exclude students based on discipline, as my study's purpose was to examine the overall relationship between motivation and satisfaction for part-time $\mathrm{PhD}$ students and build knowledge about the part-time $\mathrm{PhD}$ student experience. 
The University does not currently offer online $\mathrm{PhD}$ programs, and thus all students were enrolled in face-to-face programs, although nearly all students were completing work fully-online during the time of the study due to the COVID-19 pandemic. The survey was open from September 9, 2020 through October 9, 2020; focus groups began 11 days after the survey closed and were conducted over a period of 10 days (see below for full discussion of data collection procedures).

For the qualitative portion of the study, the sample came from survey participants who indicated on the survey they would be willing to participate in follow-up focus groups. After the survey closed, I emailed all students who indicated they would either be willing to participate in focus groups or that they wanted to learn more about this phase in the study $(N=257)$. These students were asked to indicate their general availability during the period of qualitative data collection (October 20, 2020 - October 30, 2020). Students were assigned to focus groups based on their indicated availability and their enrollment status.

\section{Survey Sample}

Descriptive statistics were conducted to examine characteristics of the data. Data was disaggregated according to key demographics identified in the literature about doctoral education as being influential to the doctoral student experience: gender, race/ethnicity, enrollment status, doctoral stage, and doctoral programs according to Biglan's (1973) classification schema (see Table 1). In this schema, Biglan (1973) categorizes academic disciplines into four groups according to their subject-matter and characteristics: (a) hard pure, exact and natural sciences; (b) hard applied, science-based professions; (c) soft pure, humanities and social sciences; and (d) soft applied, social science-based professions. The schema is based on overarching disciplinary differences. 
At the broadest level, hard disciplines follow a single common paradigm, while soft disciplines will employ a variety of methodologies and concepts. More specifically, hardpure disciplines focus on universals and simplifications, using an atomistic approach; soft-pure disciplines use a holistic approach to examine individual cases. Similarly, hardapplied discipline focus on applying knowledge to solve problems and create products and techniques with the ultimate goal of physical mastery, while soft-applied disciplines seek to improve professional practice through focusing on individual growth, reflection, and policies and procedures based on continual learning.

As shown, the majority of participants identified as men (man=55.4\%, woman $=43.3 \%$, other responses $=1.4 \%$ ) and as white $($ white $=63.3 \%$, minority $=36.7 \%)$. The majority of participants were from disciplines that Biglan (1973) classified as soft applied (53.5\%), and the majority were in the dissertation phase of their doctoral programs $(53.0 \%)$. This sample is skewed slightly towards the soft applied and soft pure doctoral programs, as $44 \%$ of the University's $\mathrm{PhD}$ programs are classified as soft applied, while 5\% are classified as soft pure. The racial and gender makeup of the sample closely mirror the demographics of the student population at the University. Enrollment is defined as those who identified as attending either "mostly" or "completely" part-time or full-time, and the sample distribution also closely mirrors that of the University.

\section{Table 1}

\section{Survey Sample Overview}

\begin{tabular}{lllc}
\hline Response Category & Response Options & $N$ & Percent of Responses \\
\hline \multirow{2}{*}{ Gender } & Man & 238 & 55.4 \\
& Woman & 186 & 43.3 \\
& Other Responses & 6 & 1.4 \\
\hline \multirow{2}{*}{ Race/Ethnicity } & African & 2 & 0.5 \\
& Asian & 50 & 11.6 \\
\hline
\end{tabular}




\begin{tabular}{lllr}
\hline & Black/African & 61 & 14.2 \\
& Hawaiian/Pacific & 1 & 0.2 \\
& Hispanic/Latino & 19 & 4.4 \\
Middle Eastern & 11 & 2.6 \\
& Native American & 1 & 0.2 \\
& Two or more & 9 & 2.1 \\
& White/Caucasian & 272 & 63.3 \\
& Other Responses & 4 & 0.9 \\
\hline Enrollment & Full-Time & 330 & 76.7 \\
& Part-Time & 100 & 23.3 \\
\hline Biglan's & Hard Applied & 89 & 20.8 \\
Classification & Hard Pure & 85 & 19.8 \\
& Soft Applied & 230 & 53.5 \\
\hline \multirow{2}{*}{ Doctoral Stage } & Soft Pure & 24 & 5.6 \\
& Coursework & 164 & 38.1 \\
& Comprehensive & 37 & 8.6 \\
& Dissertation & 228 & 53.0 \\
\hline
\end{tabular}

\section{Focus Group Sample}

Descriptive statistics were also conducted on focus group participants in order to provide an overview of participant characteristics. Variables in this analysis were the same as the quantitative phase (gender, race/ethnicity, enrollment status, doctoral programs according to Biglan's [1973] classification schema, and doctoral stage; see Table 2). As shown, participants primarily identified as women (woman $=62.0 \%$, $\operatorname{man}=36.0 \%$, other responses $=2.0 \%$ ) and as White (white $=72.0 \%$, minority $=26.0 \%$ ). The majority of participants were from disciplines that Biglan (1973) classified as soft applied $(70.0 \%)$, and in the dissertation phase of their doctoral programs (64.0\%). This sample was skewed towards soft applied disciplines, women, and white participants, as is discussed in the study's limitations.

\section{Table 2}

Focus Group Sample Overview 


\begin{tabular}{lllc} 
Category & Response Options & N & Percent of Responses \\
\hline Gender & Man & 18 & 36.0 \\
& Woman & 31 & 62.0 \\
& Other Responses & 1 & 2.0 \\
\hline Race/Ethnicity & Asian & 1 & 2.0 \\
& Black/African American & 8 & 16.0 \\
& Brazilian & 1 & 2.0 \\
& Hispanic/Latino & 1 & 2.0 \\
& Middle Eastern & 1 & 2.0 \\
& Native American & 1 & 2.0 \\
& White/Caucasian & 36 & 72.0 \\
\hline Enrollment & Full-Time & 30 & 60.0 \\
& Part-Time & 20 & 40.0 \\
\hline Biglan's Classification & Hard Applied & 3 & 6.0 \\
& Hard Pure & 8 & 16.0 \\
& Soft Applied & 35 & 70.0 \\
& Soft Pure & 3 & 6.0 \\
\hline Doctoral Program Stage & Coursework & 17 & 34.0 \\
& Dissertation & 32 & 64.0
\end{tabular}

\section{Instrumentation}

This study was based on two constructs common in the literature about doctoral education: motivation and satisfaction. I operationalized these constructs into three continuous variables (satisfaction, autonomous motivation, controlled motivation) using previously developed measurement scales, namely: the Doctoral Student Satisfaction Scale (DSS; Dericks et al., 2019) and the Motivation for PhD Studies Scale (MPhD; Litalien et al., 2015). The qualitative portion used a semi-structured protocol to guide the focus groups, and this protocol was refined based on the findings in the quantitative portion (Creswell \& Plano-Clark, 2011; see Appendix C for the quantitative instruments, Appendix D for the qualitative protocol, and below for a full discussion of data collection procedures).

\section{Quantitative Instrument}


Doctoral student motivation and satisfaction were measured based on the selection of pre-existing scales, namely: the Motivation for PhD Studies scale (MPhD; Litalien et al., 2015); the doctoral student satisfaction scale (DSS; Dericks et al., 2019). Enrollment status was dummy coded and used as an independent variable. Other independent variables (autonomous motivation, controlled motivation) came from the two factor-structure of the $\mathrm{MPhD}$ scale, and the dependent variable (satisfaction) was from the DSS (see Table 3). Other demographic and doctoral program characteristics were also collected and used for descriptive purposes in order to provide an overview of the study's sample (see Appendix C for full instrument). 
Table 3

\section{Quantitative Variables}

\begin{tabular}{|c|c|c|c|}
\hline Variable & Recorded & Level of Measurement & Study Use \\
\hline Enrollment Status & $\begin{array}{l}\text { Dummy Coded } \\
(0=\text { full-time } \\
1=\text { part-time })\end{array}$ & Categorical & $\begin{array}{l}\text { Independent } \\
\text { Variable }\end{array}$ \\
\hline $\begin{array}{l}\text { Autonomous Motivation } \\
\text { (intrinsic, identified, } \\
\text { integrated) }\end{array}$ & Total Score & Continuous & $\begin{array}{l}\text { Independent } \\
\text { Variable }\end{array}$ \\
\hline $\begin{array}{l}\text { Controlled Motivation } \\
\text { (introjected, external) }\end{array}$ & Total Score & Continuous & $\begin{array}{l}\text { Independent } \\
\text { Variable }\end{array}$ \\
\hline Overall Satisfaction & Total Score & Continuous & $\begin{array}{l}\text { Dependent } \\
\text { Variable }\end{array}$ \\
\hline
\end{tabular}

Doctoral Student Satisfaction Scale. The Doctoral Student Satisfaction Scale (DSS; Dericks et al., 2019) is a 10-item lexical measure designed to measure doctoral student satisfaction. In this instrument, Dericks et al. (2019) offered an understanding of overall doctoral student satisfaction that holds satisfaction as a unitary affective construct. That is, they viewed student satisfaction not as occurring within specific elements of the doctoral experience (e.g., the advisor relationship) but rather as an "overall feeling towards an overall education experience" (Dericks et al., 2019, p. 1050). Given the lack of empirical evidence about part-time student motivation, I chose this unitary scale for this study in order to establish a baseline understanding of overall satisfaction and lay the foundation for future work into the specific areas that may influence part-time PhD student satisfaction.

The developers tested the validity of this scale through an exploratory principal component analysis. They found the scale to be unidimensional as there was no factor loading below .73, and a single component accounted for $67 \%$ of the variance. Similarly, they tested for reliability of the scale, and Cronbach's alpha of internal consistency 
reliability was .94 and therefore above the recommended threshold of .8 (Dericks et al., 2019). DSS measures satisfaction on a six-point Likert scale. Participants are asked to rate whether they agree or disagree that the items align with their overall $\mathrm{PhD}$ experience, with response options ranging from "Strongly Disagree" to "Strongly Agree." Of the ten items total, five items are scored positively (Good, Enjoyable, Satisfactory, Excellent, Happy) and five items are scored negatively (Unhappy, Bad, Terrible, Disappointing, Unsatisfactory) (Dericks et al., 2019).

Motivation for PhD Studies Scale. The Motivation for PhD Studies Scale (MPhD; Litalien et al., 2015) is a 15-item measure of doctoral students' motivation as defined by self-determination theory (SDT; Deci \& Ryan, 1985). Unlike previous scales of doctoral motivation that treat motivation as a single dimension (e.g., Ivankova \& Stick, 2007; Lovitts, 2001), Litalien et al. (2015) created the MPhD through grounding motivation within the theoretical framework of SDT and thus conceptualized it as a multidimensional construct existing of varying types and degrees of motivation. As such, $\mathrm{MPhD}$ contains five subscales based on types of regulatory behavior from SDT (intrinsic, integrated, identified, introjected, external), and each subscale contains three items. These items fall into a two-factor higher order structure of autonomous motivation (intrinsic, integrated, identified) and controlled motivation (introjected, external). The five subscales represent a continuum between high to low self-determination, beginning with intrinsic and ending with external regulation (Litalien et al., 2015).

Because these scales are on a continuum, they are expected to be more highly correlated with the scales that fall most closely to them. For example, intrinsic should be highly correlated with integrated and potentially negatively correlated with external. The autonomous scales (intrinsic, integrated, identified) are typically associated with positive 
outcomes (e.g., persistence, well-being) while the controlled scales (introjected, external) are typically associated with negative outcomes (e.g., anxiety, rote learning). Following the recommendations of Litalien et al. (2015), I used these scales to create two independent variables according to the two-factor higher order structure: autonomous motivation (intrinsic, integrated, identified) and controlled motivation (introjected, external). All scales were measured on a five-point Likert scale. Participants were asked whether statements correspond to their experiences, with response options ranging from "Does Not Correspond at All" to "Corresponds Exactly" (Litalien et al., 2015).

Litalien et al. (2015) analyzed the reliability and validity of the MPhD scale with two different samples $(N=244, N=1060)$, as well as a combined analysis that included all participants $(N=1304)$. The developers examined reliability among the subscales of both samples and found the scores from the scale were reliable. Reliability estimates were computed using McDonald's (1970) omega, as this allowed the researchers to look at the strength of the association between constructs as well as item-specific measurement errors. The first/second sample estimates for scale score reliability were: .79/.73 (intrinsic), .85/73 (integrated), .69/60 (identified), .73/78 (introjected) and .85/.81 (external). With the exception of identified, these scores are all above the recommended .70 range for scale score reliability (Litalien et al., 2015). Although identified is slightly below this range, the score is on a three-item scale and thus should be interpreted cautiously, as the number of items on a scale can notably influence McDonald's omega (Sijtsma, 2009; Streiner, 2003).

Additionally, Litalien et al., (2015) tested the measurement invariance of the fivefactor structure (intrinsic, integrated, identified, introjected, external) and the two-factor higher-order structure (autonomous, controlled) among diverse groups of $\mathrm{PhD}$ students 
who varied according to age, gender, citizenship, academic program, and program progression. The developers found that the measurement model was fully invariant across samples, and that measurement invariance was supported across both samples as well as across the subgroups of the demographic variables (e.g., age, gender; Litalien et al., 2015).

Demographics and Doctoral Program Characteristics. In addition to the $\mathrm{MPhD}$ and the DSS, my instrument included several questions related to demographics and doctoral program characteristics. These items allowed me to gain a detailed overview of the quantitative and qualitative samples. Demographic questions included common identity questions (gender, race/ethnicity, age, marital/familial status) as well as other questions focused on doctoral programs (doctoral program stage, academic program, employment status, doctoral funding, employment goals). The total instrument included 5 demographic items, 8 doctoral program characteristic items, 15 items on the MPhD scale, and 10 items on the DSS, for a total of 38 items (see Appendix C). All data were anonymized and stored on a secure server to protect participant identities.

\section{Qualitative Instrument}

The qualitative instrument was a focus group interview protocol that consisted of four questions. Two questions asked about doctoral student motivation, and two questions asked about doctoral student satisfaction. As discussed, the protocol was finalized after quantitative data was analyzed with hierarchical multiple regression (Creswell \& Plano-Clark, 2011). Specifically, the regression results indicated that enrollment was not a significant predictor of satisfaction, and this finding led me to include full-time students in the qualitative phase of the study. Due to this change, I removed a question from the protocol that asked about the students' decision to enroll on 
a part-time basis. Instead, all questions were asked so they were applicable to both fulland part-time student participants (see Appendix D for full protocol).

\section{Data Collection}

Following recommendations from Creswell and Plano-Clark (2011) for explanatory sequential mixed methods designs, data collection occurred in two phases, beginning with the quantitative phase and ending with the qualitative phase.

\section{Quantitative Data Collection}

Methods for administering the survey were based on those of the Washington State University Doctoral Student Experience Survey (WSU DSES; Dillman et al., 2014). I chose to model the instrument distribution off of this survey due to the similarities between the surveys (studying doctoral students at one institution), and the high response rate the WSU DSES achieved. Although typical student survey response rates range between $20 \%$ and $30 \%$, the WSU DSES received a response rate of $77 \%$ (Dillman et al., 2014). Although the DSES collected data both digitally and in hard-copy, I revised this format into a digital only distribution due to the effects of COVID-19 on mail distribution and student work environments (e.g., most students working remotely). Surveys were distributed digitally through the students' university email addresses over the course of 14 days and the survey was open for 30 days:

Day 1: An email was sent to all students asking them to complete the survey online and providing a link to complete the survey online

Day 7: A second email request was sent to nonresponders

Day 14: A final email reminder was sent to nonresponders

Day 30: The survey closed 


\section{Qualitative Data Collection}

The survey asked respondents if they would be willing to participate in a focus group related to their doctoral student experience. All students who indicated they would be willing to participate in this phase of the study were asked to complete an online scheduling form to indicate their availability. I then used the responses from this form to schedule and conduct eight semi-structured focus groups. I chose focus groups for the qualitative portion of this study as they are appropriate for studying attitudes and experiences and can generate data beyond what can be captured in individual interviews (Ravitch \& Carl, 2016). I further chose focus groups for the group dynamic they provide, as Ravitch and Carl (2016) suggest this group setting may enable participants to feel as if their experiences are valid and thus may encourage them to share more details than they would in one-on-one setting. Moreover, focus groups can provide a way to identify whether experiences are commonly shared or are extreme individualized cases (Patton, 2015).

Following the recommendations of Kreuger (2014), I designed focus groups to have between 5 and 8 participants. However, due to last minute scheduling limitations and the desire to include all willing participants in focus groups, two of the eight focus groups had nine participants. On account of the constraints of the COVID-19 pandemic, I conducted all focus groups virtually via Microsoft Teams. I used the recording feature available in Microsoft Teams to record the audio and video of all of the sessions. I scheduled each focus group for 60 minutes; however, four of the eight focus groups extended past this time and lasted between 65 and 90 minutes. 


\section{Data Analysis}

In the quantitative portion, I used hierarchical multiple regression to address the first research question. Subsequently, I used several coding techniques to analyze the qualitative data and address the second and third research questions (see Appendix B for full alignment between research questions and data analysis).

\section{Quantitative Data Analysis}

I began quantitative data analysis through using descriptive statistics to understand characteristics of the sample and the variables of interest (autonomous motivation, controlled motivation, satisfaction). As discussed, descriptive statistics were used to examine the sample according to key factors identified in the literature about parttime $\mathrm{PhD}$ student experiences. I further used descriptive and inferential statistics from the initial model to test whether the data violated assumptions of regression. I examined correlation coefficients to test for issues of multicollinearity, and all coefficients were below the .7 threshold. To test the assumption of linearity, I created a simple scatterplot of studentized residuals and unstandardized predicted values, which showed a linear relationship between the data. After running the initial regression model, I examined the Durbin-Watson statistic to test for independence of observations, and this statistic was within the accepted range of $\sim 2.0$. Subsequently, I inspected standardized residuals in order to identify potential outliers outside of $+/-3.0$ that may be affecting results (Cohen, 2008; Osborne, 2016).

After ensuring collected data did not violate any of the assumptions of regression, I analyzed quantitative data using hierarchical multiple regression, as this method is appropriate for understanding variance when the study contains a continuous dependent variable (overall satisfaction score) and categorical (enrollment) and continuous 
independent variables (autonomous motivation score, controlled motivation score) (Osborne, 2016). I chose regression for this analysis as it shows "the extent to which we can understand one variable based on another variable" (Osborne, 2016, p. 53). In other words, regression allowed me to examine whether doctoral student satisfaction is explained through autonomous and controlled motivation and whether this relationship is affected by enrollment status. Although Pearson's Product Moment Correlation would also provide an analysis of the relationship between these variables, regression has the added benefit of adding the intercept (the expected value of satisfaction when motivation is zero), the standardized and unstandardized slope (the effects of the independent variables on the dependent variable), and the error term (the difference between a student's predicted and actual satisfaction score). In this way, regression provides a more comprehensive understanding of the relationship between the variables and thus is preferable to Pearson's Product Moment Correlation (Osborne, 2016).

In my analysis, I entered variables into the model using two blocks. Block 1 contained enrollment status, and Block 2 added in autonomous motivation and controlled motivation. Using a block entry method allowed me to analyze whether the two motivation variables (autonomous, controlled) explained a significant amount of variance in overall satisfaction above and beyond that which was explained by enrollment status (Osborne, 2016). I evaluated the quality of the regression model using several key statistics. I used the $F$-statistic to determine whether the models were statistically significant, and I used $R$-square to determine how much variance was explained in each model. Finally, unstandardized regression coefficients were examined to identify the relationship between the dependent and independent variables (Cohen, 2008). 


\section{Qualitative Data Analysis}

Focus groups were all recorded using the built-in recording feature of Microsoft Teams, which captured both audio and video data. Following the completion of each focus group, Microsoft Teams emailed me the video file, which I saved in a secure cloudbased storage system (CardBox). Once all focus groups were complete, I submitted each individual file to an online transcription service, which processed the files and produced transcripts. After downloading the transcript for each focus group, I went through the transcripts and compared them to the audio files to check for discrepancies or errors. I found only minor errors and corrected them in the transcripts. I then went through the files and identified all of the speakers with pseudonyms in order to compare responses based on participant characteristics (e.g., enrollment status, discipline).

Once transcripts were complete, I uploaded all of the individual files into Quirkos, a qualitative coding and analysis program. After all files were added to the system, I began coding in three rounds: exploratory, first cycle, second cycle (Saldaña, 2016). In the exploratory round, I used holistic coding, which is a method used to "grasp basic themes or issues in the data by absorbing them as a whole [the coder as 'lumper'] rather than by analyzing them line by line [the coder as 'splitter']" (Dey, 1993, p. 104). This round involved coding large sections of my transcripts in order to gain a general understanding of the data and its alignment with both my quantitative findings and SDT more broadly. In this cycle, I coded data according to the types of motivation ("autonomous," "controlled") and the types of regulatory behaviors ("intrinsic," "integrated," "identified," "introjected," "external”) within SDT. I also coded according to factors that contribute to satisfaction ("satisfaction +") and those that detracted from it 
("satisfaction -"). This method was chosen as the first step for its ability to provide an overview of the data before first and second cycle coding began (Saldaña, 2016).

After exploratory holistic coding was complete, I began first-cycle coding using eclectic coding. Rather than being a coding approach itself, eclectic coding uses two or more other coding approaches simultaneously. I used three types of coding in this cycle: in vivo (using a word/phrase from the transcript; "I just want to get it done"), descriptive (summarizing the main point; "career advancement"), and values (inferring values, attitudes, or beliefs from the transcript; "faculty prefer full-time students") coding (Saldaña, 2016). Taken together, these three types of codes allowed me to examine what was said, how it was said, and how it might connect to larger values and beliefs held by participants. After first cycle eclectic coding was complete, I began second-cycle coding. Second-cycle coding involved developing themes from the exploratory and first cycle coding. This included grouping first-cycle codes and analyzing these groups in order to derive themes from the data ("knowledge acquisition," "avoiding perceived disappointment"). Subsequently, these themes served as the basis for the findings of the study (Guba, 1990; see Appendix F for full coding structure).

\section{Limitations}

There were four limitations of this study. This study was limited through its focus on $\mathrm{PhD}$ students at one institution and at one point in time. The experience of $\mathrm{PhD}$ students likely differs based on institution classifications, sectors, sizes, and regions. However, focusing on one institution allowed me to minimize the effects of these variables between institutions, as all students in this study had commonalities in their experiences within the one institution. Similarly, interviewing doctoral students at one point in time during their doctoral programs may have led to their reported motivation 
and satisfaction not being representative of the entirety of their doctoral experience postgraduation. That is, their levels of motivation and satisfaction may be disproportionately heightened or lowered due to recent incidents in their programs. However, interviewing people during their experiences may also provide more concrete examples of aspects of their doctoral experience that affected motivation and satisfaction that may otherwise be misremembered in a retrospective study. This limitation is particularly relevant to this study, as data were collected several months into the COVID-19 pandemic (September and October, 2020), and thus the effects of the pandemic may have skewed study participants' perceptions of their motivations and satisfaction. In other ways, the pandemic may have heightened existing issues within part-time PhD student experiences (e.g., lack of social supports). Future research should explore the motivation and satisfaction of part-time $\mathrm{PhD}$ students on a national scale at varying points in their doctoral journeys and, preferably, not during a pandemic.

Another limitation of this study was the choice of convenience sampling. Although convenience sampling is widely used in the social sciences, it is subject to issues with sampling errors and overall generalizability to target populations (Dillman et al., 2014). For instance, because students decide for themselves whether they will participate in the survey, certain subgroups of students may uniformly decide not to participate, thus leading to survey bias. However, increasing the response rate for the survey can help minimize these issues (Jager et al., 2017), and the survey received a higher than average response rate of $36.7 \%$ (Dillman et al., 2014). That said, the choice of convenience sampling may have led to the study sample being skewed towards the social sciences, which limited my ability to draw comparison between disciplines. Golde (2005) has argued that disciplinary differences are the single greatest factor in 
determining doctoral student experiences, and therefore future studies should seek greater participation from part-time PhD students in disciplines outside of the social sciences.

The focus group format may have also limited the responses received from some part-time $\mathrm{PhD}$ students, as focus group participants had substantial amounts of information to share within a limited time frame. Although several of the focus groups ran over time in order to allow everyone to share their experiences, it is possible that some students did not fully divulge their experience due to time constraints. Given that the majority of part-time participants had a great deal of information to share, future studies should consider individual interviews in order to allow for full discussion of parttime PhD student experiences.

Finally, this study is limited through my decision to not analyze demographic and identity characteristics. Although research about doctoral education shows that identity is a critical factor in understanding doctoral student experiences, my decision to exclude these factors from my analyses was based on the minimal amount of research that exists about the part-time $\mathrm{PhD}$ student experience. Although my qualitative data was skewed towards white participants and those who identified as women, I sought a sample that varied in personal identities (race, gender) and doctoral program characteristics (program, stage) in order to build a foundational understanding of the relationship between motivation and satisfaction in part-time $\mathrm{PhD}$ students. Subsequent research should examine how this relationship varies based on identity and demographic factors. 


\section{FINDINGS}

This chapter presents findings about motivation and satisfaction in part-time $\mathrm{PhD}$ students based on results from a survey of $430 \mathrm{PhD}$ students and eight focus groups with 50 participants. Of this sample, 100 survey participants and 20 focus group participants were enrolled on a part-time basis. Full-time student responses are included due to the explanatory sequential mixed methods study design, as the quantitative phase of the study showed that comparative data would aid in understanding the relationship between motivation and satisfaction for part-time students. Therefore, data from full-time participants were analyzed quantitatively and qualitatively for comparison purposes (i.e., to identify trends in the data that were common among part-time students only). However, because the study's purpose was to examine the relationship between motivation and satisfaction in part-time $\mathrm{PhD}$ students and build knowledge about the part-time $\mathrm{PhD}$ student experience, findings focus primarily on part-time student responses. The collection and analyses of the data were guided by Self Determination Theory (SDT; Deci \& Ryan, 1985), and thus SDT also guides the organization of this chapter (see Figure 1).

The chapter proceeds in two parts: quantitative results and qualitative findings. In the quantitative section, I analyzed survey data with hierarchical multiple regression in order to examine the relationship between satisfaction and autonomous and controlled motivation based on enrollment status. Quantitative results indicate that autonomous 
motivation leads to increased satisfaction and controlled motivation leads to decreased satisfaction, when controlling for enrollment. Enrollment is not a significant predictor of satisfaction; however, part-time students report higher levels of satisfaction than full-time students. Building off the quantitative results, I then present findings from analysis of the focus group data. Qualitative findings are structured according to SDT's two-factor (autonomous/controlled) structure (see Figure 1). Qualitative findings indicate that parttime $\mathrm{PhD}$ students are motivated by knowledge advancement and relationships with others, yet they may be lacking in access to community physically, psychologically, and intellectually. As with many full-time doctoral students, part-time students are demotivated when they feel isolated, exhausted, and overwhelmed; part-time enrollment frequently exacerbates these feelings. Motivation to enroll part-time is largely dictated by family circumstances (e.g., financial need) and a desire for career advancement. Like motivation, satisfaction is primarily derived through relationships with others, feeling supported, and personal and professional growth. Reduced access to academic and social communities may be a source of dissatisfaction, as findings indicate this lack of community frequently prohibits part-time student socialization and may also lead to untested assumptions regarding faculty roles and the purpose of research doctoral education. However, part-time students appear reticent to report feelings of overall dissatisfaction, despite indicating feeling dissatisfied with many components of the doctoral experience.

\section{Quantitative Findings}

Quantitative data were used to answer the first research question: Does motivation for doctoral studies influence overall doctoral program satisfaction for part-time $\mathrm{PhD}$ students? Quantitative data analysis began with running descriptive statistics on study 
variables and sample characteristics. The survey was sent to $1200 \mathrm{PhD}$ students and received a total of 441 responses, which is a response rate of $36.7 \%$. However, preliminary review of the data indicated that several of these responses were incomplete as data were not inputted for any variable beyond demographics. Therefore, as the first step in data analysis, I removed incomplete responses from the raw data $(N=11)$, which resulted in the final sample for analysis $(N=430)$.

\section{Multiple Regression}

I used hierarchical multiple regression to analyze the relationship between satisfaction and autonomous and controlled motivation according to enrollment status. Prior to running the regression model, I ran descriptive statistics on study variables to provide an overview of sample responses (see Table 2). As shown, the full-time students $(N=330)$ reported higher levels of autonomous motivation $(M=3.72, S D=.69)$ than controlled motivation $(M=2.82, S D=.83)$ and were more satisfied than dissatisfied $(M=4.45, S D=.96)$. Similarly, part-time students reported higher levels of autonomous motivation $(M=3.57, S D=.78)$ than controlled motivation $(M=2.54, S D=.89)$ and were more satisfied than dissatisfied $(M=4.68, S D=.79)$. As shown, full-time students reported higher scores on every variable except satisfaction (Full-time, $M=4.45, S D=.96$; Parttime, $M=4.68, S D=.79)$ 
Table 2

\section{Descriptive Statistics of Study Variables}

\begin{tabular}{llccccc}
\hline Enrollment & Variable & $N$ & Minimum & Maximum & Mean & $S D$ \\
\hline Full-time & Autonomous & 330 & 1.44 & 5.00 & 3.72 & .69 \\
& Controlled & 330 & 1.00 & 5.00 & 2.82 & .83 \\
& Satisfaction & 330 & 1.00 & 6.00 & 4.45 & .96 \\
\multirow{5}{*}{ Part-time } & Autonomous & 100 & 1.44 & 5.00 & 3.57 & .78 \\
& Controlled & 100 & 1.00 & 5.00 & 2.54 & .89 \\
& Satisfaction & 100 & 2.80 & 6.00 & 4.68 & .79 \\
\hline
\end{tabular}

Following these descriptive statistics, I ran hierarchical multiple regression in two blocks with the full survey sample $(N=430)$. Block 1 included enrollment status only, which was dummy coded as "0" for full-time and "1" for part-time. Subsequently, following Osborne (2016), I ran diagnostics on this model to ensure data did not violate the assumptions of hierarchical multiple regression. I ran correlations to test for multicollinearity and results indicated that no variables violated this assumption, as all were below the recommended threshold of 0.7 (Cohen, 2008). I plotted residuals and predicted values in a simple scatter plot to test for the assumption of homoscedascity, and I ran a histogram to test the assumption of normality. The data did not exhibit homoscedascity as the values were spread randomly with no clumping patterns. The data further revealed a normal distribution in a standard bell-shaped curve (Osborne, 2016). Diagnostics identified 7 cases (1.6\% of total sample) that had standard residuals outside of $+/-3$ standard deviations and thus could be considered outliers. According to Osborne (2015), cases with residuals outside of this range have only a $.13 \%$ chance of being "a legitimate member of the population of interest" (p. 104) and therefore these 7 cases were removed due to their influence on the model. 
I then reran the regression analysis with outliers removed $(N=423)$. Block 1 , which contained only enrollment status (full-time $=0$, part-time $=1$ ) resulted in a nonsignificant explanation of variance in satisfaction $F(1,421)=2.98, p=.09, R^{2}=.005$. As indicated, Block 1 showed that enrollment was not a significant predictor of satisfaction. In other words, the level of reported satisfaction of these participants was not significantly explained by their enrollment status (full-time, part-time). Subsequently, Block 2 included the two motivation variables, controlled and autonomous. Block 2 also included enrollment, as this inclusion controls for enrollment's effects on other model variables (autonomous motivation, controlled motivation). Therefore, the block entry method allowed me to examine the relationship between autonomous and controlled motivation and satisfaction will controlling for enrollment status.

Block 2 resulted in a significant increase in $R^{2}\left(R_{\text {change }}^{2}=.16\right)$, $F_{\text {change }}(1,419)=38.96, p<.05, R^{2}=.16$, indicating that autonomous and controlled motivation significantly explained variance in overall satisfaction. The unstandardized regression coefficients were .35 (autonomous) and -.22 (controlled), indicating that for every one-unit change in autonomous motivation, there will be a .35 unit increase in satisfaction, while for every one-unit change in controlled motivation, there will be a .22 unit decrease in satisfaction (see Table 3). To put it another way, data indicate that participants who are motivated by autonomous factors (e.g., personal enjoyment) are likely to have increased satisfaction, while participants who are motivated by controlled factors (e.g., avoiding shame) are likely to have decreased satisfaction. 
Table 3

Multiple Regression Coefficients

\begin{tabular}{|c|c|c|c|c|}
\hline Model & Variable & $\begin{array}{c}\text { Unstandardized } \\
\text { Regression } \\
\text { Coefficient }\end{array}$ & Standard Error & $p$-Value \\
\hline \multirow[t]{2}{*}{1} & Constant & .012 & .051 & .816 \\
\hline & Enrollment & .180 & .104 & .085 \\
\hline \multirow[t]{4}{*}{2} & Constant & .011 & .047 & .808 \\
\hline & Enrollment & .181 & .097 & .062 \\
\hline & $\begin{array}{l}\text { Autonomous } \\
\text { Motivation }\end{array}$ & .345 & .042 & .000 \\
\hline & Controlled Motivation & -.219 & .042 & .000 \\
\hline
\end{tabular}

I analyzed survey data using descriptive and inferential statistics to examine the relationship between doctoral student satisfaction and autonomous and controlled motivation across enrollment classifications. Descriptive statistics indicate that while fulltime students report higher levels of autonomous and controlled motivation, part-time students report higher levels of satisfaction. Results of analysis using hierarchical multiple regression show that enrollment status was not a significant predictor of satisfaction. Regression results further indicate that, when controlling for enrollment, autonomous motivation is a significant positive predictor of satisfaction while controlled motivation is a significant negative predictor of satisfaction. In the following section, I present findings from the qualitative phase of my study.

\section{Qualitative Findings}

The qualitative phase of my study allowed me to answer the second and third research questions: (a) how do part-time $\mathrm{PhD}$ students describe their motivation for persisting and pursuing in their doctoral programs? and (b) how do part-time $\mathrm{PhD}$ students describe their overall satisfaction with their doctoral programs? As discussed, 
qualitative data from full-time participants were analyzed in order to identify trends in variation between part-time and full-time experiences. The study's purpose is to examine the relationship between motivation and satisfaction in part-time $\mathrm{PhD}$ students and build knowledge about the part-time $\mathrm{PhD}$ student experience, and thus the findings presented here are based on part-time student responses. I begin with reviewing sources of autonomous motivation before discussing controlled motivations, demotivational factors, and overall reported satisfaction.

\section{Motivations}

According to Self-Determination Theory, motivations fall on a continuum between autonomous and controlled (Deci \& Ryan, 1985). Autonomous motivations include those that fulfill intrinsic needs (e.g., enjoyment, alignment with values, perceived importance), while controlled motivations are tied to extrinsic reasons (e.g., to avoid shame, to receive an award). Part-time $\mathrm{PhD}$ students described both autonomous and controlled motivations when explaining why they were pursuing $\mathrm{PhDs}$ and which factors contributed to their persistence.

\section{Autonomous Motivations}

In the focus groups, I asked participants two questions related to their motivations for completing PhDs: (a) why did you initially decide to pursue a $\mathrm{PhD}$ ? and (b) which factors or experiences motivate you to continue with the degree? In both discussions, findings regarding autonomous motivations broadly fell into three categories: knowledge, family, and self-fulfillment.

Knowledge. Nearly every part-time student I spoke with was also a working professional, and the majority of them spoke of pursuing the doctorate in order to investigate problems in their fields. During their professional experience, they identified 
issues within their professional practice that their current knowledge and skill sets did not allow them to properly address. In some instances, this problem was very specific and part-time students' dissertation studies were targeted on correcting the issue that initially prompted their pursuit of the PhD. For instance, one student spoke of growing up on the Navajo Nation and experiencing problems with how economic policy affected small businesses in their hometown. They described their motivation for pursuing the $\mathrm{PhD}$ as primarily wanting to help the local business owners navigate the issues associated with the policy, and therefore their motivation was largely guided by the desire to acquire the skills and knowledge needed to address the problems of their hometown.

Other students spoke of knowledge acquisition more broadly. Instead of wanting to address one issue in one place, they noticed larger systemic problems that were currently being addressed by professionals with doctorates who were operating at higher levels than their current positions. Therefore, they were pursuing the $\mathrm{PhD}$ to be able to engage in more advanced inquiries. One student in the Higher Education program explained:

Motivating me is this idea of maybe getting to a point where I can speak to the [medical doctors], and say, I don't understand what you're doing in your practice, but [I do understand] the context in which that practice is happening, and how we on the program side of it can help to ensure that we're having the best patient outcomes.

Although engaging with advanced professionals may require the credential of the $\mathrm{PhD}$, discussions about why study participants were pursuing $\mathrm{PhDs}$ were more heavily focused on the process of solving issues rather than on credentials. The focus on process was illustrated by another student in Higher Education who explained their motivation for 
doctoral study comes from "identifying a student issue [and then] digging into [the issue], researching it, finding ways to solve it, or ways to improve it or make it better [for the student]."

When discussing the relationship between the $\mathrm{PhD}$ and advancing their career goals, students tended to emphasize the research components of the degree. One student in the Social Work program explained:

[My motivation] was career-driven in terms of wanting to advance what I was doing. I'm a social worker ... I really wanted to do research. I felt like the only way for me to get the skills that I needed to be able to do that research was to pursue the $\mathrm{PhD}$.

Discussions of using research to address practice-based problems were most common among students in the social sciences (e.g., social work, public health, education). Participants in these positions spoke of using the research skills acquired through the $\mathrm{PhD}$ to advance patient or client outcomes and thus were motivated by the ability to solve issues within their respective fields.

Family. Approximately half of the part-time participants had direct caregiving responsibilities, and spoke unanimously of the importance of family in their decisions to pursue and persist in $\mathrm{PhD}$ programs. The importance of family was noted most prominently among those who identified themselves as parents but was also discussed by those who were close with their extended families.

All participants who identified themselves as parents spoke of the importance of role modeling educational success for their children, and several noted that they hoped their pursuit of the $\mathrm{PhD}$ would inspire their children to pursue advanced degrees. For example, one student described her daughter as being the "driver" behind her motivation, 
noting that she hopes to be a "a role model and some inspiration for her." Similarly, another student noted that she and her sister were both working towards graduate degrees, and this was a strong source of motivation for her: "[We're] super excited that we'll be able to share [our educational attainment] with our kids and their cousins. That means a lot to us." When students spoke of role modeling for their children, they tended to describe this as a motivation for persisting with the degree, rather than as a motivation for initially pursuing the doctorate. They described how they drew upon their desires to provide positive examples for their children when they were struggling with aspects of the programs that made persistence more difficult (e.g., feeling exhausted). In this way, family appears to be a strong source of motivation for degree persistence, particularly when persistence is met with substantial challenges, such as balancing the coursework with childcare.

Other family-related motivations were identified as contributing factors for initially pursuing the $\mathrm{PhD}$. Some students noted that they were motivated to pursue the degree in honor of family members who were unable to attain that level of education due to various life circumstances. For instance, one international student spoke of how his mother was forced to abandon her own doctoral studies due to political instability in his home country. He described his motivation as coming from a desire to complete the $\mathrm{PhD}$ to "finish what [his] mom started." Similarly, other students explained they were first generation college students and thus wanted to achieve terminal degrees as an indication of what their family can achieve and to set examples for future generations.

Although both full-time and part-time students spoke frequently of the importance of family and role-modeling in their motivation, part-time students elaborated on how their family circumstances led them to pursue $\mathrm{PhDs}$ part-time. They largely described 
their decision to attend part-time as deriving from the part-time structure allowing them to continue their careers and thereby continue to provide financially for themselves and their families. One student explained that she decided to pursue the degree part-time because it allowed her to not have "to put family life on hold while being a student because [she is] a part-time student and a staff member." By attending part-time, students are able to financially support their families in ways that may be prohibitive with the reduced salaries and benefits associated with funded full-time doctoral assistantships or fellowships.

The opportunities created by the financial benefits of enrolling in a $\mathrm{PhD}$ program part-time were particularly strong among staff members at the University. The University covers tuition for up to 6 credit hours a semester (18 credits year) for staff members, and all staff member participants were making use of this benefit to complete the $\mathrm{PhD}$ without paying tuition. While it could be argued that the enticement of free tuition is a type of controlled motivation, as it is a form of a reward for their employment, the parttime students spoke of this benefit as allowing them to continue their professional development and not reduce their annual earnings. Therefore, they saw it not as a reward but rather as an enabling factor that allowed them to achieve their goals without comprising the value they placed on supporting their families. For instance, when discussing tuition remission, one student explained that even though they have young children, they were able to pursue the PhD financially: "I'm a staff member. A big key to me being a part-time student is being able to continue with my professional development and not really stepping out of the professional world while pursuing [the $\mathrm{PhD}$ ].” Similarly, another staff member described tuition remission as being "key" to their decision to pursue the degree. 
Self-fulfilment. Although less common than career- or family-related motivations, some students spoke of their initial decision to pursue the $\mathrm{PhD}$ as stemming from their enjoyment of academic work: "I decided I wanted a $\mathrm{PhD}$ when I was in the fifth grade, which is kind of weird. I really love school. I always loved school as a kid ... So that was my initial interest in a PhD." Others described how the experience of the $\mathrm{PhD}$ aligned with various aspects of their personality, such as seeking out challenges: "I needed a new challenge. I don't know why I chose a PhD challenge ... but it was an opportunity; it was something that I knew I could do.” This type of motivation is closely aligned with intrinsic behavior, or actions performed due to an individual's interest and enjoyment.

Participants also discussed how their identities, values, and goals helped motivate them to persist with their doctoral studies. Several students identified as runners and likened their pursuit of the $\mathrm{PhD}$ to their experiences training for races:

I equate it to training for a race. The moment you cross the finish line of a half marathon is one that has stuck with me. It's like, you hate the process ... and then you cross the finish line and [get the] feeling of like everything was so worth it.

Others spoke of how they are motivated by accomplishing tasks, and how they used milestones as motivation to continue to the next phase. For example, first- and secondyear students spoke of working towards the next step (passing their comprehensive exams, defending their proposal) rather than the final step (defending their dissertation). In both cases, students are linking a specific aspect of their personality (e.g., goal oriented) with their motivation to pursue the $\mathrm{PhD}$, which suggests this motivation is coming primarily from an autonomous source. 


\section{Controlled Motivations}

When asked about their reasons for pursuing and persisting in their doctoral programs, students identified several sources of motivation that aligns with the controlled end of SDT's motivation continuum. Controlled motivations are those that are typically done for largely extrinsic reasons, such as to avoid shame, to receive a reward, or to avoid punishment (Deci \& Ryan, 1985). Findings indicate that part-time PhD students' controlled motivations fall into two primary categories: career advancement and avoidance of perceived disappointment.

Career Advancement. Nearly all participants identified career advancement as a primary source of motivation in their doctoral pursuit. In addition to the autonomous career motivations associated with knowledge acquisition, many participants explained they were motivated by the need to obtain a terminal degree to advance their careers. Several students expressed frustration that the terminal degree credential was required, as they saw it as more of a formality rather than a necessary step in their professional development. For instance, one student in a University staff job succinctly summarized the credential focus, stating that the $\mathrm{PhD}$ was "the natural next step in career progression. To move up or to open more opportunities, the $\mathrm{PhD}$ was needed." In some cases, students reported being told of the need for this credential by their direct supervisors: "my boss at the time said, 'If you want my position, you really should have a $\mathrm{PhD}$ or a terminal degree'." The need for this credential was identified by students across disciplines and was espoused by those who were planning to pursue faculty and alternative-academic (e.g., industry) positions, with one student pursuing a faculty position describing their motivation as wanting to "get letters behind my name." 
Other students were less decided on their specific career path and spoke of their motivation to pursue the $\mathrm{PhD}$ as a way to expand their career options in the future. In these discussions, it was common for participants to describe what one student labeled as "trying to find my place." In other words, several of the part-time PhD students seemed to be motivated to complete the degree because they saw the $\mathrm{PhD}$ as a way to figure out where they belong within their fields:

I'm hoping it will open some different doors for me career-wise, because I am thinking about going into faculty one day. I'm hoping that this will either tell me, yes, this is the way to go. Or it will probably be like, no, let's pursue something else. It really is a process for me to think about if this is something that I want to do.

Again, the notion that the $\mathrm{PhD}$ provides flexibility and opportunity in types of careers was noted across disciplines and career paths (i.e., faculty or alt-ac).

Closely connected to the perceived need for a terminal degree to perform advanced work is the perception that colleagues with PhDs do not respect those without them. The latter perception was particularly strong among University staff members, as participants in staff roles noted that they work primarily with faculty members who hold PhDs or other terminal degrees (e.g., MD). For example, one student who works in a staff role at the medical school of the University explained that: "In my current role, I'm just never going to get respect. [With the PhD], I feel like I'll get a little bit more and be treated as somewhat of an expert in my field. That was my initial motivation.” Another student echoed this sentiment, stating that: 
I'm smarter than these folks, but I'm not being treated with the same level of respect. And I realized I had to get these letters if I wanted to play with the big boys and girls. That's the honest motivation.

This focus on using the $\mathrm{PhD}$ as a way to gain respect professionally was typically noted as a reason for initially deciding to pursue the $\mathrm{PhD}$, and not as a reason for continued persistence. In terms of motivations to persist, students spoke frequently of not wanting to disappoint others.

Avoiding Perceived Disappointment. Findings in this section are focused on the motivations to avoid disappointing others or oneself. When asked what factors keep them motivated to persist, many students pointed to their relationships with others and not wanting to "let people down." Although the specific roles and relationships of the people that students did not want to disappoint varied (e.g., family, advisor, peers), the sentiment that there would be people disappointed in them if they were to leave the program without finishing was common across nearly all respondents. Speaking specifically of her family, one student described her motivation in this area: "I don't want to disappoint my son; I don't want him to see me start this and fail and not finish it. And my parents will be disappointed. And my husband's put all this time in with me." Similarly, another student described the idea of leaving the program as being "really embarrassing" due to how many people knew she was pursuing it, and another described how she did not want to be perceived as a quitter and "just wants to be able to say that [she's] done it."

In addition to not wanting to disappoint others, students spoke of persisting with their degrees because they did not want to disappoint themselves. This discussion most frequently occurred in students describing the sunk costs associated with their PhDs. In particular, students who were beyond the first year in their program spoke of continuing 
with their programs due to the time and energy that they have already spent working towards their degrees:

The thing that motivates me really is thinking about the time and effort and money I have already put into it. Like, okay, great, I have tuition remission, but you still have to pay the fees; you still have to pay for your textbooks. Thinking about how I gave up going to see the Lion King with my niece because I had class, and all those things build up. If I don't finish, then all that was for nothing.

One student reflected on the issues with this type of motivation, noting that it is only enough to make her do the bare minimum amount of work required; she struggles finding motivation to do anything beyond this level of work. Students also identified other factors that make it difficult for them to find motivation to persist.

\section{Demotivational Factors}

This theme derives primarily from responses to the request to name factors or experiences that caused participants to lose motivation. Although these responses were grounded in participants' specific circumstances (e.g., childcare responsibilities, conflicts with their advisor), causes of reduced motivation were generally attributed to feeling isolated, exhausted, or overwhelmed.

Feeling Isolated. Isolation was an issue identified by all doctoral students, regardless of enrollment status, discipline, or doctoral phase. However, there were variances in how students viewed their isolation based on their doctoral stage. For instance, in the dissertation stage, they spoke of a lack of connection to their peers once they finished coursework:

[The] dissertation is a very lonely process because it's just you with your dissertation ... when you're doing classes, you sort of have that cohort and 
support group that gives you that motivation, because you're seeing them on a constant basis, and you're interacting with them. And then once you get done with classes, that motivational piece sort of goes away ... and you have to find that self-motivation.

While isolation caused by the dissertation process was noted frequently by both full- and part-time students, part-time students also spoke of feeling disconnected from their peers and faculty in their programs in other phases of their doctoral program, such as coursework and comprehensive exams.

Part-time students typically attributed the cause of these feelings of isolation and disconnection to "feeling like a square peg in a round hole." That is, part-time students expressed feeling as if they were the only student in their classes who was not following the traditional $\mathrm{PhD}$ model of enrolling full-time and having an assistantship. For example, one part-time student described her coursework experience:

I felt so alone, so often. I would see other people being on three research projects, and taking nine credit hours a semester. And I was like, oh my God, I'm seven months pregnant and may have the baby in this class. I felt just so alone all the time.

With full-time employment and childcare responsibilities, this student explained that she was unable to relate to the experiences of her full-time peers, and this lack of connection caused her to feel isolated even when she was surrounded by them. Similarly, another student expressed how aspects of the doctoral program designed as preparation for faculty careers (e.g., publishing) left her feeling alienated from their peers:

Since I'm part-time and not necessarily looking for a full-time faculty position ... I think it was pretty alienating to me when all the conversation and discussion was 
about publishing and presenting. And that's just not where I am; maybe I should be a little bit more. But that was pretty alienating for me, and so I felt a little on an island.

Although this student recognized that publishing and presenting were fundamental to the $\mathrm{PhD}$ experience, she also explained that her part-time status frequently prevented her from doing more than the bare minimum required, and therefore she did not feel like she had the time to take on the extra work associated with publishing and presenting at conferences. Many students described how it felt as if they were the only student with these feelings, despite the high percentage of part-time students enrolled in many of the degree programs.

Adding to the issue of isolation is a lack of connection that many part-time students feel with their advisors. Although part-time students were divided on this topic, as some students noted that their advisors were one of their primary supports and contributed substantially to their motivation to persist, those who did not have a strong advisor relationship spoke of how this lack of relationship negatively affected their sense of belonging and their motivation:

I've got like, nothing. My advisor is not reaching out to me. No one's checking in ... And I know my advisor wants me to finish but she's always like, 'this is you driving [your progress]. You let me know what you need.' Well, I need her to be like, 'Where are you? What's going on?' And there's been none of that.

Another student expressed feeling as if they were not a priority for their advisor: "You're 10th on [your advisor's] priority list. They just want you to turn in the paper, and then they'll get to you when they get to you." This student perceived that they were not a priority for their advisor due specifically to their part-time status. This feeling was echoed 
by other study participants, and suggests that weak advisor-advisee relationships contribute to feelings of isolation and are thus a source of demotivation for part-time students.

Feeling Exhausted. Unlike feelings of isolation, which were common among full-time and part-time students, feelings of exhaustion were far more prevalent among part-time students. Part-time student participants were almost universally balancing their $\mathrm{PhD}$ programs will full-time employment, and many of them also had caregiving responsibilities. Nearly all of the part-time students spoke of struggling to find motivation due to exhaustion at some point during their doctoral program. For instance, one firstsemester part-time student explained how exhausting it was to work a full day and then attend night class:

When I feel the least motivated ... is right after a very draining class. I'm getting a lot out of it ... But you know, I get to the office at eight o'clock in the morning. I'm here for eight and a half hours, and then two and a half hours at class. When it's said and done, I've been sitting at this desk for 12 hours, and it's just like, what am I doing? I'm exhausted.

This student noted that COVID-19 was an exacerbating factor in their exhaustion, as they were attending class online and therefore sitting in the same seat for the duration of their work and school day. Another student who worked in a student affairs staff position spoke of how COVID-19 had caused a substantial increase in their workload, and this increase has made it more difficult for them to complete their doctoral work: "It's just mentally exhausting. And so that really just makes any motivation I have to do anything else go out the window." 
Although part-time $\mathrm{PhD}$ students frequently mentioned COVID-19 as a contributing factor to their current exhaustion, others noted that causes of their exhaustion existed before the pandemic:

I do work full-time, but I decided to take three classes. And I will say that it truly wore me out. After I got done with the spring, I was just literally like, I'm tired. I don't want to do anything ... I think I'm like a superwoman ... I should be like writing a little bit or reading more, but I think I was truly just not motivated to do it. Because the spring semester had truly just worn me out.

Another student also described feelings of guilt associated with exhaustion. They explained that when they were a master's student and a full-time employee, they were able to not do extra work on the nights that they had class. However, they believed their PhD program necessitated hours of work every night, regardless of other circumstances: I would just assume, okay, I have class Tuesday, Wednesday; those are just nights I [don't do additional work]. But now with the amount of work that I think is expected [in the $\mathrm{PhD}$ ], I don't know if I can say [the hours after night class are] the time that I just discount. I don't know if I can say that anymore. And the thought of that kind of exhausts me.

This student spoke of feeling as if they were never doing enough, despite working long hours every day. All participants in this focus group session agreed with this feeling, indicating that many part-time students may feel their efforts are not meeting the demands of their degrees.

Feeling Overwhelmed. Unlike isolation and exhaustion, the feeling of being overwhelmed was attributed not to being different or feeling worn out, but rather feeling split between competing responsibilities and priorities. Part-time $\mathrm{PhD}$ students largely 
described their decision to enroll on a part-time basis as being borne out of a financial necessity, as they explained they could not support themselves or their families on the reduced salary of an assistantship. The need to maintain a full-time position contributed to the students experiencing difficulties with prioritizing their doctoral work over their professional careers. Students largely spoke of wishing they could spend more time focusing on their doctoral studies, yet the reality of their situations necessitated they prioritize their careers. For instance, one student explained how their increased workload from COVID-19 caused them to reduce the number of classes they were taking and therefore slow down their overall time-to-degree:

That's the hard part. I dropped one of my classes that I was taking this semester, and that pushed back finishing my comps. And I'm like, you know what, I don't even care at this point. I'm doing it because I have to.

Other students described how their attempts to balance competing responsibilities led to decreased performance in their careers or doctoral programs. When describing the impacts on her career, one student explained:

I took three classes at once, and it was during [a busy time of year at my job]. [It was the] worst decision I probably ever made. Because I really couldn't give the attention that was needed to my [job] then ... I put a lot of stress on myself. I'm usually good at hiding it, but people around me noticed the tiredness and everything that I was going through.

Although part-time students spoke of prioritizing their careers above their doctoral programs, they also described the guilt and stress that came along with these decisions. The negative feelings associated with these choices suggests that, if given the choice, 
many part-time students would prioritize their doctoral education over their current professional positions.

Similarly, those students with young children described the difficulties that came from trying to balance their $\mathrm{PhD}$ work with their caregiving responsibilities. As with career prioritization, many of these students spoke of having to choose between what their children need and what needs to be done for their doctoral programs:

But the time away from my kids is hard ... To be honest, I have started missing class, like send an email that I can't be there. I've got to do this for my girls, because that's not going to be here the next time. You know, I need to do those things. It's a balancing act, and I get that, but I can't let those moments pass either. Again, there was substantial guilt and stress associated with decisions to prioritize their doctoral work over spending time with family. As another student described, "the mom guilt is real in this because you know how much time you're taking away from your children. It's tough." Students recognized that being successful in their doctoral programs necessitated substantial commitments of time and effort, but they struggled with finding this time in their otherwise demanding schedules. Frequently, students spoke of having to compromise the quality of the work they do in their doctoral programs due to this stress: "I just am so overwhelmed with the bare minimum of what I have to do." So while parttime students may be driven by motivation related to supporting their families or advancing their careers, these same factors can also be a substantial source of stress that decreases motivation overall.

\section{Satisfaction}

The quantitative analysis showed a negative relationship between satisfaction and controlled motivation (i.e., controlled motivations lead to a decrease in overall 
satisfaction). Yet in the focus groups, part-time students were reluctant to describe themselves as unsatisfied, regardless of how they described their motivation. That is, part-time students would describe numerous issues with their doctoral program that they were unsatisfied with (e.g., advisor, course work), but then describe themselves as satisfied overall. Full-time students were far more likely to identify as being unsatisfied and to name specific programmatic factors that led to their dissatisfaction. However, when part-time students were asked to describe their satisfaction, many of them discussed issues with their programs or personal circumstances that detract from their overall satisfaction, indicating that they may be more dissatisfied than they choose to admit.

Results of first and second cycle coding further support this finding. For example,

first round coding involved identifying sources of satisfaction and dissatisfaction broadly, and resulted in 15 codes for sources of satisfaction and 89 codes for sources of dissatisfaction. Thus, findings suggest that even when indicating satisfaction, part-time students are wrestling with a range of challenges and issues that may cause them to experience feelings of dissatisfaction. Therefore, although part-time students are likely dissatisfied with many aspects of their doctoral programs, they describe themselves as primarily satisfied with the overall experience.

\section{Factors that Contribute to Satisfaction}

Those students who spoke of being highly satisfied with their doctoral experience were most likely to identify sources of support that contributed to this satisfaction. These sources of support came from peers, faculty, and personal growth.

Peer Support. One student in particular is part of a cohort of students who are all staff members, as well as women of color, and who are all pursuing their $\mathrm{PhDs}$ in the same program part-time. When speaking of this cohort, she said that it "truly contributes 
to my satisfaction of the program, because I'm on this journey with four other people. And I think that has truly been a blessing for me and truly contributed to that satisfaction." Despite the satisfaction that this student gained from this experience, parttime cohorts appear to be extremely rare across disciplines, as she was the only part-time participant who indicated she had a close cohort to rely on for support.

Faculty Support. Other students described the effects of their advisors and mentors contributing to their overall satisfaction, although generally strong advisor or mentor relationships were rare among part-time student participants. Those who did maintain strong relationships described how their advisors have helped them navigate the challenges of part-time study. For instance, one part-time student explained that his advisor has helped him prioritize competing demands within the program: "her perspective has been helpful for me to identify areas where I need to be more selfmotivated, or more aggressive, and what I need to get done.” Aside from advisors, informal mentors also appear to be a strong source of support for students who form these relationships. For instance, a student who moved out-of-state to another institution for work described connecting with a faculty mentor at their new institution and explained that this mentor has helped guide their work:

I've been extremely happy with my mentor and how receptive she's been. We have weekly calls where we talk about what I'm doing and how things are going. And so I felt a lot more supported in that process with her than I ever felt the three years that I was taking courses.

Although rare, having strong faculty relationships with frequent touch points appears to be a strong indicator of overall satisfaction in part-time students, regardless of whether these relationships develop between advisor and advisee or mentor and mentee. 
Personal Growth. When discussing which factors led to their overall feeling of satisfaction, part-time students also pointed to their demonstrated growth in academic and professional abilities. Students reported feeling better prepared to engage with scholarly articles and conduct research of their own, and several students discussed how these improved skills have benefited them in their professional lives. One part-time student in particular spoke repeatedly about the importance of skill development and knowledge acquisition in her PhD satisfaction. Although she was one of the students who described her motivation as being primarily credential-focused (i.e., needing the degree for promotion purposes), it was not career progression that contributed to her satisfaction: One of my first sources of satisfaction ... was learning how to read a scholarly article. I was really surprised [because of] how I remembered looking at these documents for years. But now I understand and know how to actually go through it. And also, what makes me satisfied is when I can teach others. I can work with my students now and know how to analyze the abstract and how the study went ... and when I feel confident in what I can do to teach others I'm satisfied.

Rather than being satisfied through her career progression, she explained that her satisfaction derived most fully through her application of skills that she learned through the program. In this way, her satisfaction was coming from an internal source (i.e., confidence in her abilities) rather than an external reward. Similarly, another student spoke of pursuing the $\mathrm{PhD}$ due to the rigor associated with the degree, as they had completed a professional doctorate in the past and felt that that degree had not been challenging enough; they gained satisfaction from being challenged by the $\mathrm{PhD}$. The experiences of both students suggest that although part-time students may engage with 
doctoral programs in non-traditional ways, traditional aspects of the degrees (knowledge development, academic rigor) are sources of satisfaction.

\section{Factors that Detract from Satisfaction}

Although students were unlikely to label themselves as "not satisfied," they spoke extensively of perceived problems with their programs and institution that detracted from their satisfaction. While some of these problems have been exacerbated by the COVID19 pandemic, the majority of them have been longstanding according to participants. The students who described perceived problems varied across disciplines, doctoral stages, and personal characteristics. Sources of dissatisfaction centered around assumptions that their programs were designed for full-time students and little, if any, accommodations had been made for part-time students. The lack of part-time student support was identified as coming from faculty, programs, and broad student service areas within the University (e.g., the Graduate School, the library, the writing center).

Perceived Shortcomings of Faculty Support. The most common finding in this area was a widespread belief that program faculty considered part-time students to be lesser than their full-time peers. This perception came most directly from interactions with faculty members, as many students expressed feeling as if faculty were disinterested in working with part-time students or did not take part-time students seriously when compared to full-time students. In some instances, part-time students described feeling as though faculty did not understand the demands on their time:

Sometimes I can't be in class tonight, because I have a work commitment that I have to attend. I think a lot of the faculty members need to realize that not everybody has a TA or a GA, but some of us are working full-time jobs. And sometimes we just need some grace and patience. 
The perception that faculty assumed all students were full-time was largely held by parttime students across disciplines, contributing to the finding that these students widely feel as if they are outsiders within their programs. For example, other students described the experience of being a part-time $\mathrm{PhD}$ student as feeling like "an afterthought" or a "second class citizen."

Part-time students largely assumed this feeling of differential treatment compared to full-time students was due to their own lack of adherence to the traditional PhD student model. They described believing that faculty expected all $\mathrm{PhD}$ students to follow traditional models of doctoral education. In other words, part-time students expressed beliefs that program faculty assumed all doctoral students were enrolled full-time, held assistantships, spent their time focused on completing research in order to publish and present at conferences, and held the ultimate goal of attaining tenure-track faculty positions. Although many part-time students described their interest in improving their research skills and advancing knowledge in their fields, few of them described needing these skills in order to publish or present at conferences, indicating that part-time students may be more interested in the practical application of knowledge than the creation and dissemination of knowledge in traditional academic avenues (e.g., conferences, journals).

While some students admitted that they were wholly disinterested in publishing and presenting at conferences, it was more common for students to describe their inability to add additional work to their lives. One student explained that although they wished they were able to be more involved in research and publishing, they felt that adding to their workload was "just not realistic." Students also expressed frustration with faculty's perceived focus on preparing for tenure-track positions, noting that the ever-declining job market is creating a reality in which "those positions may not exist." Perceptions of 
differential treatment also led to frustrations among many part-time students who were acutely aware that their tuition paid faculty salaries: "I'm still funding this department; I'm still valid here. I felt like we were being treated poorly [by faculty] for [our part-time status, which] we have no control over." Feelings of inequity seemed to be exacerbated by the majority of part-time students expressing that their part-time enrollment was a necessity rather than a choice. Several students remarked that if their life circumstances had been different and they were able to choose between full- and part-time enrollment, they would have pursued the PhD full-time.

Perceived Shortcomings in Program Supports. Aside from faculty relationships, students described their programs as detracting from their satisfaction due to the perceptions that programs were designed for and focused on supporting full-time students only. For example, the majority of part-time students did not have an established cohort to rely upon for support and advice during their program. Students without cohort support spoke of its negative impact on their overall satisfaction level, saying that having "no cohort has been very tough." Students were largely aware of how cohorts can contribute to the positive experiences of full-time students, and this awareness seemed to heighten their feelings of missing out on this aspect of the doctoral experience:

Whenever I go to big conferences, people are connecting with their cohort and connecting with people that were in higher levels of previous cohorts, and they have those connections for years and years. I don't feel like that's going to happen [for me].

Beyond networking and associated benefits, students expressed a desire for the shared experiences and connections that can come through cohort models: 
I wish that there had been more of an opportunity to kind of sit around and be like, oh, I'm not in this alone. Like everyone else who is in this process and going through their journey is having hiccups and hurdles, and they may not be mine.

But you know, they're not perfectly sailing through this either. Like they're having their moments as well.

Students largely described the lack of cohort as contributing to their feelings of isolation and their overall lack of belonging in their programs. In many ways, part-time students seemed to crave connections with other part-time students who understood their doctoral experience. Several students noted how the focus groups, which brought together parttime students to discuss their shared experiences, "felt like therapy."

Beyond cohort supports and faculty relationships, part-time students also expressed feeling as if their programs did not consider their needs when crafting policy or designing programmatic materials. For instance, one student explained that in their first advising session, they were given a full-time program plan, despite their advisor knowing that they were attending on a part-time basis. Program plans are given to students in order to help guide them through their coursework and successfully complete their requirements within a timeframe, but the structure of the program plan will differ substantially based on enrollment status. This student expressed frustration with being given incorrect materials that did not aid in their degree completion.

Perceived Shortcomings in University Supports. Many of the part-time students were also employed in university staff positions, and several of them worked in student affairs positions. Perhaps for these reasons, many students were well informed about the types of supports that various university units (e.g., the Graduate School, the library) offer for doctoral students (e.g., invited talks, workshops, student socials). Yet 
they described believing that part-time student needs were not considered when designing these supports, as they were primarily offered during working hours when part-time students are typically at work and therefore unable to participate. One student affairs professional reflected on this feeling of being unsupported by the institution by explaining how her experience as a part-time student made her better able to understand the feelings of other underserved student populations:

[Being a part-time $\mathrm{PhD}$ student] is kind of what it feels like to be a student on a college campus that doesn't serve you. I had never really had that experience before, like many students do throughout their [education]. But I was finally like, oh, I'm not the target here. Okay, that's clear. I just wish there was more support. Being unable to participate in doctoral student programming contributed to the overall feeling described by part-time students that they were not the primary concern among faculty, their programs, or their universities.

Beyond student supports, part-time students also described feeling as if the culture of the University did not value them in the same way it valued full-time students. For instance, one first-year part-time student described an experience they had at the collegewide doctoral student orientation:

At orientation something was said to the effect of 'will [full-time students] have opportunities for engaging with faculty and university stakeholders that part-time students will miss out on?' And the answer was 'YES!' ... That was kind of frustrating, because I feel like I have all kinds of opportunities as a part-time $\mathrm{PhD}$ student. It's one of those moments where you see yourself a certain way, and then you realize other people see you a different way. Like, I don't feel limited as a part-time PhD student, in terms of what I can do, or partnerships I can create, or 
the projects I can work on, or the relationships I can build. So, to have a faculty member say that was kind of demotivating.

Orientation is supposed to be the first time that students engage with their college at the doctoral level, and thus the inclusion of material that specifically alienates part-time students may be contributing to the overall assumption among these students that they are less valued than those who attend full-time.

\section{Summary}

This chapter provided findings from a survey $(N=430)$ and focus groups $(N=50)$ with doctoral students at one university regarding their motivation and satisfaction. Findings addressed three research questions: (a) Does motivation for doctoral studies influence overall doctoral program satisfaction for part-time PhD students? (b) How do part-time $\mathrm{PhD}$ students describe their motivation for persisting and pursuing in their doctoral programs? (c) How do part-time $\mathrm{PhD}$ students describe their overall satisfaction with their doctoral programs?

Findings suggest that part-time $\mathrm{PhD}$ students share much in common with fulltime students in terms of motivation and satisfaction, as enrollment was not a significant predictor of satisfaction. The decision to enroll on a part-time basis is typically dictated by personal circumstances and desire for career advancement. Once enrolled, part-time $\mathrm{PhD}$ students are motivated to persist by knowledge advancement and relationships with others, yet access to these relationships is restricted due to their part-time status. Sources of demotivation are consistent with those of full-time students and include feeling isolated, exhausted, and overwhelmed; circumstances associated with part-time study exacerbate these feelings. Like motivation, satisfaction is primarily derived through relationships with others, feeling supported, and personal and professional growth. Yet, 
also like motivation, part-time enrollment limits access to many of the relationships and resources that provide satisfaction. An overall lack of community support and access contributes to part-time students' perceptions that they are valued less by their programs, faculty, and their institution. In the following chapter, I discuss these findings in relation to the literature about doctoral education and provide implications for research and practice. 


\section{DISCUSSION}

There were two purposes to this study: (a) investigate the relationship between motivation and satisfaction among part-time $\mathrm{PhD}$ students, and (b) build knowledge about the part-time $\mathrm{PhD}$ student experience. In this chapter, I discuss major findings from the study as they relate to the literature about doctoral education, part-time $\mathrm{PhD}$ student experiences, and the guiding theoretical framework of Self-Determination Theory (Deci \& Ryan, 1985). Through this discussion, I show how the study answered the three research questions:

1. Does motivation for doctoral studies influence overall doctoral program satisfaction for part-time $\mathrm{PhD}$ students?

2. How do part-time $\mathrm{PhD}$ students describe their motivation for persisting and pursuing in their doctoral programs?

3. How do part-time $\mathrm{PhD}$ students describe their overall satisfaction with their doctoral programs?

Findings from the quantitative portion of the study indicate that SDT explains the relationship between motivation (autonomous, controlled) and satisfaction for this sample regardless of enrollment status, with autonomous motivation leading to increased satisfaction and controlled motivation leading to decreased satisfaction. Quantitative

findings further indicated that full-time students report higher levels of both autonomous and controlled motivation, while part-time students report higher levels of satisfaction. 
However, qualitative findings indicate that these reported levels of satisfaction may not be fully accurate, as part-time students reported substantial and frequent causes of dissatisfaction with their PhD experiences.

The qualitative phase provided further nuance to the quantitative findings. Qualitative findings indicate that part-time students derive autonomous motivation from gaining new knowledge, their families, and fulfilling their personal goals. At the same time, they are motivated by factors on the controlled end of the motivation spectrum, such as advancing their careers and not disappointing people in their lives. Factors that detract from part-time student motivation are primarily related to feelings of isolation, exhaustion, and being overwhelmed. Part-time students described deriving satisfaction from personal growth and sources of support (e.g., advisors, cohorts), but more commonly were dissatisfied with the supports they received from program faculty. Similarly, they frequently described feeling as if program policies and institutional supports were not designed for part-time students. All of these perceptions likely contribute to many part-time students feeling alienated and alone in their doctoral pursuits.

Although many of these findings overlap with experiences of full-time students as described in the literature (e.g., Mason, 2012; Barnes \& Randall, 2010; Zhao et al., 2007), part-time students described how the circumstances of their enrollment exacerbated many of these issues. For instance, although all doctoral students likely struggle with feeling exhausted or overwhelmed, part-time students situated these conversations in relation to completing doctoral requirements while also maintaining a full-time career (that often must take priority); maintaining the balance between PhD work and a full-time career is not typically an aspect of the full-time PhD student experience. Other issues identified by 
the part-time students, such as perceptions of unsupportive faculty or lack of peer connections, appear to be specific to the part-time student experience.

\section{Interpretation of the Findings}

Findings from this study suggest that the relationship between motivation and satisfaction in part-time PhD students is comprised of three related themes: (a) community motivates part-time students, but access to community may be limited; (b) untested assumptions about the $\mathrm{PhD}$ and the part-time student experience detract from overall satisfaction; and (c) part-time student career motivation is both intrinsic and extrinsic. Overall, study findings indicate that part-time $\mathrm{PhD}$ student motivation is similar to that of full-time students enrolled in doctoral programs at the research site, yet satisfaction is affected by the reduced exposure and perceived misunderstandings typically associated with part-time enrollment.

\section{Community}

Echoing previous studies about the role of community in doctoral education (Baker \& Pifer, 2011; Gardner \& Gopaul, 2012; Lovitts, 2011), my findings indicate that community support is a critical factor in motivating doctoral student persistence regardless of enrollment status. The need for community is supported by SDT, which posits that relatedness with others is an innate need that drives human behavior (Deci \& Ryan, 1985). While many studies look at doctoral student communities from a programmatic lens (e.g., faculty, student peers), Baker and Pifer (2011) expand the definition of doctoral community to also include family, friends, and professional colleagues. In my study, part-time students spoke of drawing upon all of these sources for both logistical (e.g., childcare) and emotional support. Students who felt well supported were likely to identify specific individuals in their lives (partners, friends) that were 
enabling them to be successful in their doctoral programs. As a whole, my findings adhered to literature about the role of relationships and support in doctoral student success and extend that literature by demonstrating this need among part-time $\mathrm{PhD}$ students.

Yet while all doctoral students seem to benefit from community support, the ability to access and integrate into communities may be considerably affected by parttime enrollment. Part-time students described how their full-time employment affected their doctoral student experience, as full-time employment restricts part-time students from being able to integrate into their doctoral communities. Most simply, the time commitment inherent in full-time work prevents students from participating in extracurricular activities, such as invited talks, workshops, or student socials. Participants explained that they are constantly rushing between commitments and therefore do not have the ability to take on any activities beyond what is minimally required of their programs.

Practically, this lack of time prevents students from spending time with peers and program faculty. While many doctoral students may create relationships with their peers during extracurricular or social events, part-time students' inability to participate inherently leads to their exclusion from relationship-building. This finding echo that of Deem and Brehony (2000), who found that full-time students have greater access to program faculty and advisors due to their physical presence (e.g., working in GA offices, arriving to class early). The participants in my study frequently noted feeling detached from their advisors and professors due to their infrequent meetings and rushed interactions. In this way, the findings from my study also mirror those of Neumann and 
Rodwell (2009) who found that part-time students are less integrated into academic and peer cultures than full-time students.

Compounding the logistical challenges associated with full-time enrollment, parttime students may also feel a lack of belonging even when they are physically present (e.g., in class, meeting with faculty). Participants described how their differing doctoral experience left them feeling alienated from peers who were heavily involved in traditional academic work (e.g., assisting faculty with research, presenting at conferences). While Baker and Pifer (2011) noted that feelings of isolation may be common among doctoral students in the dissertation phase, my findings indicate that for part-time students, this feeling of isolation can occur at any point during the doctoral journey, but may be stronger during the coursework component. Because part-time students are not physically present at many of the opportunities that are created for networking and relationship building, they may not have the opportunity to form relationships with other part-time students and are thus left feeling as if they are the only student experiencing the challenges of part-time study.

Part-time students nearly universally expressed feeling isolated from their (presumptively full-time) peers. However, while some participants were the only parttime student in their programs as a matter of policy (i.e., the program only admits one part-time student per year), the majority of participants were in programs that appeared to have substantial part-time enrollment. The discrepancy between the number of part-time students in a program and the perceived isolation of the students indicates that part-time students are not building relationships with each other and thus not experiencing the benefits of peer support in their doctoral programs. The isolation from peers reported by nearly all part-time participants stands in stark contrast to the one part-time student who 
was a member of a part-time specific student cohort and who spoke at length about the benefits of the cohort to her experience.

Compounding the feeling of isolation is a perceived lack of external resources (e.g., how-to books, blogs, online communities) available to part-time students. Although there are numerous external resources available to full-time doctoral students, resources tailored to part-time students are scarce. Nearly ten years ago, Gardner and Gopaul (2012) found that part-time doctoral students felt as if there were no guides or resources on how to be successful as a part-time $\mathrm{PhD}$ student, and my findings indicate that this lack of resources persists today.

Ultimately, my findings indicate part-time students are lacking access to community physically, psychologically, and intellectually. Lacking community support has been shown to lead to numerous negative outcomes for doctoral students, including reduced persistence (Lovitts, 2001), decreased satisfaction (Zhao et al., 2007; Deem \& Brehony, 2000), and decreased mental well-being (Muszynski, 1988). Adding to these negative effects, reduced access to academic and peer communities also prevents parttime $\mathrm{PhD}$ students from having their assumptions about faculty beliefs and programmatic designs tested. My findings indicate that these untested assumptions detract considerably from overall satisfaction.

\section{Assumptions}

While it is likely that all doctoral students enter their programs with preconceived notions about what their experience will be, most students have these assumptions tested and altered through doctoral student socialization. Socialization, or the process through which doctoral students learn the conventions of their programs and disciplines on the journey toward becoming independent scholars, occurs largely through interactions with 
faculty, advisors, and peers (Weidman et al., 2001). Such interactions require time and presence, and my findings indicate that part-time $\mathrm{PhD}$ students have substantial restrictions on both aspects of their doctoral experience. Dressel and Mayhew (1974) were suspicious of the discrepancy between time needed for socialization and time available in part-time enrollment, arguing that it was unlikely for part-time students to achieve proper scholarly or professional socialization with the reduced amount of time they spend with faculty and peers. My findings support Dressel and Mayhew's concern, as I found that many part-time $\mathrm{PhD}$ students held assumptions about themselves and their programs that may have been altered during the socialization process.

Without this socialization, it is likely that part-time students are experiencing feelings of otherness, and such feelings may contribute to their assumptions about their own experiences remaining untested. Pifer and Baker (2014) found that otherness, or "feelings of negative distinction, isolation, or lack of fit within a given social context based on one or more aspects of one's identity in relation to other group members and group norms" (p. 15), can substantially shape a doctoral student's experience, their perception of the experience, and their subsequent belief in their own potential for success and satisfaction within their doctoral programs and future careers. In other words, feeling like an outsider, which many part-time participants described, can shape the doctoral journey and the relationships within it, and thus may contribute to the finding that study participants' assumptions appear to be present at all stages of the doctoral journey) and therefore untested. The consequences of these untested assumptions are a lack of professional growth, a misunderstanding of the role of the self and faculty members in the doctoral process, a reduced sense of belonging, and negative perceptions about their $\mathrm{PhD}$ experience. 
Evidence of untested assumptions was perhaps most apparent in the widely held belief that program faculty do not value part-time students to the same degree that they value full-time students. This belief may stem from a perception of difference in values between faculty members and part-time students who are not seeking academic careers. As Pifer and Baker (2014) explain, students who perceive themselves as different from faculty members may also feel that they are excluded from the benefits of faculty relationships, including support, mentoring, access to academic and professional opportunities, and a sense of belonging with their departments. Gardner and Gopaul (2012) also found this belief in their study, where part-time participants described feeling minoritized and inferior to full-time students.

In my study, the belief that faculty saw part-time students as less than full-time students was expressed by the majority of part-time participants, regardless of program discipline or doctoral stage, and it was typically attributed to a belief in faculty prioritizing self-replication. In other words, part-time students largely described believing that faculty were primarily (if not exclusively) concerned with doctoral students who mirrored their own experiences (e.g., attended full-time, received an assistantship or fellowship, published and presented research in order to prepare for future tenure-track faculty positions).

While there may be faculty members who hold prejudices against part-time students, untested assumptions of this nature are likely to be the result of a lack of socialization to faculty roles broadly and individual program faculty specifically. For example, part-time students described beliefs that they were low on their advisors' priority lists and had to frequently wait extended periods of time for feedback on paper drafts. Participants attributed this lack of attention to their part-time status. However, 
when taken into consideration alongside the realities of increased faculty workloads and a devaluing of advising and mentoring in faculty evaluations (Miller \& Seldin, 2014), it becomes apparent that delays in responses may be due to factors wholly external to the student's enrollment status.

Regardless of enrollment status, many doctoral students may begin their programs with a misunderstanding of faculty roles and responsibilities (Weidman et al., 2001). Typically, students would learn about the varied aspects of faculty careers through the socialization process, as this process is considered the first step in preparing future faculty members (Sweitzer, 2009), and my findings indicate that part-time students may not be experiencing this socialization. Compounding this issue, Pifer and Baker (2014) found that doctoral students who do not intend to pursue faculty careers experience feelings of professional otherness. In their study, these feelings led to two related outcomes: "students judged themselves harshly or they judged their departments negatively" (p. 21). In other words, a perceived disconnect between a student's career goals and traditional academic careers can shape the student's PhD experience in negative ways.

Although Pifer and Baker's (2014) study focused solely on full-time students, my findings indicate that a perceived disconnect in career goals and values also negatively affects part-time students. In addition to attributing negative feelings to themselves and their departments, students in my study assigned these negative feelings to their enrollment status. The belief that faculty are dismissing part-time students because of their enrollment status was a common explanation for decreased satisfaction, and therefore increasing satisfaction among this student population needs to address misconceptions in faculty roles and responsibilities. Similarly, my findings indicate that 
some part-time students are misunderstanding the purpose and value of the $\mathrm{PhD}$, and their assumptions about the degree are also detracting from their overall satisfaction. When discussing perceptions of faculty interactions, students frequently explained they were experiencing differential treatment due not only to their enrollment status, but also to their lack of interest in research. I heard repeatedly from part-time participants that they had no interest in research or the associated tasks of publishing and presenting. While the latter tasks of publishing and presenting are arguably the result of academic culture and pressures associated with academic careers, the $\mathrm{PhD}$ is fundamentally and unquestionably a research doctorate. Regardless of a student's interests or career intentions, the emphasis on research training is a critical and unifying theme that spans disciplinary boundaries and has remained constant for hundreds of years (Radford, 2001). In other words, a students' intention to use the $\mathrm{PhD}$ to advance in a non-research focused career does not negate the purpose of the degree, nor should it dictate the curriculum they are taught.

For some part-time students, there seems to be a common assumption that the purpose of pursuing a $\mathrm{PhD}$ is to attain the "letters behind [one's] name" rather than the skills and abilities associated with advanced research training. Several participants told me stories of how this assumption was informed by direct supervisors or mentors who told them that they needed the terminal degree in order to advance from their current roles. Although many part-time participants expressed an interest in research, the participants that had been explicitly told to pursue the degree for professional advancement purposes by supervisors or mentors universally expressed a disinterest in research. Perhaps more importantly, none of these participants seemed to possess scholarly identities, as they all noted they were in the program exclusively for the credential and were only concerned with completing bare minimum requirements in order 
to graduate. Deem and Brehony (2000) found that developing a scholarly identity necessitates exposure to research cultures, and Baker and Lattuca (2010) echoed this finding, showing how socialization is a key factor in scholarly identity development.

Seen through this work on scholarly identity development, the tendency of these part-time participants to disregard the research aspects of the $\mathrm{PhD}$ due to an assumption that they only need the credential may come from a lack of socialization into the research cultures of their disciplines. Furthermore, this assumption reflects a lack of socialization into the field, as the students seemed largely unaware of how the skills and knowledge they were gaining in pursuit of the PhD might contribute to their overall abilities to be successful in their careers, which were all in fields closely related to their doctoral programs. They seemed to assume that the requirement of a terminal degree was a technical formality and not indicative of the advanced abilities and knowledge required of individuals in the positions they were seeking. In short, their assumptions about the degree reflect a fundamental misunderstanding of the PhD's theoretical purpose, its practical use, and its relationship to their own field specific professional goals. However, it should be noted that participants focused on credential attainment over skill development were a substantial minority in my study participants, and the majority of participants expressed a nuanced career driven motivation.

\section{Career Motivation and Preparation}

Although my quantitative phase indicated that many of the extrinsic aspects of career motivation (e.g., seeking a better salary, leaving an undesirable job) are negatively associated with satisfaction, the qualitative findings suggest that career motivation in part-time PhD students contains both extrinsic and intrinsic elements. I found that career motivation among part-time $\mathrm{PhD}$ students is frequently derived from a desire to grow 
knowledge and solve problems in one's field of study. According to SDT, this type of motivation is closely aligned with autonomous motivation and identified regulatory behavior, which focuses on performing an activity due to the belief in its importance (Deci \& Ryan, 1985). Just as full-time PhD students express what Gardner (2008) has called "intensive devotion" to their field of study (p. 36), part-time participants in this study expressed strong commitments to their disciplines that are reflective of autonomous motivation.

That said, there does appear to be a relationship between career driven motivation and satisfaction. Sakurai et al. (2017) found that career motivation is a significant predictor of satisfaction in doctoral students, with students who were motivated by developing their career prospects reporting higher levels of satisfaction. In some ways, my quantitative findings contradict the findings of Sakurai et al., as my findings shows a significant negative relationship between career motivation and satisfaction. However, their study took a more comprehensive understanding of the intrinsic and extrinsic aspects of career motivation (e.g., connection to research interests, expanding career possibilities), while my quantitative instrument only contained extrinsic career items (e.g., pay increase, promotion). In this way, my qualitative findings, which suggest that doctoral career motivation is both intrinsic and extrinsic, support their findings.

However, my findings also suggest that the relationship between career motivation and satisfaction may differ for part-time students. Sakurai et al. (2017) were studying full-time doctoral students, and although they did not inquire about career plans of these students, it is reasonable to assume that many of them were planning on pursuing traditional faculty appointments given that faculty career preparation is the longstanding primary purpose of the full-time $\mathrm{PhD}$. Therefore, the participants in Sakurai's et al. 
(2017) may have been deriving satisfaction from the alignment between their doctoral programs and the preparation for their future careers (e.g., training on publishing, presenting). Yet as Pifer and Baker (2014) found, doctoral students who experience otherness due to having career goals that deviate from the traditional faculty path experience "self-doubt, frustration, and resentment" (p. 21). My findings suggest that the relationship between career motivation and satisfaction for part-time $\mathrm{PhD}$ students is more closely aligned with the findings of Pifer and Baker (2014) rather than Sakurai et al. (2017), which is likely due to the difference in career motivation in my study's participants (i.e., the majority pursuing alt-ac careers).

As predicted by Pifer and Baker (2014), many part-time students in my study indicated feeling frustrated and disappointed with the career preparatory aspects of their $\mathrm{PhD}$ programs. This frustration likely stems from misconceptions about the purpose and structure of $\mathrm{PhD}$ curriculum. Students who enter PhD programs with expectations that the curriculum will prepare them for administrative work in ways similar to professional degrees (e.g., EdDs, MBAs) are likely to be disappointed. Heflinger and Doykos (2016) found that doctoral programs are not equipped to prepare students for careers outside of the traditional faculty trajectory (i.e., alt-ac careers). More importantly, such alt-ac professional preparation is not the purpose of the research doctorate. If students pursue a degree for reasons outside of the degree's own purpose, it is unsurprising that they would be dissatisfied overall with the experience.

Regardless of ultimate career goals (faculty or alt-ac), part-time participants in my study discussed their frustration with how frequently publishing and presenting were emphasized and used as markers of success. For many participants, this frustration seemed to derive from a feeling that they were overworked and not logistically capable of 
taking on additional tasks. For others, however, the frustration stemmed from a perceived misalignment between the purpose of the degree and current labor markets. That is, many participants noted the increasing decline in availability of faculty careers (Flaherty, 2020), which made them question the overall purpose of the PhD as a method of faculty preparation. Taken together, these findings indicate that an emphasis on traditional metrics of academic career preparation may be detracting from part-time $\mathrm{PhD}$ student satisfaction. The negative relationship between satisfaction and academic career preparation was common among all part-time students seeking alt-ac careers, regardless of their interest in developing academic skills (e.g., research and analytical abilities).

At the same time, the lack of socialization that affects many part-time students may also be affecting their views of publishing and presenting. Part-time students in my study tended to discuss publishing and presenting in terms of faculty career preparation without consideration of the purpose behind peer-reviewed publishing (e.g., validation, dissemination). In my study, part-time participants were primarily interested in conducting research for practical application, but many participants seemed to believe that such application did not require that the research be peer-reviewed. This represents a fundamental misunderstanding of the purpose of peer-review. Although not without its criticisms (e.g., Smith, 2006, Lee et al., 2013), peer-review is a critical process in ensuring research validity (Cowell, 2014; Kassirer et al., 1994). A lack of awareness about the purpose of peer review further supports the finding that part-time students may not be properly socialized into their disciplines.

\section{Implications for Research and Practice}

Part-time students are a growing population (Department of Education, 2016) and the scarcity of research about their needs and experiences represents a considerable gap 
in the literature about doctoral education and non-traditional student support. My findings make progress towards filling that gap and suggest several implications for research and practice.

\section{Implications for Research}

Findings from this study generate numerous implications for research, as this student population remains considerably understudied and much remains unknown about the part-time $\mathrm{PhD}$ student experience. In this section, I present key recommendations for future research centered around three areas that shape part-time $\mathrm{PhD}$ student experiences: student, faculty, and doctoral programs.

Students. Perhaps more than anything, my findings indicate a need for further research into the part-time $\mathrm{PhD}$ student population. Part-time $\mathrm{PhD}$ students are excluded from major inquiries into doctoral education in the United States (e.g., the Survey of Earned Doctorates), and the future of doctoral education should consider the effects of enrollment status on doctoral student experiences and outcomes. Such research should examine part-time student enrollment and persistence, as currently there are no national data that show how many students are enrolling in PhD programs on a part-time basis or how this enrollment affects their overall persistence. Similarly, national data about parttime $\mathrm{PhD}$ employment outcomes could aid in building knowledge around the career goals and outcomes of this student population, as my findings indicate that many part-time students may not be pursuing traditional faculty roles, and conflicts in career goals and values between students and programs may lead to undesirable outcomes (e.g., dissatisfaction, program withdrawal). Many of these data are likely already available in the Survey of Earned Doctorates, but without identifying information regarding enrollment status, it is impossible to delineate part-time student experiences from full- 
time student experiences. Therefore, instruments used in studying doctoral education should be revised to account for enrollment status.

Beyond the baseline data that enrollment and persistence rates can provide, research should also consider how aspects of the doctoral student experience differ for part-time students. For instance, the literature about doctoral education emphasizes the importance of socialization to doctoral student success, and my findings indicate that part-time PhD students may not be properly socialized into their programs and disciplines. However, as socialization was not the primary purpose of my study, more targeted research is needed in order to understand how, or if, part-time PhD students are experiencing socialization. Similarly, although examining the effects of identity on the doctoral student experience was not the purpose of this study, findings indicate that gender, race/ethnicity, and socio-economic status may affect the part-time student experience, as participants frequently spoke of how their individual and familial characteristics influenced their decision to enroll part-time and their ability to complete degree requirements. Given that the literature about doctoral education also speaks overwhelmingly of how identity affects doctoral student experiences broadly, future research should focus on how identity shapes the experiences of part-time $\mathrm{PhD}$ students.

Finally, my findings provide guidance around best practices when studying the part-time $\mathrm{PhD}$ student population. When studying career motivation, for instance, my findings indicate a need for nuance in developing career-related survey items. The MPhD survey instrument (Litalien et al., 2015) includes career items that are exclusively extrinsic (e.g., promotion, raise), yet the findings and the work of Sakurai et al. (2017) shows that career related motivations are likely both intrinsic and extrinsic, and thus future instruments should account for these differences in order to understand the 
relationship between career advancement and $\mathrm{PhD}$ motivation. In terms of data collection, my study showed that part-time students tended to have lots of information to share. For instance, one of my part-time exclusive focus groups ran nearly $150 \%$ of the allotted time ( $\sim 90$ minutes) despite only having 5 participants. Future research should account for the possibility of lengthy responses in study designs.

Faculty. In addition to research on student characteristics and experiences, there is a need for research about faculty perceptions, trainings, and experiences related to parttime PhD students. My findings indicate that many part-time students believe faculty view them as inferior to full-time students in a myriad of ways, but my study did not include faculty perspectives on this issue. Future research into faculty perspectives could help illuminate whether my findings are due to a lack of socialization or misunderstanding among the students or if faculty tend to view and treat part-time students differentially. Many of the students' perceptions of faculty roles seemed to be rooted in a belief that faculty did not understand what it was like to complete a PhD parttime, with the assumption being that faculty likely all completed their degrees full-time. While research into the career outcomes of part-time $\mathrm{PhD}$ students could help either dispel or confirm this assumption, more research is needed about faculty training to understand why students believe faculty do not understand their experiences, regardless of the faculty's individual background. Just as faculty are trained to advise and mentor full-time doctoral students, research should also examine how faculty are trained (or not) to work with part-time doctoral students. Given the limitations of faculty workloads, research should examine how faculty, particularly at research universities, are incentivized and prepared to engage with part-time students. 
Research into faculty experiences and perceptions should also examine how faculty are responding to the professionalization of the PhD. Findings from my study indicate that many part-time $\mathrm{PhD}$ students believe the purpose of the $\mathrm{PhD}$ is to advance one's career or to gain professional legitimacy. Although this belief clearly contradicts with traditional conceptualizations around the PhD's purpose (i.e., to create and disseminate new knowledge in one's field), there is currently a lack of research about how faculty are understanding and responding to shifts in student perceptions of the degree. Research into this area will likely vary considerably between disciplines. Although my findings indicate that the tendency to view the degree as a method of professional advancement occurs across disciplines, it is particularly common in the social sciences. Understanding faculty perceptions of the purpose of the degree can also help contextualize programmatic decisions that shape the degree's curriculum.

Doctoral Programs. The policies and structures of current doctoral programs are enabling students to complete PhDs through part-time enrollment (e.g., having classes at night), yet little is known about why programs admit students in this way and how this decision affects the programs themselves or the institutions that house them. For instance, very little research has been done about the costs and benefits associated with institutional policies that enable employees to pursue part-time doctoral education (e.g., tuition remission, employee flex time), yet my findings indicate that the vast majority of part-time PhD students are not only using these policies to complete these degrees, but also identifying the existence of the policies (particularly tuition remission) as one of their primary sources of motivation. Compounding this issue, many of the part-time students using tuition remission were also studying fields directly related to their professional responsibilities (e.g., Higher Education, College Student Personnel) and 
conducting research that would directly benefit the institution itself through addressing current programmatic issues. Future research should take such factors into account and examine the return on investment associated with tuition remission and associated policies.

Other research into doctoral programs should examine how discipline-specific aspects of the doctoral program influence the part-time PhD student experience. For example, students of all disciplines described feeling as if they were a part-time anomaly in a primarily full-time program, yet my findings indicate that many programs in the social sciences (education, social work, public health) have a considerable number of part-time students. Therefore, future research should examine how and why certain disciplines attract higher percentages of part-time students and what programmatic changes these enrollment patterns may require. Similarly, discipline specific research should examine how the credential of the $\mathrm{PhD}$ is being valued and used in alt-ac career paths. Findings indicate that many part-time students believe that the $\mathrm{PhD}$ is required for professional advancement, and thus discipline-specific research should examine whether this belief is founded on labor market changes and whether such changes necessitate a reassessment of program curriculum, structure, or policies. All of these issues also provide evidence for needed practice-based changes.

\section{Implications for Practice}

I identified several implications for practice in doctoral education related to the needs of part-time PhD students. While there are likely practical implications for the students (e.g., understanding the purpose of the degrees they are pursuing), my practicebased implications center on aspects of the $\mathrm{PhD}$ student experience that are external to the students themselves yet shape the student experience. Specifically, I present 
implications related to the institutions and programs that admit part-time students and the faculty that work with them.

Institutions. Part-time students in my study expressed a universal desire for equity in institutional supports. At typical research universities, doctoral studies are housed within the academic unit; however, it is also common for there to be a crossdisciplinary organizing unit (e.g., the Graduate School) whose purpose is to oversee admissions, policies, and support functions. For instance, it might be common for graduate schools to offer a handbook or student social for new students. Additionally, many units exist to support the academic unit (the library, the student health center). Both organizing and supplementary units frequently offer events and resources for doctoral students, yet part-time students in my study identified feeling excluded from participating in such events, as they are typically held during business hours. University units should consider their total student body when designing these auxiliary services in order to ensure equity across student populations. Specifically, university units should make efforts to schedule events when the majority of enrolled students are able to participate, which would likely entail hosting events on evenings and weekends. If events must be scheduled during business hours, university units should consider providing options for virtual attendance or recording the event for later viewing.

Institutions should also consider the effects and outcomes of part-time $\mathrm{PhD}$ study when developing policies that enable students to pursue PhDs part-time. For instance, tuition remission and flex leave policies should be considered in relation to the additional work required to complete a $\mathrm{PhD}$ while working full-time. Given that using these policies in order to pursue a doctorate degree while working full-time will affect either an employee's job or academic performance (e.g., if a conflict arises between work and class 
times), supervisor support may be necessary to allow part-time students to be successful in their programs. Such support may also help facilitate mutually beneficial research between students and employing units, as findings indicate students are performing research related to their job functions. Programs admitting part-time students should consider requiring that all students who work full-time while completing the degree have the written support of their work supervisors. There are similar policy implications related to the programs themselves.

Programs or Departments. Doctoral programs should consider whether admissions and graduation policies are applied equitably to full-time and part-time students. For instance, if program curriculum follows traditional models of preparing students exclusively for future faculty positions, a student's desire to pursue such a position should be factored into their admission decision, as findings indicate that dissatisfaction stems from a misalignment between one's own career goals and the career preparatory aspects of the doctoral programs. While it may not be in the best interest of the programs to revise curriculum to include alt-ac career preparation, it should be a responsibility of the admissions committee to clearly and directly explain these aspects of the program's structure to potential applicants. Similarly, if programs retain publishing and presenting at conferences as key aspects of doctoral education, then these aspects should be included in degree requirements. The current structure of expecting students to publish in journals and present at conferences without requiring them leads many parttime students to disregard these tasks and thus not experience a fundamental aspect of doctoral-level work.

Like institutions themselves, academic departments should also consider part-time student needs when developing extra-curricular events and supports and programmatic 
materials. As my findings show, some students are currently receiving materials and information that are tailored exclusively to full-time students, and this experience led students to describe feelings of frustration and alienation. Programs should ensure that all programmatic materials (e.g., programs of study) are fully applicable to both full- and part-time student needs; when appropriate, these materials should contain separate information specifically tailored to part-time enrollment. Students also expressed feeling disconnected from their peers, despite the actual percentage of part-time students in their specific programs. Extra-curricular activities should seek to further involve part-time students in department events and work to connect part-time students with each other to help alleviate feelings of isolation. Establishing virtual support networks and other virtual events may help part-time students build community despite their frequently full schedules.

Faculty. Echoing the literature about doctoral education, findings indicate that strong faculty relationships are critical to part-time $\mathrm{PhD}$ student satisfaction, and thus likely also critical to the success (e.g., graduation) of these students. Given that many of the current sources of dissatisfaction around faculty relationships stem from a lack of time and access, there needs to be further consideration about how faculty are enabled to successfully advise part-time $\mathrm{PhD}$ students. The current structure of faculty evaluation which prioritizes publishing, teaching, and service over one-on-one advising does not incentivize faculty to work with part-time students, and while many faculty may wish to spend more time with their students, the realities of increased workloads in other areas makes such time prohibitive. All units involved in evaluating faculty work (e.g., departments, colleges, institutions) should consider how current priorities negate advising for part-time students and factor in these priorities during admissions decisions. 
Beyond the primary issues associated with time and work, implications also suggest a greater need for dialog between faculty and part-time students. Findings indicate that many part-time students do not understand faculty roles and, on the other hand, believe that faculty do not understand the part-time experience. While more data is needed to confirm or refute the latter assumption, the beliefs of these students indicate that more cross-communication is needed in order for these students to understand and feel integrated into their departmental and disciplinary cultures. Addressing issues in advising and working to better include part-time students in departmental events would make progress in this area, and beyond that faculty should work together to identify whether part-time PhD students are being fully considered in their programmatic decisions and structures (e.g., course offerings, travel funding, degree requirements).

\section{Conclusion}

This study investigated the relationship between motivation and satisfaction for part-time PhD students at one research intensive university in the mid-western United States through a mixed-methods design and guided by the theoretical framework of selfdetermination theory (Deci \& Ryan, 1985). I collected quantitative data from a survey using the Motivation for PhD Studies scale (Litalien et al, 2015) and the Doctoral Student Satisfaction Scale (Dericks et al., 2019). I collected qualitative data through a series of eight semi-structured focus groups. In total, 430 students participated in the quantitative portion, and 50 students participated in the qualitative portion.

Findings largely adhered to previous literature about doctoral education and the part-time PhD student experience. Quantitative findings supported the application of selfdetermination theory to part-time $\mathrm{PhD}$ students, indicating that there was a significant positive relationship between autonomous motivation and satisfaction and a significant 
negative relationship between controlled motivation and satisfaction. Quantitative findings further found that enrollment was not a significant predictor of satisfaction, and part-time students report higher levels of satisfaction than full-time students. Qualitative findings built upon the quantitative portion through adding nuance to the relationship between motivation and satisfaction for part-time $\mathrm{PhD}$ students. The combined findings led to three assertions about the relationship between motivation and satisfaction for parttime PhD students: (a) community motivates part-time students, but access to community may be limited; (b) untested assumptions about the $\mathrm{PhD}$ and the part-time student experience detract from overall satisfaction; and (c) part-time student career motivation is both intrinsic and extrinsic.

The findings from this study indicate that further research is needed about parttime $\mathrm{PhD}$ students. As this student population continues to grow (Department of Education, 2016), further research should examine how part-time PhD students are being socialized into their programs and disciplines. The continued decline of academic jobs (Flaherty, 2020) also suggests that future research should look at professional outcomes of part-time $\mathrm{PhD}$ students and alignment between $\mathrm{PhD}$ curriculum and alt-ac positions.

With continued research, part-time $\mathrm{PhD}$ student experiences can become a normalized sub-population within the literature about and practices of doctoral education. Institutions, programs, and faculty can work together to support part-time $\mathrm{PhD}$ students in their doctoral pursuits, even if this pursuit differs from traditional models of doctoral education. Such support will increase access to doctoral programs and allow students who might not otherwise be able to pursue the $\mathrm{PhD}$ to reach the highest levels of education. Perhaps more importantly, considering part-time $\mathrm{PhD}$ student needs and experiences can help these students feel valued and accepted in their scholarly 
communities. In this way, part-time $\mathrm{PhD}$ students can begin to feel that they, too, are valid. 


\section{REFERENCES}

Bair, C. R., \& Haworth, J. G. (1999). Doctoral Student Attrition and Persistence: A MetaSynthesis of Research. ASHE Annual Meeting Paper.

Baker, V. L., \& Lattuca, L. R. (2010). Developmental networks and learning: Toward an interdisciplinary perspective on identity development during doctoral study. Studies in Higher Education, 35(7), 807-827.

Baker, V. L., \& Pifer, M. J. (2011). The role of relationships in the transition from doctoral student to independent scholar. Studies in Continuing Education, 33(1), 5-17.

Baker, V. L., \& Pifer, M. J. (2015). Antecedents and outcomes: Theories of fit and the study of doctoral education. Studies in Higher Education, 40(2), 296-310.

Bandura, A. (1996). Self-efficacy: The exercise of control. Freeman.

Bargar, R. R., \& Mayo-Chamberlain, J. (1983). Advisor and advisee issues in doctoral education. The Journal of Higher Education, 54(4), 407-432.

Barnes, B. J., \& Randall, J. (2012). Doctoral student satisfaction: An examination of disciplinary, enrollment, and institutional differences. Research in Higher Education, 53(1), 47-75.

Barr, A., \& Turner, S. E. (2013). Expanding enrollments and contracting state budgets: The effect of the Great Recession on higher education. The ANNALS of the American Academy of Political and Social Science, 650(1), 168-193.

Bates, P., \& Goff, L. (2012). The invisible student: Benefits and challenges of part-time doctoral studies. Alberta Journal of Educational Research, 58(3), 368-380.

Bell, M. P. (2009). Effects of the experience of inequality, exclusion and discrimination on scholarship. Equality, Diversity and Inclusion at Work: A Research 
Companion, 17-26.

Berry, S. (2017). Student support networks in online doctoral programs: Exploring nested communities. International Journal of Doctoral Studies, 12, 33.

Biglan, A. (1973a). The characteristics of subject matter in different academic areas. Journal of Applied Psychology 57(3).

Bragg, A. K. (1976). The socialization process in higher education. George Washington University.

Burd, S. (1996, September 6). Murder of Three Professors at a Thesis Defense Stuns Campus. The Chronicle of Higher Education. https://www.chronicle.com/article/murder-of-3-professors-at-a-thesis-defensestuns-campus/

Chen, P. S. D., Lambert, A. D., \& Guidry, K. R. (2010). Engaging online learners: The impact of Web-based learning technology on college student engagement. Computers \& Education, 54(4), 1222-1232.

Choy, S. P., \& Cataldi, E. F. (2006). Student financing of graduate and first-professional education, 2003- 04. National Center for Education Statistics (NCES 2006-185).

Cohen, B. H. (2008). Explaining psychological statistics. John Wiley \& Sons.

Collins, C. S., \& Stockton, C. M. (2018). The central role of theory in qualitative research. International Journal of Qualitative Methods, 17(1), 1609406918797475.

Coolidge, H.J. (1932). Archibald Cary Coolidge: Life and Letters. Books for libraries. Council of Graduate Schools. (2007). Ph.D. completion and attrition: Policy, numbers, leadership, and next steps. Washington, DC: Author.

Council of Graduate Schools (2012). Global perspectives on career outcomes for 
graduate students: Tracking and building pathways. Missing?

Creswell, J. W. (2002). Educational research: Planning, conducting, and evaluating quantitative research. Prentice Hall.

Creswell, J. (2009). Research design: Qualitative, quantitative, and mixed methods approaches. Sage.

Creswell, J. (2012). Educational research: Planning, conducting, and evaluating quantitative and qualitative research. Pearson.

Creswell, J., \& Plano Clark, V. (2011). Designing and conducting mixed methods research. Sage.

Crumb, L., Haskins, N., Dean, L., \& Avent Harris, J. (2019). Illuminating social-class identity: The persistence of working-class African American women doctoral students. Journal of Diversity in Higher Education.

Curran, C. C. (1987). Dealing with the distant learner as part-time learner. Journal of Education for Library and Information Science, 27, 240-246.

Deem, R., \& Brehony, K. J. (2000). Doctoral students' access to research cultures - Are some more unequal than others? Studies in Higher Education, 25, 149-165.

Deci, E. L., \& Ryan, R. M. (1985). Intrinsic motivation and self-determination in human behavior. Plenum Press.

Deci, E. L., \& Ryan, R. M. (2012). Motivation, personality, and development within embedded

social contexts: An overview of self-determination theory. In R. M. Ryan (Ed.), The Oxford Handbook of Human Motivation, 85-107. Oxford University Press.

Deering, T. E. (1998). Eliminating the doctor of education degree: It's the right thing to do. The Educational Forum, 62, 3, 243-248. 
Dey, I. (1993). Qualitative Data Analysis: A User-Friendly Guide for Social Scientists. Routledge.

Dericks, G., Thompson, E., Roberts, M., \& Phua, F. (2019). Determinants of PhD student satisfaction: the roles of supervisor, department, and peer qualities. Assessment $\&$ Evaluation in Higher Education, 44(7), 1053-1068.

Dickey, C. (2019). The academic job market is a nightmare. Here's one way to fix it. The Washington Post. https://www.washingtonpost.com/outlook/2019/04/15/jobmarket-academics-is-nightmare-heres-one-way-fix-it/

Diener, E., Suh, E. M., Lucas, R. E., \& Smith, H. L. (1999). Subjective well-being: Three decades of progress. Psychological Bulletin, 125, 276-302.

Dillman, D. A., Smyth, J. D., \& Christian, L. M. (2014). Internet, phone, mail, and mixed-mode surveys: the tailored design method. John Wiley \& Sons.

Docere (n.d.). The Latin Dictionary. http://latindictionary.wikidot.com/verb:docere

Donohue, T. L., \& E. H. Wong, E. H. (1997). Achievement motivation and college satisfaction in traditional and nontraditional students. Education 118(2), 237-244.

Dressel, P. L., \& Mayhew, L. B. (1974). Higher education as a field of study: The emergence of a profession. Jossey-Bass.

Ellis, E. M. (2001). The impact of race and gender on graduate school socialization, satisfaction with doctoral study, and commitment to degree completion. Western Journal of Black Studies, 25(1), 30.

Espino, M. M., Muñoz, S. M., \& Marquez Kiyama, J. (2010). Transitioning from Doctoral Study to the Academy: Theorizing Trenzas of Identity for Latina Sister Scholars. Qualitative Inquiry, 16(10), 804-818.

Felder, P. (2010). On doctoral student development: Exploring faculty mentoring in the 
shaping of African American doctoral student success. Qualitative Report, 15(2), $455-474$.

Felder, P. P., Stevenson, H. C., \& Gasman, M. (2014). Understanding race in doctoral student socialization. International Journal of Doctoral Studies, 9(19), 21-42.

Flaherty, C. (2020). Calm before the storm. Inside Higher Ed.

https://www.insidehighered.com/news/2020/12/09/nsf-data-doctorates-grantedshow-slow-growth-some-exceptions

Forbus, P., Newbold, J. J., \& Mehta, S. S. (2011). A study of non-traditional and traditional students in terms of their time management behaviors, stress factors, and coping strategies. Academy of Educational Leadership Journal, 15, 109.

Freeman Jr, S., Hagedorn, L. S., Goodchild, L., \& Wright, D. (2013). Advancing higher education as a field of study: In quest of doctoral degree guidelines -commemorating 120 years of excellence. Stylus Publishing.

Gaff, J. G., \& Pruitt-Logan, A. S. (1998). Preparing college faculty. New Directions for Higher Education, 101, 77-86.

Gardner, S. K. (2008). Fitting the mold of graduate school: A qualitative study of socialization in doctoral education. Innovative Higher Education, 33, 125-138.

Gardner, S. K. (2009). Conceptualizing success in doctoral education: Perspectives of faculty in seven disciplines. The Review of Higher Education, 32(3), 383-406.

Gardner, S. K., \& Gopaul, B. (2012). The part-time doctoral student experience. International Journal of Doctoral Studies, 7(12), 63-78.

Gardner, S. K., \& Holley, K. A. (2011). "Those invisible barriers are real”: The experiences of first-generation doctoral students. Equity and Excellence in Education, 44, 77-92. 
Gerholm, T. (1990). On Tacit Knowledge in Academia. European Journal of Education, 25(3), 263- 271.

Gibson, G. W. (1992). Good start: A guidebook for new faculty in liberal arts colleges. Anker.

Golde, C. M. (1996). How Departmental Contextual Factors Shape Doctoral Student Attrition. [Doctoral Dissertation, Stanford University]. ProQuest Dissertations Publishing.

Golde, C. M., \& Dore, T. M. (2001). At cross purposes: What the experiences of today's doctoral students reveal about doctoral education. Pew Charitable Trusts.

Golde, C. M. (2005). The role of the department and discipline in doctoral student attrition: Lessons from four departments. The Journal of Higher Education, 76(6), $669-700$.

Greene, J. C., Caracelli, V. J., \& Graham, W. F. (1989). Toward a conceptual framework for mixed-method evaluation designs. Educational evaluation and policy analysis, 11(3), 255-274.

Greene, M. (2015). Come hell or high water: Doctoral students' perceptions on support services and persistence. International Journal of Doctoral Studies, 10, 501-518.

Guba, E. (1990). The paradigm dialog. Sage.

Hall, S. S. (1998, November 29). Lethal Chemistry at Harvard. The New York Times Magazine. https://www.nytimes.com/1998/11/29/magazine/lethal-chemistry-atharvard.html

Hartnett, R. T. (1976). Environment for Advanced Learning. In J. Katz \& R. T. Hartnett (Eds.), Scholars in the Making: The Development of Graduate and Professional Students, 49-84. Ballinger Publishing. 
Heflinger, C. A., \& Doykos, B. (2016). Paving the pathway: Exploring student perceptions of professional development preparation in doctoral education. Innovative higher education, 41(4), 343-358.

Holahan, C. K. (1979). Stress experienced by women doctoral students, need for support, and occupational sex typing: An interactional view. Sex Roles, 5, 425-436.

Holley, K. A., \& Gardner, S. K. (2012). Navigating the pipeline: How socio-cultural influences impact first-generation doctoral students. Journal of Diversity in Higher Education, 5, 112-121.

Holmes, B., Brown, L., Parker, D., Mann, J., Woods, E., Gibson, J. A., Hall, D. (2016). Decoding the Persistence and Engagement Patterns of Doctoral Students Who Finish. Hampton University School of Education and Human Development.

Hull, C.L. (1943). Principles of behavior: An introduction to behavior theory. AppletonCentury-Crofts

Hyle, A. \& Goodchild, L. (2014). Contemporary conditions of higher education programs in the United States and Canada. In L. Wolf-Wendel (Ed.) Advancing higher education as a field of study: In quest of doctoral degree guidelinesCommemorating 120 Years of excellence. Stylus Publishing.

Ivankova, N., \& Stick, S. (2007). Students' persistence in a distributed doctoral program in educational leadership in higher education: A mixed methods study. Research in Higher Education, 48, 93-135.

Ives, G., \& G. Rowley. 2005. Supervisor selection or allocation and continuity of supervision: Ph.D. students' progress and outcomes." Studies in Higher Education, 30(5), 535-555.

Jager, J., Putnick, D. L., \& Bornstein, M. H. (2017). More than just convenient: The 
scientific merits of homogeneous convenience samples. Monographs of the Society for Research in Child Development, 82(2), 13-30.

Jepsen, D. M., Varhegyi, M. M., \& Edwards, D. (2012). Academics' attitudes towards PhD students' teaching: Preparing research higher degree students for an academic career. Journal of Higher Education Policy and Management, 34(6), 629-645.

Jones, M. (2013, July). Issues in Doctoral Studies-Forty Years of Journal Discussion: Where have we been and where are we going? In Proceedings of the Informing Science and Information Technology Education Conference, 83-104. Informing Science Institute.

Karl, K., \& Peluchette, J. (2013). Management faculty perceptions of candidates with online doctorates: Why the stigma?. American Journal of Distance Education, 27(2), 89-99.

Kelly, K., Linder, K. E., \& Tobin, T. J. (2020). Going Alt-ac: A Guide to Alternative Academic Careers. Stylus Publishing, LLC.

Krueger, R. A. (2014). Focus groups: A practical guide for applied research. Sage publications.

Kvale, S. (1996). InterViews: an introduction to qualitive research interviewing. Sage.

Langford, J., \& Clance, P. R. (1993). The imposter phenomenon: recent research findings regarding dynamics, personality and family patterns and their implications for treatment. Psychotherapy: theory, research, practice, training, 30(3), 495.

Lei, S. A., \& Chuang, N.-K. (2009). Research collaboration and publication during graduate studies: Evaluating benefits and costs from students' perspectives. College Student Journal, 43(4), 1163-1168. 
Litalien, D., Guay, F., \& Morin, A. J. (2015). Motivation for PhD studies: Scale development and validation. Learning and Individual Differences, 41, 1-13.

Lovitts, B. E. (2001). Leaving the ivory tower: The causes and consequences of departure from doctoral study. Rowman \& Littlefield.

Maher, M. A., Ford, M. E., \& Thompson, C. M. (2004). Degree progress of women doctoral students: Factors that constrain, facilitate, and differentiate. The Review of Higher Education, 27(3), 385-408.

Mason, M. M. (2012). Motivation, satisfaction, and innate psychological needs. International Journal of Doctoral Studies, 7(1), 259-277.

McDonald, R. P. (1970). Theoretical foundations of principal factor analysis and alpha factor analysis. British Journal of Mathematical and Statistical Psychology, 23, $1-21$

Mendoza, P., \& Gardner, S. K. (2010). The PhD in the United States. In S. K. Gardner \& P. Mendoza (Eds.), On becoming a scholar: Socialization and development in doctoral education, 11-26. Stylus.

Miller, E. \& Seldin, P. (2014). Changing practices in faculty evaluation. American Association of University Professors. https://www.aaup.org/article/changingpractices-faculty-evaluation

Morse, J. M., \& Niehaus, L. (2009). Principles and procedures of mixed methods design. Left.

Morton, M., \& G. Thornley. (2001). Experiences of doctoral students in mathematics in New Zealand. Assessment \& Evaluation in Higher Education 26 (2), 113-126.

Mu, K., Coppard, B. M., Bracciano, A. G., \& Bradberry, J. C. (2014). Comparison of oncampus and hybrid student outcomes in occupational therapy doctoral education. 
American Journal of Occupational Therapy, 68, S51-S56.

Murakami-Ramalho, E., Militello, M., \& Piert, J. (2013). A view from within: How doctoral students in educational administration develop research knowledge and identity. Studies in Higher Education, 38(2), 256-271.

Nettles, M. T. (1990). Black, Hispanic, and White Doctoral Students: Before, During, and After Enrolling in Graduate School. Educational Testing Service.

Nettles, M. T., \& Millett, C. M. (2006). Three magic letters: Getting to Ph.D. Johns Hopkins University Press.

Neumann, R., \& Rodwell, J. (2009). The 'invisible' part-time research students: A case study of satisfaction and completion. Studies in Higher Education, 34, 55-68.

Nimer, M. (2009). The doctoral cohort model: Increasing opportunities for success. College Student Journal, 43, 1373-1379.

Nora, A., \& Snyder, B. P. (2007). Structural differences in scholarly engagement among full- and part-time doctoral students. Paper presented at the Annual Meeting of the American Educational Research Association, Chicago, IL.

O'Connor, B. N., \& Cordova, R. (2010). Learning: The experiences of adults who work full-time while attending graduate school part-time. Journal of Education for Business, 85(6), 359-368.

Offerman, M. (2011). Profile of the nontraditional doctoral degree student. New Directions for Adult and Continuing Education, (129), 21-30.

Osborne, J. W. (2014). Best practices in logistic regression. Sage Publications. Osborne, J. W. (2016). Regression \& linear modeling: Best practices and modern methods. Sage.

O'Toole, D. M., Stratton, L. S., \& Wetzel, J. N. (2003). A longitudinal analysis of the 
frequency of part-time enrollment and the persistence of students who enroll part time. Research in Higher Education, 44(5), 519-537.

Ott, M. D., \& Markewich, T. S. (1985). Logit Analysis of Graduate Student Retention and Graduation. Paper presented at the Annual Forum of the Association for Institutional Research, Portland, OR.

Padmalochanan, P. (2019). Academics and the field of academic publishing: challenges and approaches. Publishing Research Quarterly, 35(1), 87-107.

Pauley, R., Cunningham, M., \& Toth, P. (1999). Doctoral student attrition and retention: A study of a non-traditional EdD. program. Journal of College Student Retention, $1,225-238$.

Pike, G. R. 1993. The Relationship between Perceived Learning and Satisfaction with College: An Alternative View. Research in Higher Education 34(1), 23-40.

Pilbeam, C., and D. Denyer. 2009. Lone Scholar or Community Member? The Role of Student Networks in Doctoral Education in a UK Management School. Studies in Higher Education 34(3), 301-318.

Pintrich, P. R. (2003). A motivational science perspective on the role of student motivation in learning and teaching contexts. Journal of Educational Psychology, $95,667-686$.

Pyhältö, K., Toom, A., Stubb, J., \& Lonka, K. (2012). Challenges of becoming a scholar: A study of doctoral students' problems and well-being. International Scholarly Research Network Education, 12, 1- 12.

Pifer, M. J., \& Baker, V. L. (2016). Stage-based challenges and strategies for support in doctoral education: A practical guide for students, faculty members, and program administrators. International Journal of Doctoral Studies, 11(1), 15-34. 
Ravitch, S. M., \& Carl, N. M. (2016). Validity: Process, strategies, and considerations. Qualitative research: Bridging the conceptual, theoretical, and methodological, 185-214.

Rawat, S., \& Meena, S. (2014). Publish or perish: Where are we heading?. Journal of research in medical sciences: the official journal of Isfahan University of Medical Sciences, $19(2), 87$.

Riedling, A. M. (1997). An exploratory study: Distance education doctoral students in the field of educational policy studies and evaluation at the University of Kentucky. [Doctoral Dissertation, University of Louisville]. ProQuest Dissertations Publishing.

Rigler, K. L., Bowlin, L. K., Sweat, K., Watts, S., \& Throne, R. (2017). Agency, socialization, and support: A critical review of doctoral student attrition. Paper presented at the 3rd International Conference on Doctoral Education, University of Central Florida.

Roberts, J., \& Styron, R., Jr. 2010. Student satisfaction and persistence: Factors vital to student retention. Research in Higher Education Journal, 1-18.

Ryan, R. M., \& Deci, E. L. (2000). Self-determination theory and the facilitation of intrinsic motivation, social development, and well-being. American Psychologist, $55,68-78$.

Saldaña, J. (2016). The coding manual for qualitative researchers. Sage.

Sakurai, Y., Vekkaila, J., \& Pyhältö, K. (2017). More or less engaged in doctoral studies? Domestic and international students' satisfaction and motivation for doctoral studies in Finland. Research in Comparative and International Education, 12(2), 143-159. 
Smith, P. R. (2000). A meeting of cultures: Part-time students in an EdD. program. International Journal of Leadership in Education, 3, 359-380.

Solmon, L. C. (1973). Women in doctoral education: Clues and puzzles regarding institutional discrimination. Research in Higher Education, 1(4), 299-332.

Solórzano, D. G. (1998). Critical race theory, race and gender microaggressions, and the experience of Chicana and Chicano scholars. International journal of qualitative studies in education, 11(1), 121-136.

Sowell, R., Allum, J., \& Okahana, H. (2015). Doctoral initiative on minority attrition and completion. Washington, DC: Council of Graduate Schools.

Sijtsma, K. (2009). On the use, misuse, and the very limited usefulness of Cronbach's alpha. Psychometrika, 74, 107-120.

Streiner, D. L. (2003). Starting at the beginning: An introduction to coefficient alpha and internal consistency. Journal of Personality Assessment, 80, 99-103.

Styron Jr, R. (2010). Student satisfaction and persistence: Factors vital to student retention. Research in Higher Education Journal, 6, 1.

Sweitzer, V. (2009). Towards a theory of doctoral student professional identity development: A developmental networks approach. The Journal of Higher Education, 80(1), 1-33.

Tashakkori, A., \& Teddlie, C. (2003). Handbook of mixed methods in social and behavioral research. Sage.

Tashakkori, A., \& Teddlie, C. (1998). Mixed methodology: Combining qualitative and quantitative approaches. Sage.

Teeuwsen, P., Ratković, S., \& Tilley, S. A. (2014). Becoming academics: Experiencing legitimate peripheral participation in part-time doctoral studies. Studies in Higher 
Education, 39(4), 680-694

Templeton, R. (2016). Doctorate motivation: An (auto)ethnography. Australian Universities Review, 39-44.

Thompson, J., Ballenger, J. N., \& Templeton, N. R. (2018). Examining Quality Elements in a High Education Fully Online Doctoral Program: Doctoral Students' Perceptions. International Journal of Educational Leadership Preparation, 13(1), 51-63.

Thune, T. (2010). The training of "triple helix workers"? Doctoral students in universityindustry-government collaborations. Minerva, 48(4), 463-483.

Townsend, B. K. (2002). Rethinking the EdD., or what's in a name? Presentation for the Study of Higher Education, Sacramento, CA

Turner, C. S. V., \& Thompson, J. R. (1993). Socializing women doctoral students: Minority and majority experiences. The review of higher education, 16(3), 355370.

Turner, J. (2018). Delivery, facilitas, and copia: Job market preparation and the revival of the fifth canon. Pedagogy, 18(3), 511-530.

Umbach, P. D., \& Porter, S. R. (2002). How do academic departments impact student satisfaction? Understanding the contextual effects of departments. Research in Higher Education 43(2), 209-234.

van Manen, M. (1997). Researching lived experience: Human science for an action sensitive pedagogy (2nd Ed.). The Althouse Press.

Vansteenkiste, M., Neyrinck, B., Niemiec, C. P., Soenens, B., De Witte, H., \& Van den Broeck, A. (2007). On the relations among work value orientations, psychological need satisfaction and job outcomes: A self-determination theory 
approach. Journal of occupational and organizational psychology, 80(2), 251277.

Watts, J. H. (2008). Challenges of supervising part-time PhD students: Towards studentcentered practice. Teaching in Higher Education, 13, 369-373.

Weidman, J. C., Twale, D. J., \& Stein, E. L. (2001). Socialization of graduate and professional students in higher education: A perilous passage? Jossey-Bass.

Weiss, C. S. (1981). The Development of Professional Role Commitment Among Graduate Students. Human Relations, 34(1), 13-31.

Wichmann-Hansen, G., \& Herrmann, K. J. (2017). Does external funding push doctoral supervisors to be more directive? A large-scale Danish study. Higher education, 74(2), 357-376.

Wisker, G., Robinson, G. D., \& Leibowitz, B. (2019). The purpose and impact of postgraduate knowledge. Studies in Graduate and Postdoctoral Education.

Wulff, D. H., Austin, A. E., Nyquist, J. D., \& Sprague, J. (2004). The development of graduate students as teaching scholars: A four-year longitudinal study. In D. H. Wulff \& A. E. Austin (Eds.), Paths to the professoriate: Strategies for enriching the preparation of future faculty, 46-73. Jossey-Bass.

Wyatt, L. G. (2011). Nontraditional student engagement: Increasing adult student success and retention. The Journal of Continuing Higher Education, 59(1), 10-20.

Zahl, S. B. (2015). The impact of community for part-time doctoral students: How relationships in the academic department affect student persistence. International Journal of Doctoral Studies, 10, 301-321.

Zhang, T., Solmon, M. A., Kosma, M., Carson, R. L., \& Gu, X. (2011). Need support, need satisfaction, intrinsic motivation, and physical activity participation among 
middle school students. Journal of teaching in physical education, 30(1), 51-68.

Zhao, C., Golde, C. M., \& McCormick, A. C. (2007). More than a signature: How advisor choice and advisor behaviour affect doctoral student satisfaction. Journal of Further and Higher Education 31(3), 263-281. 


\section{Appendix A}

\section{Alignment Between Proposed Study and Mixed Methods Recommendations}

\begin{tabular}{|c|c|c|}
\hline \multicolumn{2}{|c|}{ Purpose and Definition } & \multirow{2}{*}{$\begin{array}{l}\text { Relation to Proposed Study } \\
\text { In the qualitative portion, I } \\
\text { will seek to corroborate the } \\
\text { quantitative findings with the } \\
\text { students' personal views of } \\
\text { their motivation and } \\
\text { satisfaction }\end{array}$} \\
\hline Triangulation & $\begin{array}{l}\text { Seeks convergence, corroboration, } \\
\text { and correspondence of results from } \\
\text { the different methods }\end{array}$ & \\
\hline Complementarity & $\begin{array}{l}\text { Seeks elaboration, enhancement, } \\
\text { illustration, and clarification of the } \\
\text { results from one method with the } \\
\text { results from the other method. }\end{array}$ & $\begin{array}{l}\text { In the qualitative portion, } \\
\text { students will be asked to } \\
\text { expand on their reporting of } \\
\text { their motivation and } \\
\text { satisfaction during the } \\
\text { quantitative section in order to } \\
\text { provide clarity and context }\end{array}$ \\
\hline Development & $\begin{array}{l}\text { Seeks to use the results from one } \\
\text { method to help develop or inform } \\
\text { the other method, where } \\
\text { development is broadly construed } \\
\text { to include sampling and } \\
\text { implementation, as well as } \\
\text { measurement decisions. }\end{array}$ & $\begin{array}{l}\text { Results from the quantitative } \\
\text { portion will be used to inform } \\
\text { the development of the } \\
\text { protocol for the focus groups }\end{array}$ \\
\hline Initiation & $\begin{array}{l}\text { Seeks the discovery of paradox } \\
\text { and contradiction, new } \\
\text { perspectives of frameworks, the } \\
\text { recasting of questions or results } \\
\text { from one method with questions or } \\
\text { results from the other method. }\end{array}$ & $\begin{array}{l}\text { Due to the lack of research on } \\
\text { this topic, findings may } \\
\text { present unexpected results that } \\
\text { will be clarified and explained } \\
\text { during the qualitative phase of } \\
\text { data collection }\end{array}$ \\
\hline Expansion & $\begin{array}{l}\text { Seeks to extend the breadth } \\
\text { and range of inquiry by using } \\
\text { different methods for different } \\
\text { inquiry components. }\end{array}$ & $\begin{array}{l}\text { Motivation and satisfaction } \\
\text { have typically been studied } \\
\text { either qualitatively or } \\
\text { quantitatively and thus mixing } \\
\text { methods will provide a new } \\
\text { perspective on these constructs } \\
\text { within doctoral education }\end{array}$ \\
\hline
\end{tabular}




\section{Appendix B}

\section{Alignment Between Study Purpose, Research Questions,}

Theoretical Framework, and Instruments

\begin{tabular}{|c|c|c|c|c|}
\hline Study Purpose & $\begin{array}{l}\text { Research } \\
\text { Question }\end{array}$ & $\begin{array}{l}\text { Theoretical } \\
\text { Framework }\end{array}$ & Instruments & $\begin{array}{l}\text { Data } \\
\text { Analysis }\end{array}$ \\
\hline \multirow{3}{*}{$\begin{array}{l}\text { The purposes } \\
\text { of this study } \\
\text { were to } \\
\text { investigate the } \\
\text { relationship } \\
\text { between } \\
\text { motivation and } \\
\text { satisfaction } \\
\text { among part- } \\
\text { time PhD } \\
\text { students and to } \\
\text { build } \\
\text { knowledge } \\
\text { about the part- } \\
\text { time PhD } \\
\text { student } \\
\text { experience. }\end{array}$} & $\begin{array}{l}\text { Does motivation } \\
\text { for doctoral } \\
\text { studies influence } \\
\text { overall doctoral } \\
\text { program } \\
\text { satisfaction for } \\
\text { part-time PhD } \\
\text { students? }\end{array}$ & $\begin{array}{l}\text { Satisfaction is } \\
\text { positively } \\
\text { correlated with } \\
\text { autonomous } \\
\text { motivation and } \\
\text { negatively } \\
\text { correlated with } \\
\text { controlled } \\
\text { motivation } \\
\text { (Deci \& Ryan, } \\
\text { 1985, 2012). }\end{array}$ & $\begin{array}{l}\text { Motivation for } \\
\text { PhD Studies } \\
\text { scale (Litalien } \\
\text { et al., 2015) } \\
\text { Doctoral } \\
\text { Student } \\
\text { Satisfaction } \\
\text { scale (Dericks } \\
\text { et al., 2019) }\end{array}$ & $\begin{array}{l}\text { Hierarchical } \\
\text { multiple } \\
\text { regression }\end{array}$ \\
\hline & $\begin{array}{l}\text { How do part- } \\
\text { time PhD } \\
\text { students } \\
\text { describe their } \\
\text { motivations for } \\
\text { persisting and } \\
\text { pursuing in their } \\
\text { doctoral } \\
\text { programs? }\end{array}$ & $\begin{array}{l}\text { Motivation } \\
\text { exists on a } \\
\text { continuum } \\
\text { between } \\
\text { extrinsic and } \\
\text { intrinsic, with } \\
\text { intrinsic being } \\
\text { self-determined } \\
\text { and extrinsic } \\
\text { being } \\
\text { controlled } \\
\text { (Deci \& Ryan, } \\
\text { 1985, 2012). }\end{array}$ & $\begin{array}{l}\text { Qualitative } \\
\text { protocol } \\
\text { (Appendix D) }\end{array}$ & $\begin{array}{l}\text { Holistic and } \\
\text { eclectic } \\
\text { coding }\end{array}$ \\
\hline & $\begin{array}{l}\text { How do part- } \\
\text { time PhD } \\
\text { students } \\
\text { describe their } \\
\text { overall } \\
\text { satisfaction with } \\
\text { their doctoral } \\
\text { programs? }\end{array}$ & $\begin{array}{l}\text { Satisfaction is } \\
\text { associated with } \\
\text { other positive } \\
\text { outcomes, such } \\
\text { as well-being } \\
\text { and persistence } \\
\text { (Deci \& Ryan, } \\
\text { 2012). }\end{array}$ & $\begin{array}{l}\text { Qualitative } \\
\text { protocol } \\
\text { (Appendix D) }\end{array}$ & $\begin{array}{l}\text { Holistic and } \\
\text { eclectic } \\
\text { coding }\end{array}$ \\
\hline
\end{tabular}




\section{Appendix C}

\section{Quantitative Instrument}

Please answer the following questions to the best of your ability. Your answers will be kept confidential. Any questions should be directed to heather.turner@louisville.edu.

1. What is your age?
a. $<25$
b. $25-34$
c. $35-44$
d. $45-54$
e. $55-64$
f. $65+$

2. What is your gender?
a. Male
b. Female
c. Trans-male
d. Trans-female
e. Non-binary
f. Prefer not to answer
g. Other:

3. What is your race/ethnicity?
a. White/Caucasian
b. Asian
c. Hispanic/Latino
d. Black/African American
e. Hawaiian/Pacific Islander
f. Native American
g. Other:

4. As of today, what is your marital status?
a. Single
b. Married/Partnered
c. Divorced/Separated
d. Widowed
e. Other:

5. As of today, do you have dependents that you care for at least $50 \%$ of the time?
a. Yes
b. No

6. What is the start date of your doctoral program?

a. Drop-down with semesters (fall, winter, spring, summer) and years (2000-2020)

7. Please indicate your enrollment status in your doctoral program

a. Completely part-time ( $<9$ credit hours every semester of coursework)

b. Mostly part-time ( $<9$ credit hours most semesters of coursework)

c. Mostly full-time (9+ credit hours most semesters of coursework)

d. Completely full-time (9+ credit hours every semester of coursework) 
8. Please indicate your current stage in your doctoral program
a. Coursework
b. Comprehensive exams
c. Dissertation
d. Other:

9. Which doctoral program are you enrolled in?

a. Anatomical Science and Neurobiology

b. Biochemistry and Molecular Genetics

c. Biology

d. Biostatistics

e. Chemical Engineering

f. Chemistry

g. Civil Engineering

h. Clinical Psychology

i. Computer Science and Engineering

j. Counseling and Personnel Services

k. Criminal Justice

1. Curriculum and Instruction

m. Educational Leadership and Organizational Development

n. Electrical Engineering

o. English Rhetoric and Composition

p. Entrepreneurship

q. Experimental Psychology

r. Humanities

s. Industrial Engineering

t. Interdisciplinary Studies

u. Interdisciplinary Studies: Specialization in Bioinformatics

v. Interdisciplinary Studies: Specialization in Translational Bioengineering

w. Interdisciplinary Studies: Specialization in Translational Neuroscience

x. Mathematics, Applied and Industrial

y. Mechanical Engineering

z. Microbiology and Immunology

aa. Nursing

bb. Pan-African Studies

cc. Pharmacology and Toxicology

dd. Physics

ee. Physiology

ff. Public Health Sciences: Specialization in Environmental Health

gg. Public Health Sciences: Specialization in Epidemiology

hh. Public Health Sciences: Specialization in Health Management and Policy

ii. Public Health Sciences: Specialization in Health Promotion and Behavioral Sciences

jj. Social Work

kk. Sociology, Applied

11. Urban and Public Affairs

10. As of today, what is your employment status?

a. Employed part-time (one job only) 

b. Employed part-time (multiple jobs)
c. Employed full-time (one job)
d. Employed full-time (multiple jobs)
e. Unemployed
f. Other:

11. As of today, who is your primary employer?
a. An employer other than the university (includes self-employed)
b. The university (not an assistantship)
c. The university (in an assistantship)
d. Unemployed/Not applicable
e. Other:

12. Upon graduation, do you plan to pursue a full-time faculty position?
a. Yes
b. No
c. Undecided

13. As of today, how are you funding your doctoral program?
a. Graduate assistantship
b. Graduate fellowship
c. University tuition-remission
d. Non-university employer assistance
e. Student loans
f. Scholarships/Grants
g. Self-funded
h. Other:

The following 15 statements are from the Motivation for PhD studies scale (Litalien et al, 2015) and correspond to reasons that can motivate doctoral students to persevere in their studies. Please indicate the extent to which each statement corresponds to the reasons why you persevere in your doctoral studies according to the following scale:

\begin{tabular}{|l|l|l|l|l|}
\hline $\begin{array}{l}\text { Does not } \\
\text { correspond at all } \\
(1)\end{array}$ & $\begin{array}{l}\text { Corresponds } \\
\text { somewhat (2) }\end{array}$ & $\begin{array}{l}\text { Corresponds } \\
\text { moderately well (3) }\end{array}$ & $\begin{array}{l}\text { Corresponds } \\
\text { well (4) }\end{array}$ & $\begin{array}{l}\text { Corresponds } \\
\text { exactly (5) }\end{array}$ \\
\hline
\end{tabular}

Intrinsic

1. For the satisfaction I feel when I surpass myself in my learning activities (e.g., work, presentations).

2. For the satisfaction I have in facing challenges in my studies.

3. For the pleasure I feel in accomplishing my study project (e.g., thesis). Integrated

1. Because doctoral studies are consistent with my values (e.g., curiosity, ambition, success).

2. Because my doctoral studies are a fundamental part of who I am and my identity.

3. Because my doctoral studies meet my goals and my objectives in life. 
Identified

1. Because I want to improve my skills in my field of study.

2. Because it's important for me to advance knowledge in my field of study.

3. Because I have the opportunity to take my first steps in research (e.g., Introjected publications, collaborations) while benefitting from supervision.

1. Because my supervisor would be disappointed or angry if I gave up.

2. Because I have made commitments that I must fulfill (e.g., with funding agencies, employers, collaborators, a research director).

3. Because I do not want to be perceived as a quitter.

\section{External}

1. For the prestige associated with a PhD.

2. To find a job with good working conditions.

3. To get a better paying job after graduation.

The final set of questions come from the Doctoral Student Satisfaction scale (Dericks et al., 2019). When answering these questions, please think about your overall experience of your $\mathrm{PhD}$ to date. Please rate your level of agreement with the following words on this six-point Likert scale:

\begin{tabular}{|c|c|c|c|c|c|}
\hline $\begin{array}{c}\text { Strongly } \\
\text { Disagree } \\
(1)\end{array}$ & $\begin{array}{c}\text { Disagree } \\
(2)\end{array}$ & $\begin{array}{c}\text { Somewhat } \\
\text { Disagree }\end{array}$ & $\begin{array}{c}\text { Somewhat } \\
\text { Agree }\end{array}$ & $\begin{array}{c}\text { Agree } \\
(5)\end{array}$ & $\begin{array}{c}\text { Strongly } \\
\text { Agree (6) }\end{array}$ \\
\hline
\end{tabular}

1. Good

2. Unhappy

3. Enjoyable

4. Satisfactory

5. Bad

6. Terrible

7. Excellent

8. Disappointing

9. Happy

10. Unsatisfactory 


\section{Appendix D \\ Focus Group Interview Protocol}

Thank you for taking the time to talk with me. The purpose of this study is to understand your motivation for pursuing a PhD and your overall level of satisfaction with your doctoral program. We will begin by reviewing the informed consent, and after that I will ask you a series of questions related to your doctoral experience. This session will last approximately one hour and I want you to be as honest as possible with your answers. I will keep all of the data confidential and I ask that you do not discuss the conversation today with anyone outside this room, to respect all participants.

1. Motivation

a. Tell me about why you decided to enroll in a $\mathrm{PhD}$ program.

b. When are you most motivated to complete the degree? When are you least motivated?

2. Satisfaction

a. How has your PhD experience been going?

b. Would you change anything about your $\mathrm{PhD}$ experience?

Thank you for taking the time to speak to me today. Later today, I will be sending each of you a link to an anonymous feedback form. This form will allow you to share any other experiences or details about your PhD experience. As a reminder, if you have any questions about the study, you can always contact me at heather.turner@louisville.edu.

This Qualtrics form will have one question with an accompanying text box:

1. Please use this form to add any details or information related to your personal motivation or your satisfaction with your $\mathrm{PhD}$ experience:

[LINK] 


\section{Appendix E}

\section{Relationship between Qualitative and Quantitative Research Strands}

\begin{tabular}{ll}
$\begin{array}{l}\text { Research Strand } \\
\text { Decision Points }\end{array}$ & \multicolumn{1}{c}{ Relationship to Study Design } \\
\hline Interaction & $\begin{array}{l}\text { This study adopted an interactive approach, as the qualitative } \\
\text { portion was refined based on the quantitative findings, and the } \\
\text { results from each strand were interpreted together to generate study } \\
\text { findings. }\end{array}$ \\
\hline Priority & $\begin{array}{l}\text { This study prioritized the quantitative strand as this portion of the } \\
\text { study will allow me to understand the relationship between } \\
\text { motivation and satisfaction for part-time PhD students and thus } \\
\text { build the foundation for further qualitative exploration. }\end{array}$ \\
\hline Timing & $\begin{array}{l}\text { This study followed sequential timing, in that the collection and } \\
\text { analysis of the strands were carried out separately. The study began } \\
\text { with the quantitative phase, as this strand maintained priority for } \\
\text { establishing the relationship between motivation and satisfaction. }\end{array}$ \\
\hline Procedures & $\begin{array}{l}\text { For this study, mixing strands occurred at the data collection stage, } \\
\text { as the results of one research strand inform the collection of the } \\
\text { data for the other research strand. Additionally, the study's design } \\
\text { falls on the continuum between fixed and emergent research } \\
\text { designs. }\end{array}$
\end{tabular}




\section{Appendix F}

\section{Coding Structure}

\begin{tabular}{|c|c|c|}
\hline \multicolumn{2}{|c|}{ Exploratory Coding } & First Cycle Code Examples \\
\hline \multirow[t]{7}{*}{$\begin{array}{l}\text { Autonomous } \\
\text { Motivations }\end{array}$} & \multirow[t]{3}{*}{$\begin{array}{r}\text { Reasons for pursuing the } \\
\text { PhD }\end{array}$} & $\begin{array}{l}\text { Being able to balance } \mathrm{PhD} \text { with } \\
\text { career/support family }\end{array}$ \\
\hline & & Wanting to address a problem \\
\hline & & Personal Goal \\
\hline & \multirow[t]{4}{*}{ Reasons for persisting } & Professional Knowledge \\
\hline & & Shared Experiences \\
\hline & & Goal oriented \\
\hline & & Role Modeling \\
\hline \multirow{10}{*}{$\begin{array}{r}\text { Controlled } \\
\text { Motivations }\end{array}$} & \multirow{4}{*}{$\begin{array}{l}\text { Reasons for pursuing the } \\
\qquad \mathrm{PhD}\end{array}$} & Need the credential \\
\hline & & Career Advancement \\
\hline & & Faculty/Advisor Encouragement \\
\hline & & Career Exploration \\
\hline & \multirow[t]{6}{*}{ Reasons for persisting } & Not wanting to let people down \\
\hline & & $\begin{array}{l}\text { Believing the PhD confers } \\
\text { authority and respect }\end{array}$ \\
\hline & & Career Advancement \\
\hline & & Sunk Costs \\
\hline & & Advisor Supports \\
\hline & & Tuition Remission \\
\hline \multirow{5}{*}{\multicolumn{2}{|c|}{ Demotivational Factors }} & Isolation \\
\hline & & Managing Competing Priorities \\
\hline & & Lack of Advisor Supports \\
\hline & & Exhaustion \\
\hline & & Impostor Syndrome \\
\hline \multirow{3}{*}{\multicolumn{2}{|c|}{ Contributors to Satisfaction }} & Advisor + \\
\hline & & Knowledge \\
\hline & & Social Support \\
\hline \multirow{6}{*}{\multicolumn{2}{|c|}{ Detractors from Satisfaction }} & Faculty prefer full-time students \\
\hline & & $\begin{array}{l}\text { Supports are not designed for part- } \\
\text { time students }\end{array}$ \\
\hline & & Program requirements are unclear \\
\hline & & COVID's impacts \\
\hline & & $\begin{array}{l}\text { Universities don't support part-time } \\
\text { students }\end{array}$ \\
\hline & & Lack of cohort support \\
\hline
\end{tabular}




\section{Appendix G}

\section{IRB Approval}

\section{U N I V ER S I T Y O F LOUISVILLE}

Human Subjects Protection Program Office

MedCenter One - Suite 200

501 E. Broadway

Louisville, KY 40202-1798

August 11, 2020

Meghan J Pifer, Ph.D.

TO:

FROM:

IRB NUMBER:

The University of Louisville Institutional Review Board

20.0583

STUDY TITL:

REFERENCE \#:

IRB STAFF

CONTACT:
An explanatory sequential mixed-methods study of part-time PhD students' motivation and satisaction

Jackie Powell, CIP 852-4101 jspowe01@louisille.edu

This study was reviewed and approved with changes on $08 / 04 / 2020$ by the Chair of the Institutional Review Board. The resubmitted changes were approved administratively on $08 / 11 / 2020$. This study was approved through Expedited Review Procedure, according to 45 CFR $46.110\{\mathrm{~b}\}$, since this study falls under Category 7: Research on individual or group characteristics or behavior (including, but not limited to, research on perception, cognition, motivation, identity, language, communication, cultural belief 5 or practices, and social behavior for research employing survey, interview, oral history, focus group, program evaluation, human factors evaluation, or quality assurance metho dologies

This study now has final IRB approval from 08/11/2020 through 08/10/2023.

This study was also approved through 45 CFR 46.116 \{C\}, which means that an IRB may waive the requirement for the irvestigator to obtain a signed informed consent form for some or all subjects.

The following items have been approved:

\begin{tabular}{|c|c|c|c|c|}
\hline \multicolumn{5}{|l|}{ Submission Components } \\
\hline Form Name & \multicolumn{2}{|l|}{ Version } & \multicolumn{2}{|l|}{ Outcome } \\
\hline Submit for Initial Review & \multicolumn{2}{|l|}{ Version 1.0} & \multicolumn{2}{|c|}{ Approved as Submitted } \\
\hline $\begin{array}{l}\text { Review Response Submission } \\
\text { Form }\end{array}$ & \multicolumn{2}{|l|}{ Version 2.0} & \multicolumn{2}{|c|}{ Approved as Submitted } \\
\hline IRB Study Application & \multicolumn{2}{|l|}{ Version 1.1} & \multicolumn{2}{|c|}{ Approved as Submitted } \\
\hline \multicolumn{5}{|l|}{ Study Document } \\
\hline \multicolumn{2}{|l|}{ Title } & Version \# & Version Date & Outcome \\
\hline \multicolumn{2}{|l|}{ Email script - Clean } & Version 2.0 & $08 / 05 / 2020$ & Approved \\
\hline \multicolumn{2}{|l|}{ Instrument - Clean } & Version 2.0 & $08 / 05 / 2020$ & Approved \\
\hline \multicolumn{2}{|l|}{ Protocol - Clean } & Version 2.0 & $08 / 05 / 2020$ & Approved \\
\hline \multicolumn{2}{|c|}{ Qualitative instrument Focus Group Protocol } & Version 1.0 & $07 / 16 / 2020$ & Approved \\
\hline \multicolumn{2}{|c|}{ Protocol - Dissertation proposal } & Version 1.0 & $07 / 10 / 2020$ & Approved \\
\hline \multicolumn{2}{|l|}{ Unsigned Consent_Focus Group } & Version 2.0 & $08 / 05 / 2020$ & Approved \\
\hline
\end{tabular}

IRB policy requires that investigators use the IRB "stamped" approved version of informed consents, 
assents, and other materialsgiven to research participants. For instructions on locating the IRB stamped documents in iRIS visit: https://ouisville.edu/research/humansubjects/iRISSubmissionManual.pdf

Your study does not require annual continuing review. Your study has been set with a three year expiration date. If your study is still ongoing you will receive iRIS auto mated reminders to submit a request to continue your study prior to the expiration date above.

All other IRB requirements are still applicable. You are still required to submit amendments, personnel changes, deviations, etc... to the IRB for review. Please submit a closure amendment to close out your study with the IRB if it ends prior to the three year expiration date.

Human subjects \& HIPAA Research training are required for all study personnel. It is the responsibility of the investigator to ensure that all study personnel maintain current Human Subjects \& HIPAA Research training while the study is ongoing.

\section{Site Approval}

Permission from the institution or organization where this research will be conducted must be obtained before the research can begin. For example, site approval is required for research conducted in UofL Hospital/UofL Health, Norton Healthcare, and J efferson County Public Schools, etc...

\section{Privacy \& Encryption Statement}

The University of Louisville's Privacy and Encryption Policy requires identifiable medical and health records; credit card, bank account and other personal financial information; social security numbers; proprietary research data; and dates of birth (when combined with name, address and/or phone numbers\} to be encrypted. For additional information: http://ouisville.edu/security/policies.

\section{Implementation of Changes to Previously Approwed Research}

Prior to the implementation of any changes in the approved research, the irwestigato r must submit modifications to the IRB and await approval before implementing the changes, unless the change is being made to ensure the safety and welfare of the subjects enrolled in the research. If such occurs a Protocol Deviation/violation should be submitted within five days of the occurrence indicating what safety measures were taken, along with an amendment to revise the protocol.

\section{Unanticipated Problems Involying Risks to Subjects or Others (UPIRTSOs)}

A UPIRTSO is any incident, experience, or outcome, which has been associated with an unexpected event \{s\}, related or possibly related to participation in the research, and suggests that the research places subjects or others at agreater risk of harm than was previous y known or suspected. The investigator is respons ble for reporting UPIRTSOs to the IRB within 5 working days. Use the UPIRTSO form located within the iRIS system. Event reporting requirements can be found at:

http://ouisille.edu/research/humansubjects/lifecycle/event-reporting.

\section{Payments to Subjects}

Full Accreditation since June 2005 by the Association for the Accreditation of Human Research Protection Frograms, Inc.

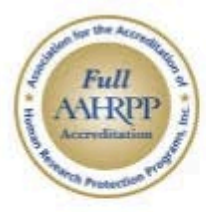


In compliance with University policies and Internal Revenue Service code, payments to research subjects from University of Louisville funds, must be reported to the University Controller's Office. For additional information, please call 852-8237 or email controll@louisville.edu. For additional information: http://louisville.edu/research/humansubjects/policies/PayingHumanSubjectsPolicy201412.pdf

The committee will be advised of this action at a regularly scheduled meeting.

If you have any questions, please contact: Jackie Powell 852-4101 jspowe01@louisville.edu

\section{Pate $m$ Lusach}

Peter M. Quesada, Ph.D., Chair

Social/Behavioral/Educational Institutional Review Board

\section{$\mathrm{PMQ} / \mathrm{jsp}$}

We value your feedback; let us know how we are doing: https://www.surveymonkey.com/r/CCLHXRP

Full Accreditation since June 2005 by the Association for the Accreditation of

Human Research Protection Programs, Inc

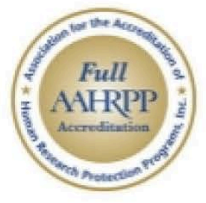




\title{
CURRICULUM VITAE
}

\author{
Heather A. Turner
}

\section{Education}

Ph.D. (expected 2021) | University of Louisville, Educational Leadership and

Organizational Development

M.A. | University of Louisville, Higher Education Administration

M.A. University of Delaware, English, Concentration in Rhetoric and Composition

B.A. | Temple University, English, Summa Cum Laude

Administration

Director of Research and Policy | 2020 - Present

University of Louisville, Louisville, Kentucky.

Program Manager for the Master Educator Course | 2017 - 2020

University of Louisville, Louisville, Kentucky.

Assistant to the Director of the Office of National and International Scholarships | 2014 2016 University of Louisville, Louisville, Kentucky.

Assistant Director of the Writing Center | 2013 - 2014

University of Delaware. Newark, Delaware.

\section{Teaching}

Instructor | 2012 - 2017

University of Louisville, Louisville, Kentucky.

- ENGL101: Introduction to College Writing

- ENGL102: Intermediate College Writing

- ENGL102 (Distance Education): Intermediate College Writing

University of Delaware, Newark, Delaware.

- ENGL110: Critical Reading and Writing.

- ENGL 392: Teaching Writing One-to-One.

\section{Advising}

Online Enrollment Counselor | 2016 - 2017

University of Louisville, Louisville, Kentucky.

Interim Honors Advisor | 2015

University of Louisville, Louisville, Kentucky.

\section{Editorial and Review}

First Round Scholarship Reviewer | 2014 - 2016

Critical Language Scholarship, Washington D.C.

Scholarship Application Reviewer | 2015

Trustees Scholarship, University of Louisville, Louisville, Kentucky 
Assistant Editor | 2013

Writing Center Journal, Newark, Delaware.

Proposal Reviewer | 2013

Mid-Atlantic Writing Center Association, Newark, Delaware

\section{Publications}

Buckley, J., Turner, H., Cook, R. \& Pifer, M. (2020). It's not in the syllabus!: Selfauthorship and transitions for military connected students. In J. Garvey et al. Case Studies for Student Development Theory: Advancing Social Justice \& Inclusion in Higher Education. London, England: Routledge

Buckley, J., Turner H. (Under Review) When hallowed halls become firewalls: An integrative literature review informing an inclusive model of engaging nontraditional postsecondary students online

\section{Peer-Reviewed Conference Papers \& Presentations}

Turner, H. (2018, April) Ethical education for military-connected students: History, theory, and practice. Paper presented at the joint research conference of the University of Louisville, the University of Kentucky, and the University of Cincinnati, Louisville, KY.

Buckley, J. \& Turner, H. (2019, November) When hallowed halls become firewalls: An integrative literature review informing an inclusive model of engaging nontraditional postsecondary students online. Paper presented at the Annual Conference of the Association of the Study of Higher Education, Portland, OR

Pifer, M., Sun, J., \& Turner, H. (2019, November) ROTC as campus environments: Reimagining opportunities for learning partnerships on college and university campuses. Paper presented at the Annual Conference of the Association of the Study of Higher Education, Portland, OR

Turner, H. \& Pratt, T. (2021, February) Speaking their language: Translation and military support services in a virtual environment. Paper presented at the National Association of Student Personnel Administrators Symposium on MilitaryConnected Students, Virtual

Pifer, M., Sun, J., Pratt, T., \& Turner, H. (2021, April) A part, but apart: An exploration of legitimacy-seeking goals, behaviors, and outcomes within ROTC. Paper to be presented at the Annual Conference of the American Educational Research Association, Virtual

\section{Award and Fellowship}

Graduate Student Policy Seminar Fellow | 2019

Association for the Study of Higher Education, Portland, OR

Faculty Favorite Nominee | 2018

University of Louisville, Louisville, Kentucky 